\title{
Direct Writing of Aluminum Doped Zinc Oxide for Optoelectronic and Energy Device Applications
}

Olatunde Ayodeji Abidakun

Follow this and additional works at: https://researchrepository.wvu.edu/etd

\section{Recommended Citation}

Abidakun, Olatunde Ayodeji, "Direct Writing of Aluminum Doped Zinc Oxide for Optoelectronic and Energy Device Applications" (2015). Graduate Theses, Dissertations, and Problem Reports. 5020.

https://researchrepository.wvu.edu/etd/5020

This Thesis is protected by copyright and/or related rights. It has been brought to you by the The Research Repository @ WVU with permission from the rights-holder(s). You are free to use this Thesis in any way that is permitted by the copyright and related rights legislation that applies to your use. For other uses you must obtain permission from the rights-holder(s) directly, unless additional rights are indicated by a Creative Commons license in the record and/ or on the work itself. This Thesis has been accepted for inclusion in WVU Graduate Theses, Dissertations, and Problem Reports collection by an authorized administrator of The Research Repository @ WVU. For more information, please contact researchrepository@mail.wvu.edu. 


\title{
Direct Writing of Aluminum Doped Zinc Oxide for Optoelectronic and Energy Device Applications
}

\section{Olatunde Ayodeji Abidakun}

\author{
Thesis submitted to the \\ Benjamin M. Statler College of Engineering and Mineral Resources \\ At West Virginia University
}

In partial fulfillment of the requirements for the degree of

Master of Science in Mechanical Engineering

Konstantinos Sierros, Ph.D., Chair

Samir Shoukry, Ph.D.

Charter Stinespring, Ph.D.

Department of Mechanical and Aerospace Engineering

Morgantown, West Virginia

2015

Keywords: Direct writing - Zinc Oxide - Aluminum - X-Ray Diffraction - Microstructure Copyright 2015 Olatunde Ayodeji Abidakun 


\section{ABSTRACT \\ Direct Writing of Aluminum Doped Zinc Oxide for Optoelectronic and Energy Device Applications \\ Olatunde Ayodeji Abidakun}

Micro scale patterning of transparent conducting oxide (TCO) offers great promise in producing efficient and low cost devices. Most of the major patterning techniques are either complex, expensive, non-scalable, or involve the use of environmentally unfriendly chemicals. Therefore, patterning of Aluminum doped Zinc Oxide (AZO) via sol-gel route, combined with direct writing, in particular, nozzle-based robotic deposition, offers a relatively easy and efficient way to selectively deposit complex features in digitally precise locations, at low cost and without excessive use of harmful chemicals. However, the printing process involves the interplay of various parameters, which need to be properly related, in order to annex the inherent benefits in the manufacturing process.

Hence, the focus of this work is to investigate the influence of the printing parameters, which are viscosity, nozzle size, extrusion pressure, dispensing height and writing speed on the properties of the patterned AZO feature on glass substrates. The properties of interest include geometry of deposited and sintered feature, morphology, microstructure and electrical conductivity. A dual, three factors, three levels factorial experiment was designed to determine the influence of the printing parameters on the feature geometry. The morphology and crystal structure of the sintered feature at various combinations of printing parameters were studied by scanning electron microscopy (SEM) and x-ray diffraction (XRD). Also, the electrical properties of sintered feature with and without ultraviolet (UV) light irradiation were determined in a metalsemiconductor-metal configuration using a semiconductor analyzer.

It was found that the main effects and interactions among the printing parameters considered were significant in determining the spread and the profile of the deposited features at 95\% confidence level. A link between the printing parameters and the morphology and crystal structure was established, providing the opportunity for tailoring the deposited film to desired end use, without altering the chemical composition or post processing treatment. The electrical property, which increased upon UV excitation, was also found to be comparable with those reported in the literature for conventionally sol-gel deposited AZO films.

Given the achieved control of feature geometry and microstructure through alteration of printing parameters, and the electrical conductivity of the deposited patterns, this work provides a pathway to efficient, large area optoelectronic devices at low cost. 


\section{ACKNOWLEDGEMENTS}

I would like to express my sincere gratitude to my advisor, Dr. Kostas Sierros for his guidance and support through course of this program, and for always being committed to helping me succeed. His in-depth knowledge of the subject field has help me grow professionally.

My sincere appreciation goes to Dr. Samir Shoukry and Dr. Charter Stinespring for agreeing to serve in my committee. Their relentless support and timely response is highly appreciated.

I thank my colleagues Maria Torres, Derrick Banerjee, Jacob Cordonier and all the undergraduates in the FEST group for their support and contributions during the course of this research. I also thank Sean Cronin, Nick Morris, Sai Ramayanam, and Mark Shoukry for their assistance.

Finally, I express my sincere gratitude to my wife Esther Abidakun, my daughter Omotola Abidakun, my parents Michael Abidakun, Julianah Abidakun, my inlaws Samuel Akano, Mary Akano, my siblings Oluwakemi Oni, Akinbayo Abidakun, Olufisayo Abidakun, Olubusayo Abidakun, for their unconditional love, sacrifices, support and encouragement during this programme. Your contributions in making this thesis a success are immensurable. 


\section{TABLE OF CONTENTS}

Acknowledgements. ............................................................................................................ii

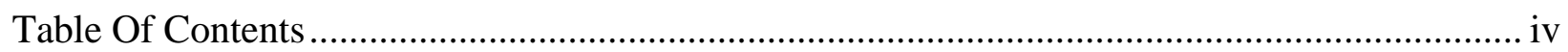

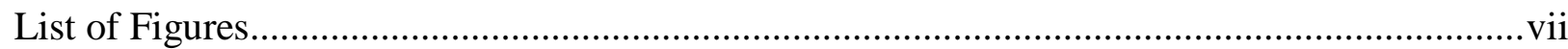

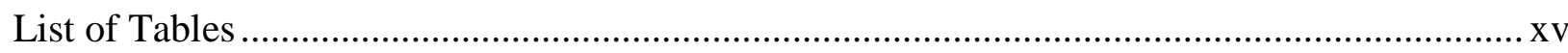

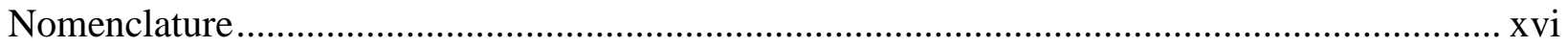

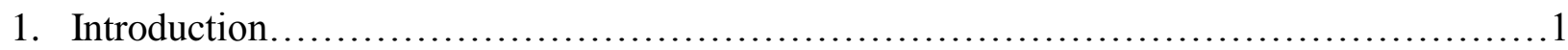

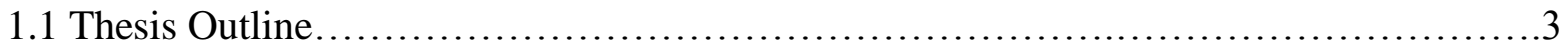

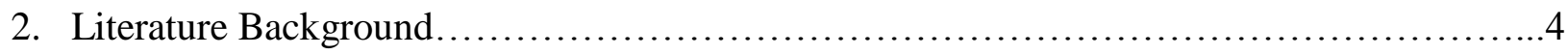

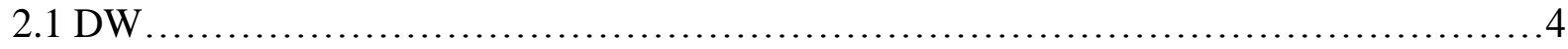

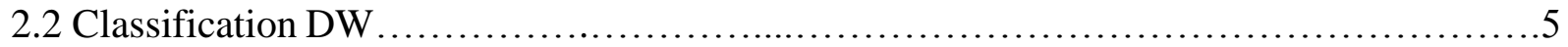

2.3 Applications of DW Techniques in Device Manufacturing ............................... 8

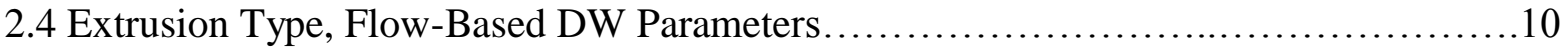

2.5 Structure, Properties and Applications of Zinc oxide $(\mathrm{ZnO})$ Thin Film....................11

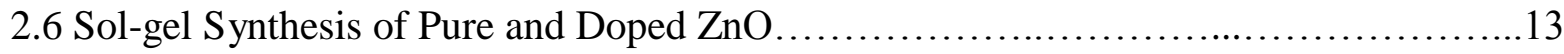

2.7 ZnO Thin Film Deposition and Characterization Methods...............................15

2.8 Post Processing of Deposited ZnO Thin Films......................................... 18

2.9 ZnO Thin Film Electrical Conductivity and UV Detection............................19

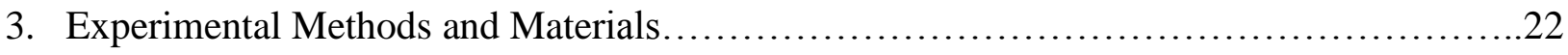

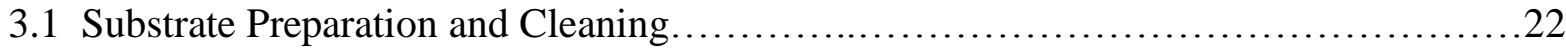

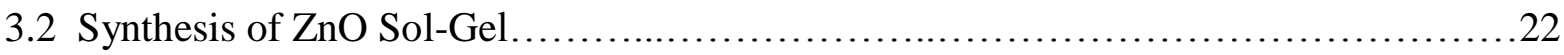

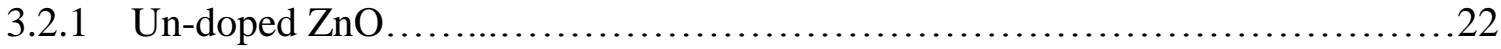

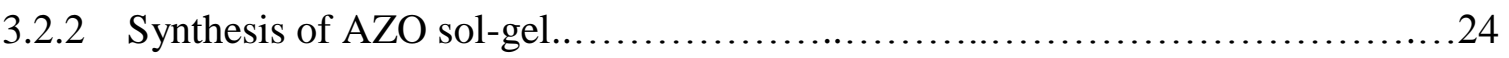




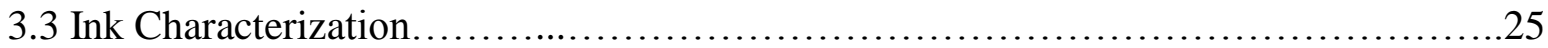

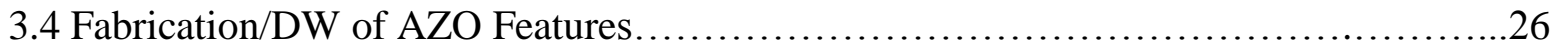

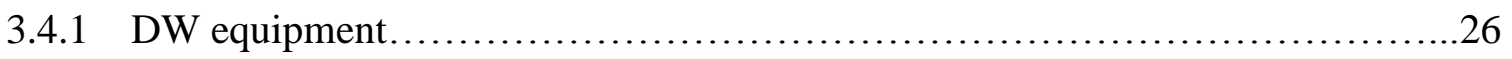

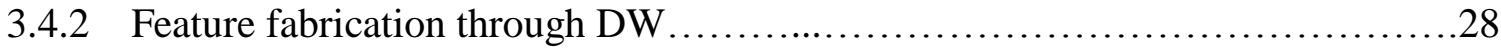

3.5 Interaction and Significance of the Printing Parameters...............................31

3.6 Drying and Sintering of Printed Patterns............................................ 33

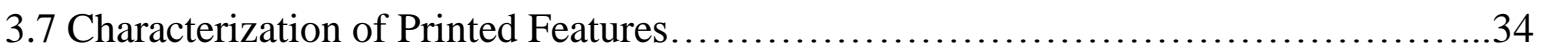

3.7.1 Geometrical characterization.......................................... 34

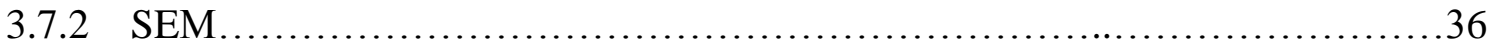

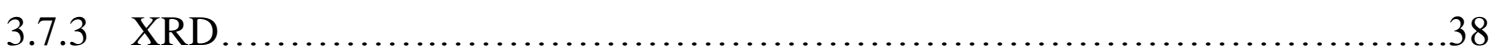

3.7.4 Electrical characterization..............................................41

4. DW of AZO Inks: Investigation of Printing Parameters.................................43

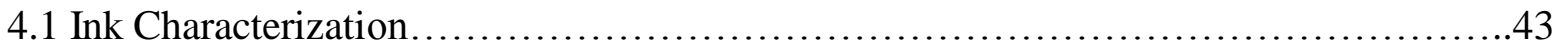

4.2 Effect of Printing Parameters...................................................48

4.3 Significance and Interactions of Printing Parameters.................................64

4.4 Printing Mechanism with the Extrusion Type Flow- Based DW ......................69

4.4 Effect of Post Deposition Treatment on Feature Geometry..............................71

5. Effect of Printing Parameters on Resulting Microstructure of Written Pattern..................76

5.1 Crystal Structure and Morphology ................................................ 76

5.2 Dependence of Micro Structure on Printing Parameters............................ 82

5.3 Crystal Growth Mechanism................................................... 88 
6. Electrical Characterization of Directly Written Patterns................................95

6.1 Effect of Printing Conditions on Film Conductivity............................95

6.2 Photoconductivity of Printed AZO Films ....................................98

7. Conclusions and Recommendations for Future Work ............................... 103

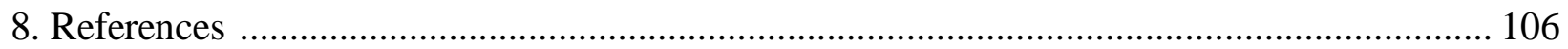




\section{LIST OF FIGURES}

Figure 2.1 Pictorial representation of direct writing comparison with other electronic fabrication methods .6

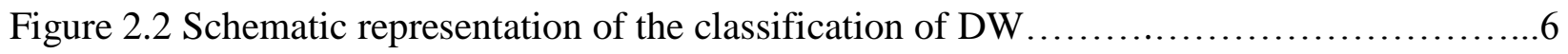

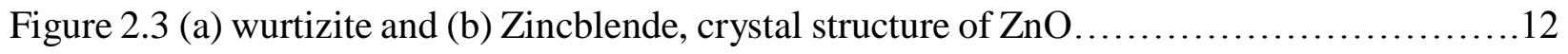

Figure 3.1: Schematic of the un-doped $\mathrm{ZnO}$ preparation process.........................23

Figure 3.2: Schematic representation of the AZO synthesis...............................25

Figure 3.3: Images of the (a) Nordson Multi-axis robot and (b) Nordson Performus V pressure

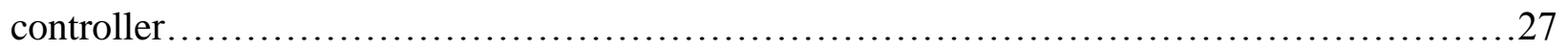

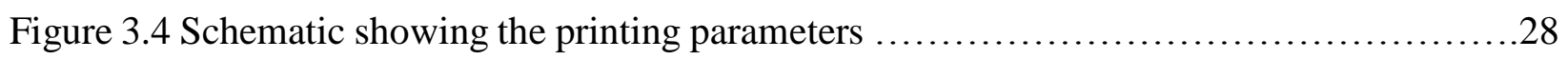

Figure 3.5 Schematic representation of the feature printing process........................30

Figure 3.6 (a) Optical images of a feature printed with $100 \mu \mathrm{m}$ tip, 20 psi pressure, $100 \mu \mathrm{m}$ dispensing height and $3 \mathrm{~mm} / \mathrm{s}$ writing speed using ink 2 and (b) digital image of a $100 \mu \mathrm{m}$ tip used

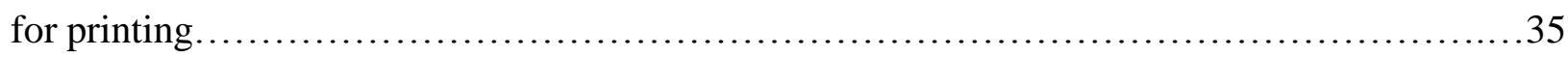

Figure 3.7 Schematic representation of a printed feature's cross sectional view................36

Figure 3.8 Schematics showing SEM operating principle............................... 37

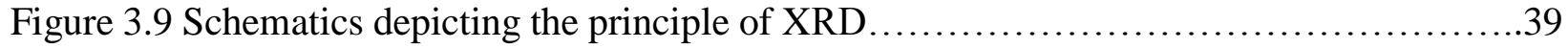

Figure 3.10 Schematic of the MSM device..........................................41

Figure 3.11 Schematic representation of the electrical characterization process................42 
Figure 4.1 Viscosity of the three inks versus shear rate.

Figure 4.2 Change in viscosity for inks with different PVP contents.

Figure 4.3 Viscosity of ink $1(10 \mathrm{wt} \% \mathrm{PVP})$ at different shear rates. .45

Figure 4.4 Viscosity of ink 2 (15 wt\% PVP) at different shear rate. .46

Figure 4.5 Viscosity of ink 3 (20 wt $\%$ PVP) at different shear rate.

Figure 4.6 Contact angle measurement of AZO inks with different PVP contents.

Figure 4.7 Contact angle of deionized water and the three AZO inks on the cleaned glass substrates' surface. .47

Figure 4.8 Typical image of patterns drawn at high dispensing height

Figure 4.9 Spread for feature printed with different inks and pressures at $1 \mathrm{~mm} / \mathrm{s}$ writing speed, using $150 \mu \mathrm{m}$ nozzle and $150 \mu \mathrm{m}$ dispensing height. .51

Figure 4.10 Spread for feature printed with different inks and pressures at $5 \mathrm{~mm} / \mathrm{s}$ writing speed, using $150 \mu \mathrm{m}$ nozzle and $150 \mu \mathrm{m}$ dispensing height. .51

Figure 4.11 Spread for feature printed with different inks and writing speeds at 15 psi, using 150 $\mu \mathrm{m}$ nozzle and $150 \mu \mathrm{m}$ dispensing height. .52

Figure 4.12 Spread for feature printed with different tip sizes and pressures at $1 \mathrm{~mm} / \mathrm{s}$ writing speed, using ink 2 (15 wt\% PVP) and same dispensing heights as the tip sizes.... .53

Figure 4.13 Spread for feature printed with different tip sizes and pressures at $5 \mathrm{~mm} / \mathrm{s}$ writing speed, using ink 2 (15 wt\% PVP) and same dispensing heights as the tip sizes...... .53 
Figure 4.14 Spread for feature printed with different tip sizes and writing speeds at 15 psi, using ink $2(15 \mathrm{wt} \%$ PVP) and same dispensing heights as the tip sizes........................54

Figure 4.15 Spread versus pressure for features printed with different inks at $1 \mathrm{~mm} / \mathrm{s}$ using $150 \mu \mathrm{m}$

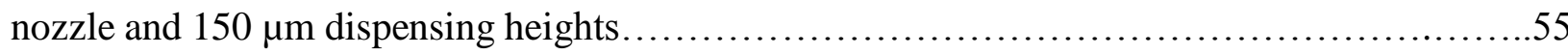

Figure 4.16 Spread versus pressure for features printed with different inks at $1 \mathrm{~mm} / \mathrm{s}$ using 150 $\mu \mathrm{m}$ nozzle and $150 \mu \mathrm{m}$ dispensing heights.

Figure 4.17 Spread versus pressure for features printed with different inks at $1 \mathrm{~mm} / \mathrm{s}$ using 150

$\mu \mathrm{m}$ nozzle and $150 \mu \mathrm{m}$ dispensing heights........................................ 56

Figure 4.18 Spread versus pressure for features printed with different tip sizes at $1 \mathrm{~mm} / \mathrm{s}$ using ink 2 and same dispensing heights as the tip sizes.

Figure 4.19 Spread versus pressure for features printed with different tip sizes at $5 \mathrm{~mm} / \mathrm{s}$ using ink 2 and same dispensing heights as the tip sizes. .57

Figure 4.20 Spread versus writing speed for features printed with different inks at 10 psi using $150 \mu \mathrm{m}$ tip size and dispensing height. .59

Figure 4.21 Spread versus writing speed for features printed with different inks at 15 psi using $150 \mu \mathrm{m}$ tip size and dispensing height.

Figure 4.22 Spread versus writing speed for features printed with different tip sizes at 10 psi using ink 2 and same dispensing heights as the tip sizes.

Figure 4.23 Spread versus writing speed for features printed with different tip sizes at 20 psi using ink 2 and same dispensing heights as the tip sizes. 
4.24 Profile of feature written with ink 2, $100 \mu \mathrm{m}$ tip size and 10 psi at different writing speeds.

62

4.25 Profile of feature written with ink 2, $100 \mu \mathrm{m}$ tip size and 15 psi at different writing speeds.

4.26 Profile of feature written with ink 3, $150 \mu \mathrm{m}$ tip size and 10 psi at different writing speeds.

4.27 Profile of feature written with ink $2,100 \mu \mathrm{m}$ tip size and 15 psi at different writing speeds..63

Figure 4.28 Order of importance of printing parameters

Figure 4.29 Feature width versus writing speed and volumetric flow rate. .68

Figure 4.30 Schematic representation of the printing process and mechanism .70

Figure 4.31 TGA of the inks used for printing .71

Figure 4.32 Before and after sintering spreads versus writing speed for feature printed with ink 2, $150 \mu \mathrm{m}$ nozzle size and 15 psi pressure .72

Figure 4.33 Before and after sintering spreads versus writing speed for feature printed with ink 2, $200 \mu \mathrm{m}$ nozzle size and 20 psi pressure .73

Figure 4.34 Before and after sintering spreads for inks with different viscosities printed with ink $150 \mu \mathrm{m}$ nozzle size, $10 \mathrm{psi}$ and $3 \mathrm{~mm} / \mathrm{s}$ speed.

Figure 4.35 Before and after sintering spreads for inks with different viscosities printed with ink $150 \mu \mathrm{m}$ nozzle size, $20 \mathrm{psi}$ and $3 \mathrm{~mm} / \mathrm{s}$ speed. .74 
Figure 4.36 Before and after sintering profile for feature printed with ink 2, $150 \mu \mathrm{m}$ nozzle size,

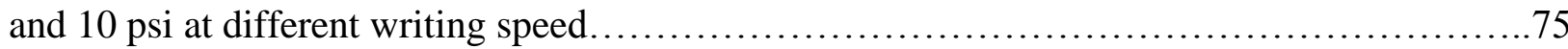

Figure 4.37 Before and after sintering profile for feature printed with ink 2, $150 \mu \mathrm{m}$ nozzle size,

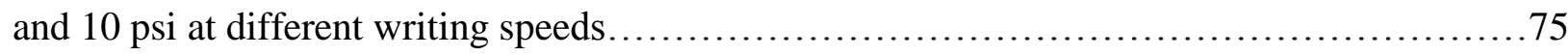

Figure 5.1 XRD spectra for AZO films deposited with different printing parameters............79

Figure 5.2 SEM images of feature printed with ink 2, $100 \mu \mathrm{m}$ nozzle, $15 \mathrm{psi}$ and $3 \mathrm{~mm} / \mathrm{s}$, and

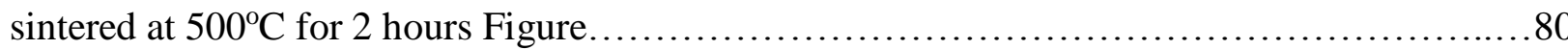

5.3 SEM images of feature printed with ink 2, $150 \mu \mathrm{m}$ nozzle, $15 \mathrm{psi}$ and $3 \mathrm{~mm} / \mathrm{s}$, and sintered

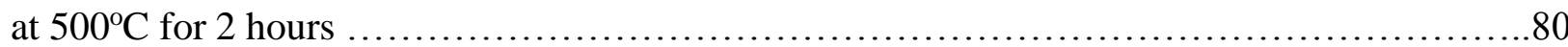

Figure 5.4 SEM images of feature printed with ink 2, $150 \mu \mathrm{m}$ nozzle, $15 \mathrm{psi}$ and $1 \mathrm{~mm} / \mathrm{s}$, and sintered at $500^{\circ} \mathrm{C}$ for 2 hours.

Figure 5.5 SEM images of feature printed with ink 2, $200 \mu \mathrm{m}$ nozzle, $15 \mathrm{psi}$ and $3 \mathrm{~mm} / \mathrm{s}$, and sintered at $500^{\circ} \mathrm{C}$ for 2 hours

Figure 5.6 XRD spectrum of feature printed with ink 2, $100 \mu \mathrm{m}$ nozzle, 15 psi at speeds of 1 and $3 \mathrm{~mm} / \mathrm{s}$, and sintered at $500^{\circ} \mathrm{C}$ for 2 hours.

Figure 5.7 XRD spectrum of feature printed with ink 2, $100 \mu \mathrm{m}$ nozzle, 20 psi at speeds of 3 and $5 \mathrm{~mm} / \mathrm{s}$, and sintered at $500^{\circ} \mathrm{C}$ for 2 hours.

Figure 5.8 XRD spectrum of feature printed with ink 2, $150 \mu \mathrm{m}$ nozzle, $3 \mathrm{~mm} / \mathrm{s}$ and 10, 15 and 20 psi pressure, and sintered at $500^{\circ} \mathrm{C}$ for 2 hours

Figure 5.9 XRD spectrum of feature printed with ink 2, $150 \mu \mathrm{m}$ nozzle, $15 \mathrm{psi}$ and 1 and $3 \mathrm{~mm} / \mathrm{s}$, and sintered at $500^{\circ} \mathrm{C}$ for 2 hours. 
Figure 5.10 XRD spectrum of feature printed with ink 2, $200 \mu \mathrm{m}$ nozzle, 15 psi and 1, 3 and 5 $\mathrm{mm} / \mathrm{s}$, and sintered at $500^{\circ} \mathrm{C}$ for 2 hours .85

Figure $5.11 \mathrm{XRD}$ spectrum of feature printed with ink 2, $100 \mu \mathrm{m}$ nozzle, 20 psi at 3 and $5 \mathrm{~mm} / \mathrm{s}$, and sintered at $500^{\circ} \mathrm{C}$ for 2 hours

Figure 5.12 XRD spectrum of feature printed with ink 2, $100 \mu \mathrm{m}$ nozzle, $15 \mathrm{psi}$ at 1 and $3 \mathrm{~mm} / \mathrm{s}$, and sintered at $500^{\circ} \mathrm{C}$ for 2 hours .88

Figure 5.13 XRD spectrum of feature printed with ink 2, $150 \mu \mathrm{m}$ nozzle, $15 \mathrm{psi}$ at 1 and $3 \mathrm{~mm} / \mathrm{s}$, and sintered at $500^{\circ} \mathrm{C}$ for 2 hours .88

Figure 5.14 XRD spectrum of feature printed with ink 2, $200 \mu \mathrm{m}$ nozzle, $3 \mathrm{~mm} / \mathrm{s}$ at 10, 15 and 20 psi, and sintered at $500^{\circ} \mathrm{C}$ for 2 hours.

Figure 5.15 Schematic of the observed crystal orientation at different feature widths .89

Figure 5.15 XRD spectrum of feature printed with ink 3, $150 \mu \mathrm{m}$ nozzle, $3 \mathrm{~mm} / \mathrm{s}$ at 10, 15 and 20 psi, and sintered at $500^{\circ} \mathrm{C}$ for 2 hours .92

Figure 5.16 XRD spectrum of feature printed with ink 1, $150 \mu \mathrm{m}$ nozzle, $3 \mathrm{~mm} / \mathrm{s}$ at 10, 15 and 20 psi, and sintered at $500^{\circ} \mathrm{C}$ for 2 hours .90

Figure 5.17 XRD spectrum of feature printed with ink 2, $200 \mu \mathrm{m}$ nozzle, $3 \mathrm{~mm} / \mathrm{s}$ at 10, 15 and 20 psi, and sintered at $500^{\circ} \mathrm{C}$ for 2 hours 91

Figure 5.18 XRD spectrum of feature printed with ink 1, $150 \mu \mathrm{m}$ nozzle, $3 \mathrm{~mm} / \mathrm{s}$ at 10, 15 and 20 psi, and sintered at $500^{\circ} \mathrm{C}$ for 2 hours

Figure 5.19 XRD spectrum of feature printed with ink 2, $200 \mu \mathrm{m}$ nozzle, $3 \mathrm{~mm} / \mathrm{s}$ at 10, 15 and 20 psi, and sintered at $500^{\circ} \mathrm{C}$ for 2 hours. 
Figure 5.20 SEM image for ink 2 printed with $100 \mu \mathrm{m}$ nozzle, $15 \mathrm{psi}$ and $3 \mathrm{~mm} / \mathrm{s}$, and sintered at $500^{\circ} \mathrm{C}$ for 2 hours .93

Figure 5.21 SEM image for ink 2 printed with $100 \mu \mathrm{m}$ nozzle, $15 \mathrm{psi}$ and $3 \mathrm{~mm} / \mathrm{s}$, and sintered at $500^{\circ} \mathrm{C}$ for 2 hours.

Figure 5.22 Schematic representation of perceived crystal nucleation and growth mechanism.....94

Figure 6.1 Current versus voltage for pattern printed with ink 1, $150 \mu \mathrm{m}$ nozzle and $10 \mathrm{psi}$ pressure at different speeds. .96

Figure 6.2 Current versus voltage for pattern printed with ink 2, $150 \mu \mathrm{m}$ nozzle and $20 \mathrm{psi}$ pressure at different speeds. .. 97

Figure 6.3 Current versus voltage for pattern printed with ink 3, $150 \mu \mathrm{m}$ nozzle and $15 \mathrm{psi}$ pressure at different speeds. .97

Figure 6.4 Current versus voltage for pattern printed with ink 1, $150 \mu \mathrm{m}$ nozzle, 10 psi and 3 $\mathrm{mm} / \mathrm{s}$, with and without UV excitation....

Figure 6.5 Current versus voltage for pattern printed with ink 2, $150 \mu \mathrm{m}$ nozzle, 10 psi and 3 $\mathrm{mm} / \mathrm{s}$, with and without UV excitation.

Figure 6.6 Resistance versus temperature for dark and UV irradiated films ....

Figure 6.7 Transient resistance of AZO film deposited with ink 2, $15 \mathrm{psi}$ and $3 \mathrm{~mm} / \mathrm{s}$ at $25^{\circ} \mathrm{C}$

Figure 6.8 Transient resistance of AZO film deposited with ink 2, $15 \mathrm{psi}$ and $3 \mathrm{~mm} / \mathrm{s}$ at $75^{\circ} \mathrm{C}$ 
Figure 6.9 Transient resistance of AZO film deposited with ink 2, $15 \mathrm{psi}$ and $3 \mathrm{~mm} / \mathrm{s}$ at

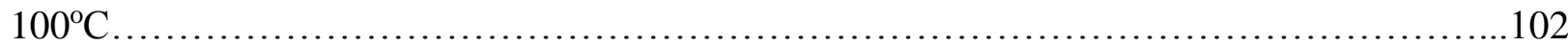




\section{LIST OF TABLES}

Table 3.1: Composition of different un-doped $\mathrm{ZnO}$ inks used in this study.................23

Table 3.2 Factors, levels and the degree of freedoms of experimental parameters..............32

Table 4.1 Analysis of variance (ANOVA) for feature width based on viscosity, writing speed and

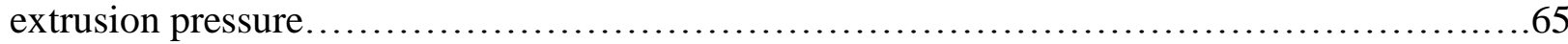

Table 4.2 Analysis of variance (ANOVA) for feature width based on tip size, writing speed and

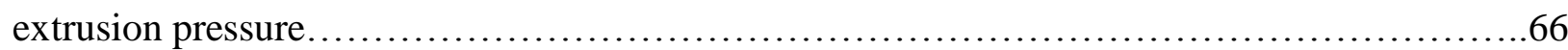

Table 5.1 XRD parameters along 002 peak for AZO films printed with different printing

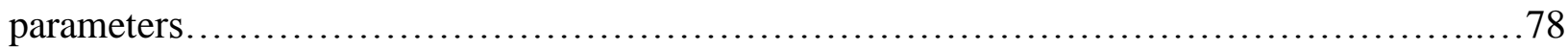




\section{NOMENCLATURE}

AZO: Aluminum doped Zinc Oxide

BHJ: Bulk Heterojunction

DPN: Dip Pen Nanolithography

DTI: Department of Trade and Industry

DW: Direct Writing

NBDW: Nozzle Based Direct Writing

NBRD: Nozzle Based Robotic Deposition

OFET: Organic Field Effect Transistor

PL: Photoluminescence

PVP: Polyvinyl Pyrolidone

SEM: Scanning Electron Microscopy

TCO: Transparent Conducting Oxide

TFT: Thin Film Transistor

UV: Ultra Violet

UV-VIS-NIR: Ultra Violet-Visible-Near Infrared

XRD: X-Ray Diffraction

ZnO: Zinc Oxide 


\section{INTRODUCTION}

$\mathrm{ZnO}$ based semiconductor patterned on conductive and insulating substrates has found wide applications in the areas of optoelectronics, photonics, microelectronics and energy devices. These application areas are traceable to ZnO's unique properties, which include a wide direct band gap of $3.37 \mathrm{eV}$, a high excitation binding energy of $60 \mathrm{meV}$, and a high optical transmission in the visible range [1]. Patterning of $\mathrm{ZnO}$ and related materials are currently being studied through various depositions methods, which include physical and chemical vapor deposition [2] [3], electroless plating [4], lithographic methods [5], spray pyrolysis [6], magnetron sputtering [7], spin coating [8] as well as direct writing deposition such as inkjet printing [9], dip pen nanolithography (DPN) [10], and electrospinning [11]. These methods are typically capable of depositing high purity materials with controlled thickness and surface morphology.

However, most of these major and established methods are complex, expensive, nonscalable for high volume production or are confined to deposition in small areas. For instance, vapor deposition methods are vacuum based and expensive with the need for post process lithography in order to create the desired functional patterns. Inkjet printing is limited to low viscosity functional inks preventing patterning in complex three dimensional architecture. Furthermore, DPN is limited to a small deposition area and electrospinning does not enable efficient control of the deposited patterns. These shortcomings have, however, contributed to the hindrances encountered in the production of low cost optoelectronic and energy devices.

There is therefore, the need for a deposition method that is relatively easy to execute, less

costly, capable of depositing over a large area in a controlled manner and scalable for high volume production in a roll-to-roll configuration. Nozzle based robotic deposition (NBRD), a type of extrusion based, flow type direct writing (DW), can potentially meet such requirements. NBRD is 
capable of controlled deposition of functional features in precise digital locations using inexpensive and disposable components. Its operation is based on continuous extrusion/flow of tuned viscoelastic inks from a tube container and has the capability to accommodate materials over a wide range of viscosities [12] [13] [14].

Effective application of this method for efficient printing requires the appropriate matching of ink material properties (i.e. rheological properties) and printing parameters. The printing parameters often considered in the use of this deposition technique include the pressure, which is used for extruding materials from the nozzle, the size of the channel through which the material is expected to flow, the gap between the nozzle tip and the substrate surface, and the speed at which the nozzle traverses the surface [15]. It is crucial to properly combine these parameters, as they usually determine the resulting properties of the deposited features such as geometry, morphology, and microstructure [16].

In this work, we study the effects of different combinations of material and printing parameters on the properties of as deposited and sintered AZO features. The widths and profiles of deposited AZO films printed at different conditions are obtained with a view to gaining an insight into the dynamics of the interactions among the printing parameters. Impact of sintering on the deposited patterns are also evaluated, for the purpose of understanding the drying, decomposition and crystallization behavior of the films.

We also report on the morphology, microstructure and crystal orientations of film deposited at various printing conditions, as determined by the use of SEM and XRD. Lastly, the electrical properties of deposited and sintered film with and without UV irradiation is also examined. 


\subsection{Thesis Outline}

The remaining part of this thesis is organized as follows: Chapter 2 discusses the nature, classification, advantages, and applications of direct writing technique. Structure, properties and applications of $\mathrm{ZnO}$ thin films are also discussed. Discussions about the various researches on the synthesis, deposition and characterization of doped and undoped $\mathrm{ZnO}$ thin films are also included.

Chapter 3 presents the methodology used in the research including AZO ink synthesis, printing with NBRD and resulting characterization techniques.

Chapter 4 presents and discusses the effect of different combinations of material and printing parameters on the geometry of the deposited film. It also shows the effect of sintering on the deposited feature.

Chapter 5 presents and discusses the morphology, microstructure and crystal formation mechanism of the films as affected by printing condition.

Chapter 6 presents and discusses the results of electrical characterization with and without UV excitation.

Chapter 7 concludes the thesis and suggests future directions of research to be carried out. 


\section{LITERATURE BACKGROUND}

\subsection{Direct Writing (DW)}

DW, also referred to as direct printing or digital printing, as a form of micro/nano scale additive manufacturing technique is a diverse, versatile and scalable group of process technologies [12]. A couple of definitions have been put forward to describe what DW entails by various scholars. Pique and Chrisey [17] defined DW as any technique or process capable of depositing, dispensing or processing different types of materials over various surfaces following a preset pattern or layout. Also, Hon et al [13] defined it to be a group of processes, which are used to achieve deposition of functional and /or structural materials on a substrate in digitally defined locations. Additionally, it was defined as the fabrication method that employs a computercontrolled translation stage, which moves a pattern generating device, e.g. ink deposition nozzle or laser writing optics, to create materials with controlled architecture and composition by Lewis and Gratson [18]. Another and more expansive definition was given by the British Department of Trade and Industry (DTI), where DW was described as a range of technologies which allow the fabrication, possibly in reconfigurable short production runs, of two- or three-dimensional functional structures using processes that are compatible with being carried out directly onto potentially large complex shapes [19].

While most these definitions have partially different views of what DW encompasses, they all share some common grounds. The first is that DW can be used to selectively deposit materials in any desired location. Also, various types of materials can be deposited and that a substrate, which forms an integral part of the device, is required. Another common point is that complex shapes in two or three dimensions can be directly written and most importantly, the geometry of the deposited features can be controlled. 
Moving away from what DW is believed or supposed to be, and concentrating more on the benefits derivable from the use of these technologies, it can be argued that DW has contributed immensely to various industries. DTI [19] supports the thesis that these technologies have the potential of revolutionizing manufacturing in many sectors including automotive, pharmaceuticals, information and communication technology, ceramics and biotechnologies and to lead, among many other benefits, to the reduction of production costs and manufacturing times. To be more specific, DW enables high resolution, on the order of 10 to $1000 \mathrm{~nm}$, selective deposition and patterning of materials in single- and multiple layers on various surfaces [20]. Church et al. [21] stated that DW enables rapid manufacturing of sensors and antennae because it eliminates the masking and etching steps of conventional electronics fabrication. Lewis, [22] reported that direct write assembly allows design and rapid fabrication of materials in complex 3D shapes without the need for expensive tooling, dies, or lithographic masks. Use of environmental friendly, disposable and cheap materials is another advantage that makes these technologies more attractive in this day of great concern on environmental pollution [12]. A comparison of DW technologies with the conventional electronic fabrication techniques by Engstrom et al. [23] shows that, they possess moderate to high patterning speeds $\left(10^{-10}\right.$ to $\left.10 \mathrm{~m}^{2} / \mathrm{hour}\right)$, and resolution (10 to $1000 \mathrm{~nm}$ ). The capital cost of equipment required for direct writing was among the lowest possible as depicted in the comparison in Figure 2.1.

\subsection{Classification of DW}

Despite the fact that all DW technologies serve the same function of selective deposition of conductive traces, the means of realizing this goal varies from one technology to another. Therefore, their classification, just like the definition, had been done differently and is often based 
on a couple of parameters, which include: method of pattern transfer, scale of operation, and deposition environment [13] [24] [25]. Adopting the classification based on the mechanism of materials transfer presented by Hon et al. [13], DW methods can be broadly divided into four groups as: droplet based, energy beam based, flow based and tip based as shown in figure 2.2.

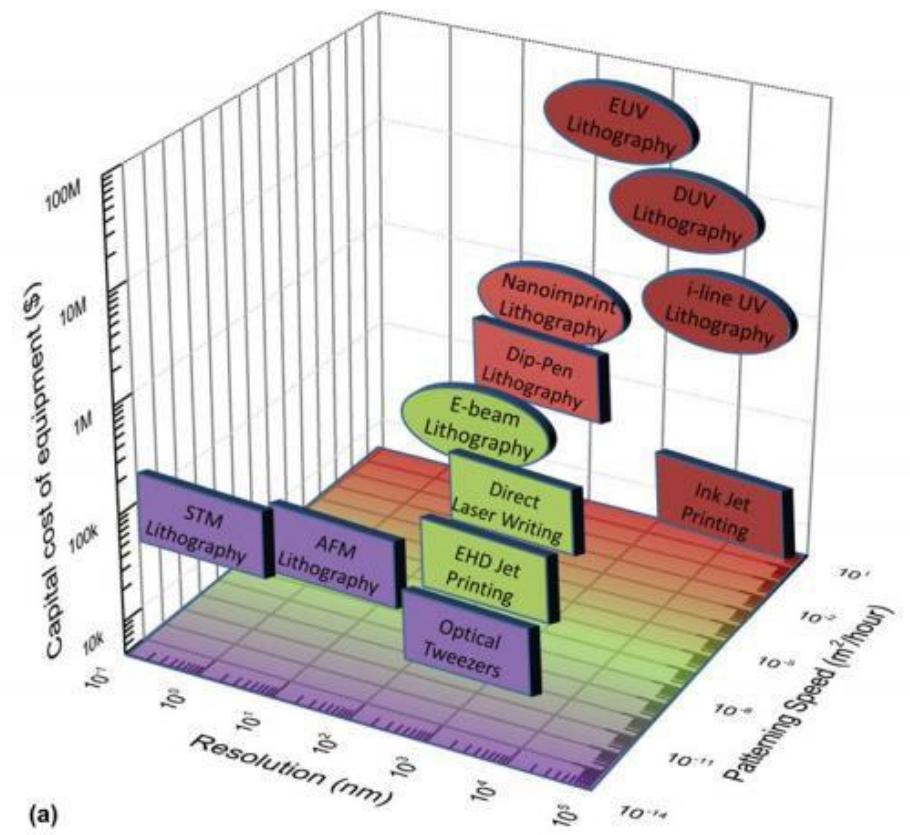

Figure 2.1 Pictorial representation of DW comparison with other electronic fabrication methods [23]

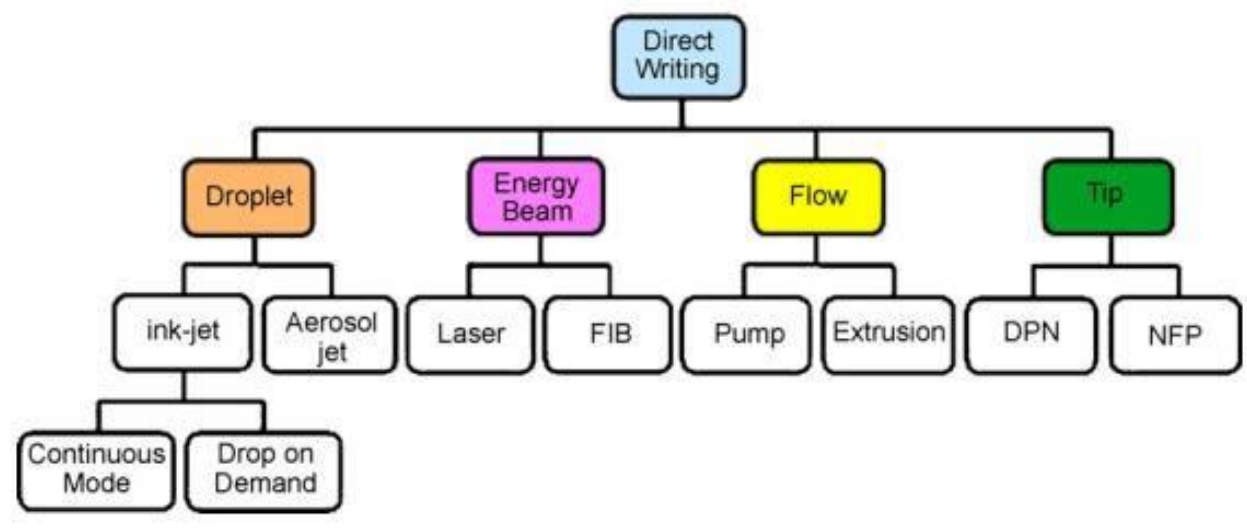

Figure 2.2 Schematic representation of the classification of direct writing [13] 
Typical examples of the droplet based DW method are the ink jet and aerosol jet printing methods. The ink jet printing which can be operated in continuous or in drop-on-demand mode, involves the formation and deposition of a sequence of droplets of liquid material, often called an ink or fluid. It uses either low viscosity fluids that must be removed by absorption and evaporation or wax-based inks that are heated during droplet formation and then solidify upon impact cooling [26]. In aerosol jets, unlike inkjet systems, the driving force is based on a gas providing the kinetic energy for the deposition of materials. Aerosol jetting systems aerosolize a material to create a gaseous stream that is aerodynamically focused and deposited on the substrate [27]. Energy beambased DW involves the use of laser or ion beams to deposit materials. The laser-based systems use the action of a high-energy laser beam to transfer strip of material onto a substrate surface. This method can be further subdivided into four groups based on the precursors, i.e. solid, liquid, gas or cell [28]. Focused ion beam DW requires a precursor gas and has higher resolution, but slower writing speed when compared with laser based DW [29]. Tip-based deposition methods use capillary flow of an ink on microscale tip onto the substrate surface and it includes: DPN where molecules diffuse on to a substrate in an ordered pattern through the micro-capillary action between the tip and the surface [10], and the nano fountain, in which a cantilever nanopipette is used in place AFM tip.

In flow-based DW, high precision micro-dispensing technology, which could be in the form of a precision pump or extrusion, is used to force materials through a very small orifice or a needle. Its major advantage lies in its ability to handle materials over a wide range of viscosities from 0.5 to $1,000,000 \mathrm{mPas}$ [13]. Extrusion type flow-based DW technologies, which is the focus in this work, uses positive pressure for dispensing materials in fluid form through a small nozzle. Since pressure, dispensing height, speed, material type, affects the properties of features being 
printed through this process, precise control over these parameters is important [15. Its capability to rapidly fabricate materials in complex three-dimensional shapes without the need for expensive tooling, dies, or lithographic masks, makes it a promising (nano/micro) manufacturing method.

\subsection{Applications of DW Techniques in Device Manufacturing}

There is an enormous variety of products in electronics, medical devices, energy harvesting and storage, environment and health sciences, where the simplicity and versatility of DW techniques has been annexed. Tissue engineering, microfluidics, orthopedics, photovoltaics, optical waveguides and sensing devices, to mention a few, are some of the specific high tech devices where direct writing has been applied [23].

Eqtesadi et al. [30] fabricated a complex 3D scaffold for bone tissue engineering application through robotic deposition (robocasting) using 45S5 bioactive glass. They reported strength values that are substantially higher than any previously reported data for 45S5 Bioglass scaffolds. They therefore, confirmed the suitability of robocasting technique for producing vitreous 45S5 scaffolds with a sufficient mechanical integrity for any practical application. They also reported on the capability of the process for developing 45S5 Bioglass scaffolds with customized external geometry, and optimized pore architecture. Dingledein et al. [31] performed a process characterization of optical multi-mode waveguides fabricated with polymer using the direct dispensing method. They reported total optical losses in the range of $0.0-0.09 \mathrm{~dB} / \mathrm{cm}$ at $850 \mathrm{~nm}$ for the fabricated waveguides compared to $<0.05 \mathrm{~dB} / \mathrm{cm}$ for lithographically fabricated waveguides. It was further stated that the ability to directly dispense waveguides reduced production cost by eliminating material waste and the need for costly photo-masks. 
Noh et al. [32] and Bucella et al. [33] both used inkjet writing in producing an all printed polymer thin film transistor (TFT) and organic field effect transistor (OFET) metallic electrodes, respectively. Noh et al. [32] reported the printing of a device with architecture that minimizes contact resistance effects and gate configuration that minimizes parasitic overlap capacitance to values as low as $0.2-0.6 \mathrm{pFmm}^{-1}$, and allows reaching a transition frequency of $51.6 \mathrm{MHz}$. Bucella et al. [33] combined laser ablation with inkjet writing and reported producing arrays of tens of devices with good uniformity, and concluded that robustness of the DW approach led to the patterning of downscaled electrodes for high performance OFETs.

A bulk-heterojunction (BHJ) solar cell was efficiently printed by Lee et al. [34] using inkjet printing technology with conventional chlorobenzene solution as ink. They reported obtaining the best BHJ solar cell structure at around $50 \%$ of droplet overlaps with $2 \mathrm{wt} \% \mathrm{BHJ}$ ink at $25^{\circ} \mathrm{C}$ of substrate temperature. Maximum power conversion efficiency of $2.83 \%$, with an open circuit voltage of $0.62 \mathrm{~V}$, a short circuit current density of $8.60 \mathrm{~mA} / \mathrm{cm}^{2}$, and a fill factor of 0.53 under air mass $1.5 \mathrm{G}$ irradiation $\left(100 \mathrm{~mW} / \mathrm{cm}^{2}\right)$ were also reported. Sun et al. [35] produce a Li-Ion microbattery through three dimensional printing. They printed multiple layers of high aspect ratio filaments from concentrated inks, containing $\mathrm{Li}_{4} \mathrm{Ti}_{5} \mathrm{O}_{12}$ and $\mathrm{LiFePO}_{4}$ on gold coated glass substrate, to serve as anode and cathode, respectively. They reported obtaining desirable porous structure after annealing, which allows electrolyte penetration. Electrical resistivities of $2.1 \times 10^{5}$ $\Omega \mathrm{m}$ and $2.3 \times 10^{3} \Omega \mathrm{m}$ were reported for the printed anode and cathode, respectively. A three dimensional quantum dot light-emitting diodes was printed on contact lens by Kong et al. [36] by seamlessly interweaving five different materials. They demonstrated ability for printing on curvilinear surface, a feat not achievable by using standard microfabrication techniques. The printed QD-LEDs was reported to exhibit pure and tunable color emission properties. 


\subsection{Extrusion Type, Flow-Based DW Parameters}

The efficient printing of material features through any DW technique and more specifically by nozzle based direct writing (NBDW) is influenced by some factors. These factors can have direct or indirect effects on both the physical and structural properties of the printed feature [15]. The factors or parameters can be categorized into two groups, which are material parameters and process parameters. The material parameters are determined by the properties of the material (usually called 'ink') to be printed. Inks are often formulated by mixing solvent, precursor and additives in proportions that yields printable colloids or gels, and its important properties include viscosity, surface energy and elasticity [30]. The process parameters, in the case of NBDW technique include the air pressure, the inner diameter of the printing nozzle, the dispensing height i.e. the nozzle-substrate clearance, and the speed at which the nozzle traverse the substrate surface.

In order to achieve efficient feature printing, optimal combination of these parameters is a necessary requirement. Inks with appropriate rheological properties are matched with process parameters, such that, ink flows out under pressure and take a desired shape upon exit from the nozzle. Various authors have attempted controlling the formulation of the printable ink and matching it to appropriate process parameters in order to study their combined effects on the printed features. Bruneaux et al. [37] reported on the characterization of extruded organic fugitive ink made from mixture of petroleum jelly and wax. They estimated process apparent viscosity and the complex viscosity from the experiment, and found that the former is up to four orders of magnitude smaller than the later. They concluded that, in order to optimize the resulting shape of the microchannel, the translational speed of the moving stage has to closely match the linear velocity of the fugitive ink at the micro-nozzle exit. Ahn et al. [15] [16] demonstrated the printing 
of ITO and silver microelectrodes, respectively, through direct write assembly and showed that with selection of appropriate material and process parameters, the microelectrode can be printed both on a planar substrate surface and across gaps.

\subsection{Structure, Properties and Applications of Zinc Oxide (ZnO) Thin Film}

$\mathrm{ZnO}$, is an extremely promising material for the development of optoelectronic devices and it is a group II-VI binary compound semiconductor. It exhibits typical sp3 covalent bonding, but, shows iconicity at the borderline of covalent and ionic semiconductors [38]. It has high thermal and chemical stability, high mechanical strength, and possesses a highly crystalline hexagonal structure [39]. It is capable of being grown at low temperature, being nontoxic to the environment and transparent in the visible range [40].

It crystallizes majorly in either cubic zinc blende or hexagonal wurtzite with the later representing the thermodynamically stable phase under ambient conditions [38]. The nonpolar (1010) surface represents $\mathrm{ZnO}$ 's most stable surface, however, $\mathrm{ZnO}$ film with wurtzite structure has been found to have preferential growth direction along the c-axis with exposed polar surfaces identified as (0002) or (0001) [41]. Also, it can easily be manipulated to exhibit different nanostructures such as nanowires, nanopores, nanorods, nanobelts, nanorings, nanotubes, and so on [39]. 


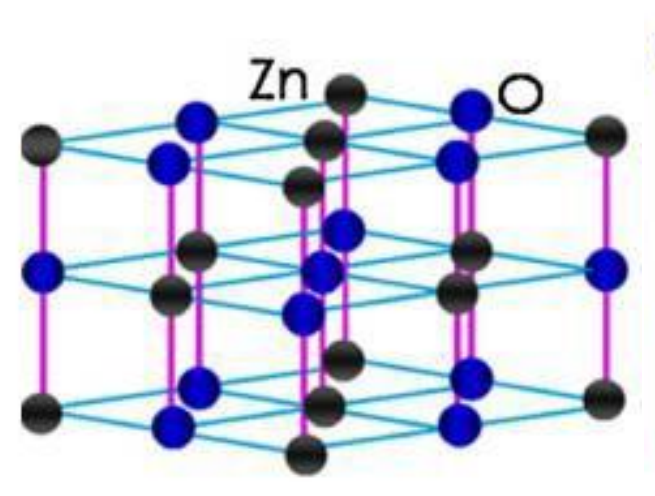

(a)

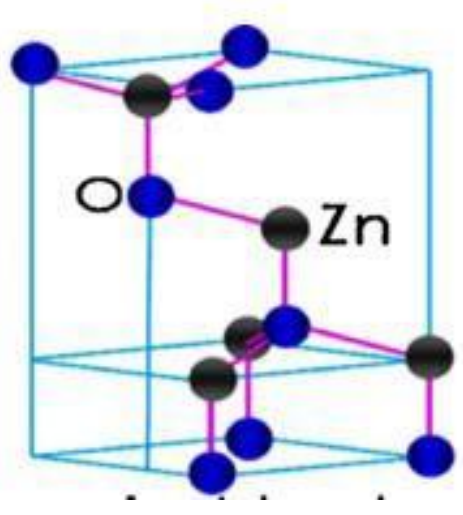

(b)

\section{Figure 2.3 (a) wurtizite and (b) Zincblende, crystal structure of $\mathrm{ZnO}$}

The band structure of any semiconductor is pivotal in determining its potential application. $\mathrm{ZnO}$ has wide and direct band gap with band gap energy of approximately $3.37 \mathrm{eV}$ at room temperature [42]. The band gap energy of $\mathrm{ZnO}$ is tunable to between 7.9 and $2.3 \mathrm{eV}$ spanning from deep ultraviolet (UV) to visible regions of the spectrum, by alloying with $\mathrm{MgO}$ and $\mathrm{CdO}$ [43]. It has a large exciton binding energy of approximately $60 \mathrm{meV}$, higher than other wide band gap semiconductors like GaN and SiC. The large exciton binding energy enables efficient and stable excitonic transitions at room temperature. $\mathrm{ZnO}$ also shows quantum confinement effect i.e. variation in band gap of semiconductor with particle size [44].

Myriad areas of applications have been reported in literature for $\mathrm{ZnO}$ due its fascinating properties with its applications cutting across different areas in microelectronics, optoelectronics, photonics and spintronics. $\mathrm{ZnO}$ is an extremely promising material for the development of optoelectronic devices, such as light emitting and laser diodes, solar-blind photo detectors, and transparent thin film transistors. Applications such as chemical and gas sensors, due to the large 
surface to volume ratio, and microlasers due to its superior optical properties have been reported [45]. Furthermore, reports of high-temperature ferromagnetism in $\mathrm{ZnO}$ doped with transition metals (known as dilute magnetic oxide semiconductor) [46] coupled with nanosecond-long spin coherence time measured at low temperatures makes $\mathrm{ZnO}$ an extremely promising material for future application in spin electronics.

\subsection{Sol-Gel Synthesis of Pure and Doped ZnO}

Sol-gel method is fast becoming a method of choice for synthesizing various functional metal oxide films. The advantages of this route include the use of simple deposition methods and ease of doping with other materials. $\mathrm{ZnO}$ thin film by sol-gel process is usually achievable through two chemical routes: the alkoxide and the non-alkoxide routes. In the alkoxide precursors based process, dimethyl zinc or diethyl zinc are often used. These reagents are highly reactive and expensive, and the precursor solution is extremely sensitive to moisture. For the non-alkoxide route, $\mathrm{Zn}$ salts, such as acetates, are dissolved in water or alcohol and ethanolamine is used as a sol stabilizer [47].

Cronin et al. [10] synthesized $\mathrm{ZnO}$ inks through a sol-gel process using Zinc acetate dehydrate, isopropyl alcohol and monoethanolamine as precursor, solvent and stabilizer, respectively, and stirred at $60{ }^{\circ} \mathrm{C}$ for $2 \mathrm{~h}$. They also added glycerol to act as a thickening agent. $\mathrm{ZnO}$ thin films were prepared through sol-gel method by Khodja et al. [48] dissolving zinc acetate dihydrate in a mixture of absolute ethanol and monoethanolamine. They varied the molar ratio of monoethanolamine to zinc acetate from 0.5 to 2.0 while vigorously stirring at $50^{\circ} \mathrm{C}$ for one hour. Chopali et al. [49] used the modified Pechini (alkoxide) process to prepare $\mathrm{ZnO}$. In this process, 
$0.1 \mathrm{~mol} \mathrm{Zn}(\mathrm{NO} 3)_{2}, 0.9 \mathrm{~mol}$ glycerol, and $0.1 \mathrm{~mol}$ of $\mathrm{HNO}$, were added together. Deionized water was added to the solution and the resulting solution was heated at a constant temperature of $70^{\circ} \mathrm{C}$, while stirring continuously to synthesize a clear, homogeneous solution. Foo et al. [39] prepared $\mathrm{ZnO}$ seed solution by using zinc acetate dehydrate as a precursor, methanol, ethanol, isopropyl alcohol and 2-methoxy ethanol as solvent and monoethanolamine as a stabilizer. They stirred the solution at $60^{\circ} \mathrm{C}$ for 2 hour and subsequently stored it at room temperature for 24 hours to age.

AZO was prepared by Gahtar et al. [50], O'Brien et al. [51] and Gao et al. [52]. They all used zinc acetate dehydrate as precursor, and monoethanolamine was used as stabilizing agents by O’Brien et al. [51] and Gao et al. [52], while, Gahtar et al. [50] used HCL to stabilize the solution. The aluminum doping was obtained from aluminum chloride, by Gahtar et al. [50], aluminum nitrate by O'Brien et al. [51] and aluminum chloride hexahydrate by Gao et al. [52]. The solvents used were an equal volume of ethanol and methanol solution [50], isopropanol [51], and ethanol [52]. The resulting solutions were stirred at temperatures ranging from 50 to $70^{\circ} \mathrm{C}$ for one to three hours and aging time from 24 to 48 hours.

Manganese doped $\mathrm{ZnO}$ was synthesized by Xin et al. [40] and Mimouni et al. 2015. Xin et al., 2014 used Zinc acetate dehydrate, Ethylene glycol monomethyl ether and monoethanolamine as the precursor, solvent and stabilizing agent, respectively. Mn doping was achieved by adding manganese chloride $\left(\mathrm{MnCl}_{2}\right)$ in varying quantities after which the solution was stirred for two hours and aged for 24 hours. Mimouni et al. [53] synthesized ZnO, contained zinc acetate dihydrate, mixture of water and propanol in varying ratios, acetic acid and Magnesium chloride tetrahydrate keeping the Mn-to-Zn molar ratios between 0 to $3 \%$.

Rozati et al. [54] prepared fluorine doped $\mathrm{ZnO}$ by dissolving zinc acetate dehydrated in a mixture of double distilled water, methanol (3:7 volume proportion) and acetic acid. Doping of the 
films was achieved by adding ammonium fluoride to the starting solution, with a fixed $[\mathrm{F}] /[\mathrm{Zn}]$ ratio of 2 at.\%. Shewale et al. [55] synthesized Ti-doped $\mathrm{ZnO}$ thin films through chemical solution by mixing appropriate volumes of equimolar $(0.05 \mathrm{M})$ solution of high purity zinc acetate and titanyl acetylacetonate in methanol.

\subsection{ZnO Thin Film Deposition and Characterization Methods}

Various physical and chemical techniques have been employed for depositing $\mathrm{ZnO}$ thin films on different kind of substrates. Many of the major deposition techniques for $\mathrm{ZnO}$ include thermal evaporation and condensation [56], chemical vapor deposition [57], metal organic chemical vapor deposition [58], spray pyrolysis [3], electrodeposition [59], molecular beam epitaxy [60], pulsed laser deposition (PLD) [61], chemical bath deposition [62], magnetron sputtering [7], and sol-gel deposition [47]. ZnO prepared through sol-gel process is used in conjunction with other film deposition techniques like spin coating [8], dip coating [63] and direct writing [10] [11]. The sol-gel method has attracted much attention due to its simplicity, safety, low cost, controllability of compositions, large-area deposition, and uniformity of thickness [40] [47]. It also offers an easy and efficient way of doping with other materials, for the purpose of improving the base materials' inherent properties or introducing new ones.

Deposited films through any method requires extensive and detailed characterization in term of their structural, morphological, optical, and electrical properties to define their state and potential applications. Various characterization techniques are used and include optical and SEM, and XRD spectroscopy for determining microstructure, ultra violet-visible-near infra-red (UV- 
VIS-NIR) spectroscopy and photoluminescence (PL) for optical properties and semi-conductor analyzer for electrical properties.

The properties of $\mathrm{ZnO}$ thin films deposited by spin coating materials with different solvents was determined by Foo et al. [64]. The morphology, crystallization and microstructures of the $\mathrm{ZnO}$ thin films were determined by using field emission scanning electron microscopy (FESEM), atomic force microscopy (AFM) and XRD. A Fourier transform infrared (FTIR) was used to analyze the elemental stretching vibration and optical transmittance measurements were carried out using UV-VIS-NIR spectrophotometer. They reported that the thin film prepared using 2methoxy ethanol has the smallest grain size, highest transmittance ( $>90 \%)$, lowest surface roughness of $3.131 \mathrm{~nm}$, and highest band gap energy of $3.28 \mathrm{eV}$. Also, it was reported that the obtained $\mathrm{ZnO}$ films were polycrystalline with preferred orientation along the (002) plane, while, isopropyl alcohol derived films have a preferred orientation on (101) plane.

O'Brien et al. [51] studied the effect of dopants on the morphology, microstructure and electrical properties of spin coated and inkjet printed transparent $\mathrm{ZnO}$ films. The films obtained were characterized using XRD, SEM, AFM, UV-VIS spectrophotometer and Keithley 617 electrometer. They reported crystal growth of the non-doped $\mathrm{ZnO}$ to be in the 100, 002 and 101 directions while the Al-doped $\mathrm{ZnO}$ has preferential growth in the 002 plane, with the conductivity reaching a maximum value for $\mathrm{ZnO}$ containing $2 \% \mathrm{Al}$, and sintered at $475^{\circ} \mathrm{C}$. Xin et al. [40] and Mimouni et al. [53] produced thin film of $\mathrm{Mn}$ doped $\mathrm{ZnO}$ by spin coating/water bath treatment and by chemical spray technique, respectively. They both studied the effects of manganese element content on structural, optical, photoluminescence, opto-thermal and electrical conductivity of $\mathrm{ZnO}: \mathrm{Mn}$ thin films by using of XRD, photoluminescence spectroscopy and impedance spectroscopy. All the films reported consist of single phase with the crystallites preferentially 
oriented towards (002) plane parallel to c-axis. Manganese doping resulted in slight decrease in the optical band gap energy of the films, with noticeable change in optical constants, and a slightly red shift to the longer wavelength in the position of the UV peak.

Axelevitch et al. [65] investigated the properties of $\mathrm{ZnO}$ thin films prepared by sputtering methods in both pure argon and argon-nitrogen mixture atmospheres. An optical microscope, scanning electron microscope, standard four-point probe electrical measurement and photo spectrometer were used for characterizing the film. They reported transparent, low-resistive, ntype conductivity films with polycrystalline structure in the c-axis direction. Conductivity and transparency at $550 \mathrm{~nm}$ wavelength were found to be approximately $1.8 \times 10^{-2} \Omega \mathrm{cm}$, and $82.1 \%$ respectively. Radio frequency magnetron sputtering was used in depositing $100 \mathrm{~nm}$ thick films of $\mathrm{ZnO}$ onto a glass substrate from different targets by Prepelita et al. [7]. Polycrystalline films possessing wurtzite (hexagonal) structure, with crystallites preferentially oriented in (002) plane, and with surface roughness, between $1.7 \mathrm{~nm}$ and $2.7 \mathrm{~nm}$ were reported. The mechanism of electrical conduction in the studied films was reported to be strongly influenced by the films' polycrystalline structure. Thermal activation energy of electrical conduction of the samples vary from $1.22 \mathrm{eV}$ to $0.63 \mathrm{eV}$ for different targets, and optical band gap determined from absorption spectra varied between $3.28 \mathrm{eV}$ and $3.25 \mathrm{eV}$.

Gahtar et al. [50] produced and characterized thin films of pure and $\mathrm{Al}$-doped $\mathrm{ZnO}$ by ultrasonic spray method using ethanol and methanol as solvents. They determined the crystallographic and phase structures of the thin films by XRD, the optical properties in the range of 300-800 nm by a UV-VIS spectrophotometer, and the electrical conductivity of the films by a four probe method. They reported obtaining $\mathrm{ZnO}$ films that have nanocrystalline nature and hexagonal wurtzite structure with (100) and (002) preferential orientation. An average optical 
transparency of about $90 \%$ and band gaps values of $\mathrm{ZnO}$ thin films, which increased after doping from 3.10 to $3.26 \mathrm{eV}$ and 3.27 to $3.30 \mathrm{eV}$, with conductivity ranging from 7.5 to $15.2 \Omega / \mathrm{cm}$, were also reported. Shewale et al. [55] produced thin films of un-doped and Ti-doped $\mathrm{ZnO}$ by chemical spray pyrolysis technique. They studied the influence of Ti doping on structural, morphological, optical and UV detection properties of the obtained ZnO film by using XRD, FESEM, UV-VISNIR and Keithley instruments model 2410 Sourcemeter. The films showed polycrystalline nature with a hexagonal wurtzite structure; and improved film crystallinity was obtained by Ti doping. Surface morphological observations agreed well with structural results. The Ti doping increased the optical transmittance $(96 \%$ at $550 \mathrm{~nm}$ ) and band gap (3.2927 eV) of $\mathrm{ZnO}$ thin film.

\subsection{Post Processing of Deposited ZnO Thin Films}

$\mathrm{ZnO}$ thin films, after deposition are often required to be dried and subsequently sintered to become functional. Sintering, which is usually the final processing step, is achieved by heating the deposited film, in air or under controlled atmosphere. The maximum heating temperature applied, the heating rate, how long the sintering temperature is maintained, the cooling rate and the environment in which the sintering was carried out, contributes to determining the intrinsic and extrinsic properties of the functional film produced. Negative affect due to stress induction, material oxidation or substrate warping can result due to improper combination of the sintering parameters. Densification and grain growth, which occurs during the sintering process, is controlled by the mode of sintering applied, and thus, contributes to the crystal structure and ultimately determines the films applicability. Different methods of sintering are applied in thin film sintering; the traditional method involves heating to a high temperature in a furnace until 
crystallization is achieved. Other methods include electric field assisted sintering, and laser sintering.

Babu et al. [44] sintered a mixture of $\mathrm{Zn}(\mathrm{NO} 3) 26 \mathrm{H} 2 \mathrm{O}$, water and PVA at temperature of $600,700,800$ and $900^{\circ} \mathrm{C}$ to obtain $\mathrm{ZnO}$ nanoparticle powder. The resulting $\mathrm{ZnO}$ powder characterized by carrying out a structural and photoluminescence analysis gave hexagonal crystal structure showing reflections in (100), (002), (101), (102), (110) and (103) planes and has mean grain size values ranging from 51 to $71 \mathrm{~nm}$. Effect of heating rate on the structural and electrical properties of sol-gel derived Al-doped $\mathrm{ZnO}$ films, deposited on glass substrate by spin coating was studied by Gao et al. [52]. They sintered AZO films by heating $550{ }^{\circ} \mathrm{C}$ in air using heating rates of $10,20,40$ and $50{ }^{\circ} \mathrm{C} / \mathrm{min}$ after pre heating at $300{ }^{\circ} \mathrm{C}$ for 10 minutes. Heating at a high rate of $50 \circ \mathrm{C} / \mathrm{min}$ resulted in formation of nanorods.

\subsection{ZnO Thin Film Electrical Conductivity and UV Detection}

The electrical performance of any transparent conducting oxide is most directly related to its carrier concentration and its carrier mobility [66]. The optimal value for both can vary depending on the intended application for which the TCO is prepared. $\mathrm{ZnO}$ being a natural n-type semiconductor conducts electricity by the transport of electrons, which are the major carriers in ntype semiconductors. The electrical properties of $\mathrm{ZnO}$ are also said to be dependent on the composition and on microstructural characteristics, such as grain size, density, morphology and the distribution of second phases. Currently, research effort is directed at understanding and overcoming factors responsible for the low electrical performance exhibited by $\mathrm{ZnO}$-based materials prepared through commercially relevant techniques. 
In a bit to improve the electrical performance of $\mathrm{ZnO}$, excitation and activation of the electrons of both bulk and thin film $\mathrm{ZnO}$ with high energy beam has been applied, and has become a well-established phenomenon. This property, which enables the electrical conductivity of $\mathrm{ZnO}$ to change upon irradiation, has been exploited for sensing and catalysis applications. The effects of the irradiation are often found to decay over a timescale, when the irradiated sample is exposed to air.

Vai et al. [66] reported the occurrence of long-lasting enhancement in electrical properties when $\mathrm{ZnO}$ thin films prepared by spray pyrolysis in a nitrogen atmosphere were treated with ultra violet (UV) light prior to the films' first exposure to air. They obtained a record carrier mobility of $44.3 \mathrm{~cm}^{2} \mathrm{~V}^{-1} \mathrm{~s}^{-1}$ and attributed the improved electrical properties to the modification of the relative populations of donor and acceptor defects at the grain boundaries of the irradiated polycrystalline films. Wu et al. [67], produced a metal-semiconductor-metal photodetectors by depositing $\mathrm{ZnO}$ and $\mathrm{MgZnO} / \mathrm{ZnO}$ using radio frequency sputtering method, treated the film with atmospheric pressure plasma jets and irradiated with $368 \mathrm{~nm}$ wavelength UV light. They reported an increase in dark current and photocurrent levels, photoresponsivities, and photocurrent response times in the fast rising and decay transition with the APPJ treatment duration. They attributed the observations to the shielding of ambient oxygen, defect and surface states passivation, and introduction of highly conductive interfaces at the $\mathrm{MgZnO} / \mathrm{ZnO}$ heterojunctions.

An un-doped and $\mathrm{Al}$-doped $\mathrm{ZnO}$ thin films deposited onto glass substrates by the sol-gel spin coating method was characterized for its photoconductivity after irradiation with $366 \mathrm{~nm}$ wavelength UV light by Mahroug et al. [68]. They reported an increase in measured current after excitation with UV light and attributed the response in photocurrent to photo excitation of electrons from valence band into conduction band. They found that the minimum photocurrent was produced 
by un-doped $\mathrm{ZnO}$ thin film, while, maximum photocurrent was obtained for 3\% $\mathrm{Al}$ doped $\mathrm{ZnO}$ thin film. Shewale et al. [55] investigated the room temperature UV photo detection properties of pulse laser deposited Magnesium doped $\mathrm{ZnO}$ films in metal-semiconductor-metal planar configurations. The photocurrent was reported to have increased with reduction in oxygen pressure and increase in exposure time. The improved electrical property was considered to be due to the enhancement in film crystallinity and oxygen vacancies. They also, reported on achieving a highly stable and fast switching UV photoresponse characteristics for the film prepared at $2 \times 10^{-3}$ torr oxygen pressure upon exposure to a $2 \mathrm{~mW} / \mathrm{cm}^{2} \mathrm{UV}$ at $365 \mathrm{~nm}$.

Bera and Basak [69], synthesized $\mathrm{ZnO}$ nanowires with a $\mathrm{ZnS}$ coating. Both nanowires exhibited similar dark current values, whereas the photocurrent value was reported to be much higher in the surface-modified NWs. The higher photocurrent value was said to indicative of transport of the photogenerated carriers from the $\mathrm{ZnS}$ layer to $\mathrm{ZnO}$ during $\mathrm{UV}$ illumination. The photocarrier relaxation under steady UV illumination was also reported to occur in $\mathrm{ZnO}$ nanowire arrays but absent in $\mathrm{ZnO}$ coated with $\mathrm{ZnS} \mathrm{NW}$ arrays. Bao et al. [70] also worked on photoconductivity of individual $\mathrm{ZnO}$ nanowires and found that the induced photocurrents persist both in air and in vacuum. They observed that the photoresponse in vacuum was much greater than that obtainable in air. Photoinduced surface depletion depth decrease caused by oxygen desorption induced by photogenerated holes was said to be responsible for the observed behavior. Hullavarad et al. [71] investigated the origin of persistent photoconductivity by using a 30-65 $\mathrm{nm}$ diameter and $5 \mu \mathrm{m}$ long $\mathrm{ZnO}$ nanowire in metal-semiconductor-metal photoconductor device. They took the current-voltage measurements with and without UV illumination under different oxygen levels and found that persistent conductivity trend exists for depleted oxygen conditions. 


\section{EXPERIMENTAL METHODS AND MATERIALS}

\subsection{Substrate Preparation and Cleaning}

Soda lime microscope glass slide is used as substrate for printing. It was cut into required sizes by using a diamond tip glasscutter. Subsequently, the glass slides were cleaned according to the following procedure: the glass slides were placed in beaker filled with ethanol (Decon Laboratories Ethanol 200 proof) and was ultrasonically agitated for one hour. This was followed by sonication for one hour in acetone (ACS Grade S2512OA) and another additional hour of sonication in deionized (DI) water. The cleaned glass slides were then dried in an electric oven at $150{ }^{\circ} \mathrm{C}$ for one hour, after which they were kept in a sealed container to prevent accumulation of dirt on the surface. A Brandson 2510 ultrasonic bath was used for all ultrasonic cleaning and mixing in this work.

\subsection{Synthesis of ZnO Sol-Gel}

$\mathrm{ZnO}$ sol gel was synthesized through various methods based on the precursors, solvents, and stabilizer used. However the synthesis of both un-doped and doped $\mathrm{ZnO}$ were carried out through the solution method.

\subsubsection{Un-doped $\mathrm{ZnO}$}

In the earliest part of this work, $\mathrm{ZnO}$ sol gel containing DI water, polyvinyl pyrolidone $(\mathrm{PVP})(\mathrm{M} . \mathrm{W} .=1,300,000)$ and zinc acetate dihydrate $\left(\mathrm{Zn}\left(\mathrm{CH}_{3} \mathrm{OO}\right)_{2} \cdot 2 \mathrm{H}_{2} \mathrm{O}\right)$ was prepared as follows: 
Desired quantity of PVP was slowly added to appropriate quantity of DI water while stirring on magnetic stirrer (Fisher Scientific Isotemp-Stirer) at room temperature. After the PVP addition, vigorous stirring of the mixture continued for 24 hours at room temperature to ensure complete dissolution. ZAD (2.25 g), which constitutes $15 \mathrm{wt} \%$ of the entire solution, was added to the solution and stirring continued for an additional 8 hours. The solution was then sonicated for 2 hours to further enable mixing and dissolution of the ink. Three variants of the solution named inks $\mathrm{A}, \mathrm{B}$ and $\mathrm{C}$ were prepared following dissolution of DI water-PVP-ZAD in ratios of 14:3:3, 13:4:3 and 12:5:3 by weight respectively. The composition of each ink is shown in Table 3.1.

Table 3.1: Composition of different un-doped $\mathrm{ZnO}$ inks used in this study

\begin{tabular}{cccc}
\hline \hline Ink & DI water, g(wt\%) & PVP, g(wt\%) & Zinc Acetate Dihydrate, g(wt\%) \\
\hline A & $10.5(70)$ & $2.25(15)$ & $2.25(15)$ \\
B & $9.75(65)$ & $3.0(20)$ & $2.25(15)$ \\
C & $9.0(60)$ & $3.75(25)$ & $2.25(15)$ \\
\hline
\end{tabular}

\begin{tabular}{|c|c|c|c|c|}
\hline $\begin{array}{l}\text { PVP gradually } \\
\text { added to DI } \\
\text { water }\end{array}$ & $\begin{array}{l}\text { Stirring } \\
\text { for } 24 \\
\text { hours }\end{array}$ & $\begin{array}{l}\text { Adding } \\
\text { ZAD }\end{array}$ & $\begin{array}{l}\text { Stirring } \\
\text { for } 8 \\
\text { hours }\end{array}$ & $\Rightarrow \begin{array}{l}\text { Sonicating } \\
\text { for } 2 \text { hours }\end{array}$ \\
\hline
\end{tabular}

Figure 3.1: Schematic of the un-doped $\mathrm{ZnO}$ preparation process. 


\subsubsection{Synthesis of AZO Sol-Gel}

Due to challenges encountered with the un-doped, water based $\mathrm{ZnO}$ synthesized through the process described above; an alternative synthesis route was adopted. The challenges encountered include: the deposited feature not forming continuous film after sintering, weak adhesion of sintered film to glass substrate, and very high resistance to current flow. In overcoming these problems, AZO was synthesized by using ZAD and Aluminum Nitrate as precursors, while, 2-Methoxy Ethanol (ACROS ORGANIC, 99\% extra pure) and Ethanolamine (Alfa Aesar ACS 99\%) were used as solvent and stabilizer respectively.

A mixture of Aluminum Nitrate and ZAD used as precursor for the solution consists of 3 wt $\%$ of Aluminum Nitrate and $97 \mathrm{wt} \%$ ZAD. This was achieved by adding $0.1 \mathrm{~g}$ of Aluminum Nitrate to $3.5 \mathrm{~g}$ of ZAD in a container and manually mixed together. The solution was formed by dissolving the measured precursor in $16 \mathrm{~mL}$ of 2-Methoxy Ethanol and $2 \mathrm{~g}$ of Ethanolamine added thereafter. The entire solution mixture was vigorously stirred for 2 hours on a magnetic stirrer (Fisher Scientific Isotemp-Stirer) while heating at $60{ }^{\circ} \mathrm{C}$ to aid dissolution. The mixed solution was subsequently allowed to age by keeping at room temperature for 24 hours.

In order to enable printability of the solution, PVP was added to control the viscosity. Inks with different viscosities were obtained by adding PVP in varying quantities of 10, 15 and $20 \mathrm{wt} \%$ of the solution. The mixtures containing the PVP were further stirred for 24 hours at $60^{\circ} \mathrm{C}$ and sonicated for one hour to achieve proper mixing. The prepared sol-gels were left for at least 24 hours to allow release of entrapped gasses. 


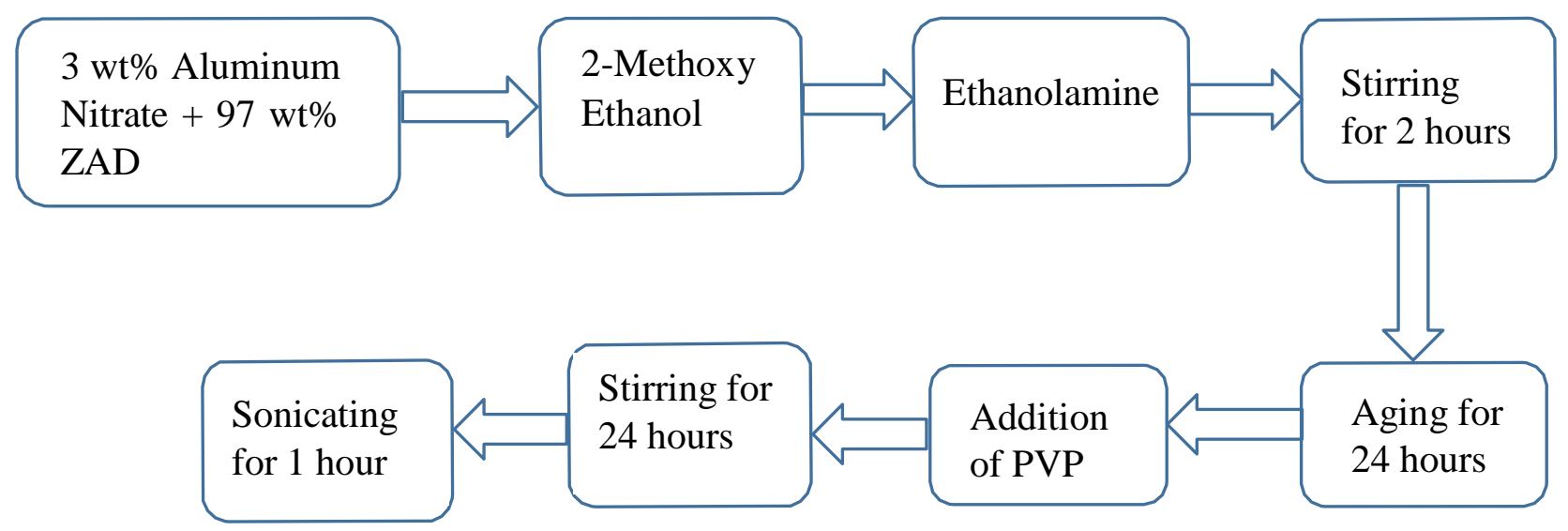

Figure 3.2: Schematic representation of the AZO synthesis

\subsection{Ink characterization}

The prepared inks were characterized by determining their viscosities and contact angles on glass. The thermo-gravimetric analysis of the inks was also carried out to measure the organics degradation behavior during sintering.

Viscosity was determined for each of the inks using a Brooker field DV-II + Pro viscometer. Two to three drops of the inks were placed in the equipment's sample cup and a rotating $52 \mathrm{~mm}$ cone spindle was introduced to cause shearing effect. Shearing rates of 2 to $60 \mathrm{~s}^{-1}$ were used for the AZO ink containing $10 \mathrm{wt} \%$ PVP, 0.4 to $10 \mathrm{~s}^{-1}$ and 0.1 to $2.0 \mathrm{~s}^{-1}$ for the $15 \mathrm{wt} \%$ PVP and $20 \mathrm{wt} \%$ PVP inks respectively. At regular increment of the shearing rates within the range mentioned for each ink, the viscosities were measured.

Static contact angles were measured for AZO inks 1, 2 and 3, while, the advancing and receding angle of contact were further determined for ink 2 . The contact angle measurements were done through the sessile drop method. Static angle of contact was measured by forcing out a drop of the ink from an electronic single channel pipette (Thermo Scientific Matrix). The pipette was 
subsequently pulled away and images of the droplets were taken with a high-resolution camera. For the advancing and receding contact angles, $10 \mathrm{ml}$ of the ink was made to flow out continuously from the pipette tip onto the glass substrate and the deposited ink was subsequently sucked back into the tip. Images and video recordings taken during the deposition and sucking processes were analyzed by using image analysis software and by visual inspection. For all the contact angle measurements, the goniometer tip was positioned at approximately $1 \mathrm{~mm}$ from the glass surface.

Thermal analyses of the AZO inks were carried out on Thermogravimetric analyzer (Perkin Elmer Pyris 1 TGA), which monitors the weight changes over time as the ink is being heated to a high temperature. In doing this, 15 to $20 \mathrm{mg}$ of each ink was placed in a specially designed ceramic container and hung onto the TGA highly sensitive balance. The sample was heated in the TGA furnace to a high temperature of $800^{\circ} \mathrm{C}$ at the rate of $10^{\circ} \mathrm{C}$ per minute and was held at $800^{\circ} \mathrm{C}$ for 15minutes. The weight of the remaining material in the container is measured at regular intervals of time as the temperature increased.

\subsection{Fabrication/DW of AZO Features}

\subsubsection{DW equipment}

A Nordson EFD multi-axis dispensing robot equipped with Nordson EFD Performus V Fluid Dispenser was used for direct writing of features in this work (Figure 3.3). The robot is equipped with an operating table that travels back and forth (x-direction) in a moving range of 300 $\mathrm{mm}$, and a dispensing head that moves both sideways (y-direction) and in the vertical direction ( $\mathrm{z}$ direction). It has a moving range of $320 \mathrm{~mm}$ in the y axis and $100 \mathrm{~mm}$ in the $\mathrm{z}$-axis with $50 \mu \mathrm{m}$ resolution in all directions. Its capability in combining motions in the three axes and circular 
motion, coupled with programmable interface enables deposition of materials in digitally precise locations. It is also capable of printing at speed as high as $800 \mathrm{~mm} / \mathrm{s}$ and can hold up to thirty thousand points in a single printing program routine with 255 programs capacity.

The system is an extrusion type fluid dispensing mechanism and uses air pressure to force materials loaded in an EFD syringe out. Precisely regulating the air pressure in the syringe barrel is very important for a successful printing operation. Thus, the Nordson EFD Performus V fluid dispenser used in conjunction with the robot was employed in achieving the pressure control. The pressure controller has an operating range of 0 to 100 psi with a resolution of 0.5 psi. Its capability for creating vacuum pressure in the syringe barrel also enables handling of low viscosity fluids.

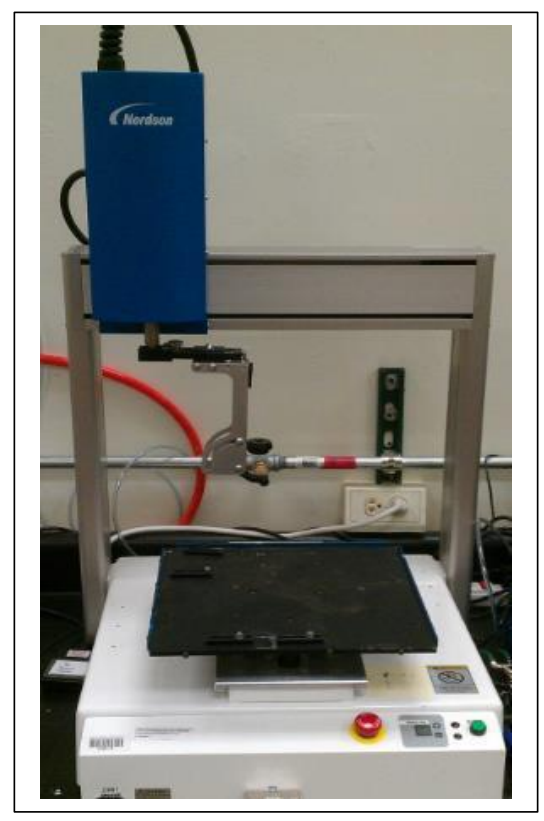

(a)

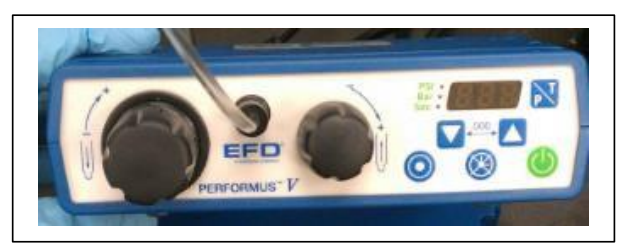

(b)

Figure 3.3: Images of the (a) Nordson Multi-axis robot and (b) Nordson Performus V pressure controller 


\subsubsection{Feature fabrication through DW}

Pattern printing of the AZO was carried out on glass substrates by filling the material into a plastic syringe fitted with a printing tip. The inks were transferred into the syringe by using a pipette or spatula depending on the particular ink viscosity. The syringe was then securely mounted on the fluid dispensing robot head, while the glass on which the pattern was to be printed was secured with clamps on the printing stage. Pressure and writing speed desired for the printing was then input for the actual printing to be done.

Figure 3.4 shows the parameters that affect the properties of printed features. In selecting the printing parameters to be used, a bracketing approach was adopted for defining two extreme cases of printing conditions. The minimum pressure and maximum speed at which the ink with highest viscosity could be printed to form a continuous line while using the smallest nozzle size were determined.

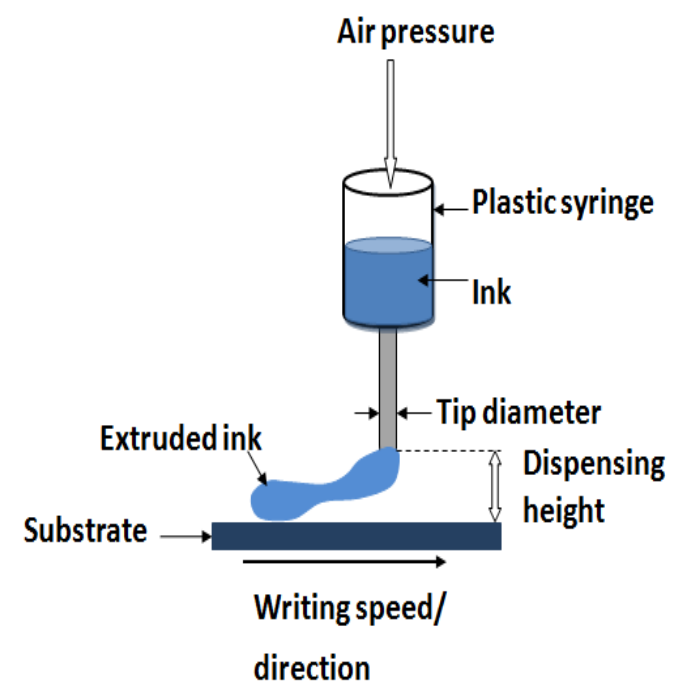

Figure 3.4 Schematic showing the printing parameters 
Also, the lowest writing speed and maximum pressure combination that produced patterns without bulges along the feature length for the lowest viscosity ink while using the largest nozzle size to be considered was determined. Other printing conditions were subsequently selected in the printable region so defined. The dispensing heights (tip-substrate clearance) were selected to be equal to the internal diameter of the nozzles used for the printing in each case.

In order to determine the interactions and the effects of the printing parameters on the properties of the printed patterns, a combination of full and blocked factorial experimental designs were adopted with feature geometry (spread) as the response. Four factors, which include writing speed, pressure, viscosity and tip size were considered in the experimental design. Three levels of each factor were selected within the printable range earlier defined. For the writing speed, 1, 3 and $5 \mathrm{~mm} / \mathrm{s}$ were selected while 10, 15 and 20 psi were picked for the air pressure. The viscosities of the inks used were controlled by the PVP contents and were found to be 2592, 15269 and 57373 cP. Three tip sizes of 100, 150 and $200 \mu \mathrm{m}$ were used for the printing and these values correspond to the dispensing heights used in each case. The dispensing heights were monitored using an optical Dino Capture 2.0 microscope.

At first, the tip size factor was blocked as all other factors, which include viscosity, writing speed, and air pressure were considered at a fixed tip size of $1500 \mu \mathrm{m}$. Thereafter, the experiment was repeated at moderate viscosity value of $15,269 \mathrm{cP}$ while, tip size, air pressure and writing speed were varied within the range earlier mentioned. 


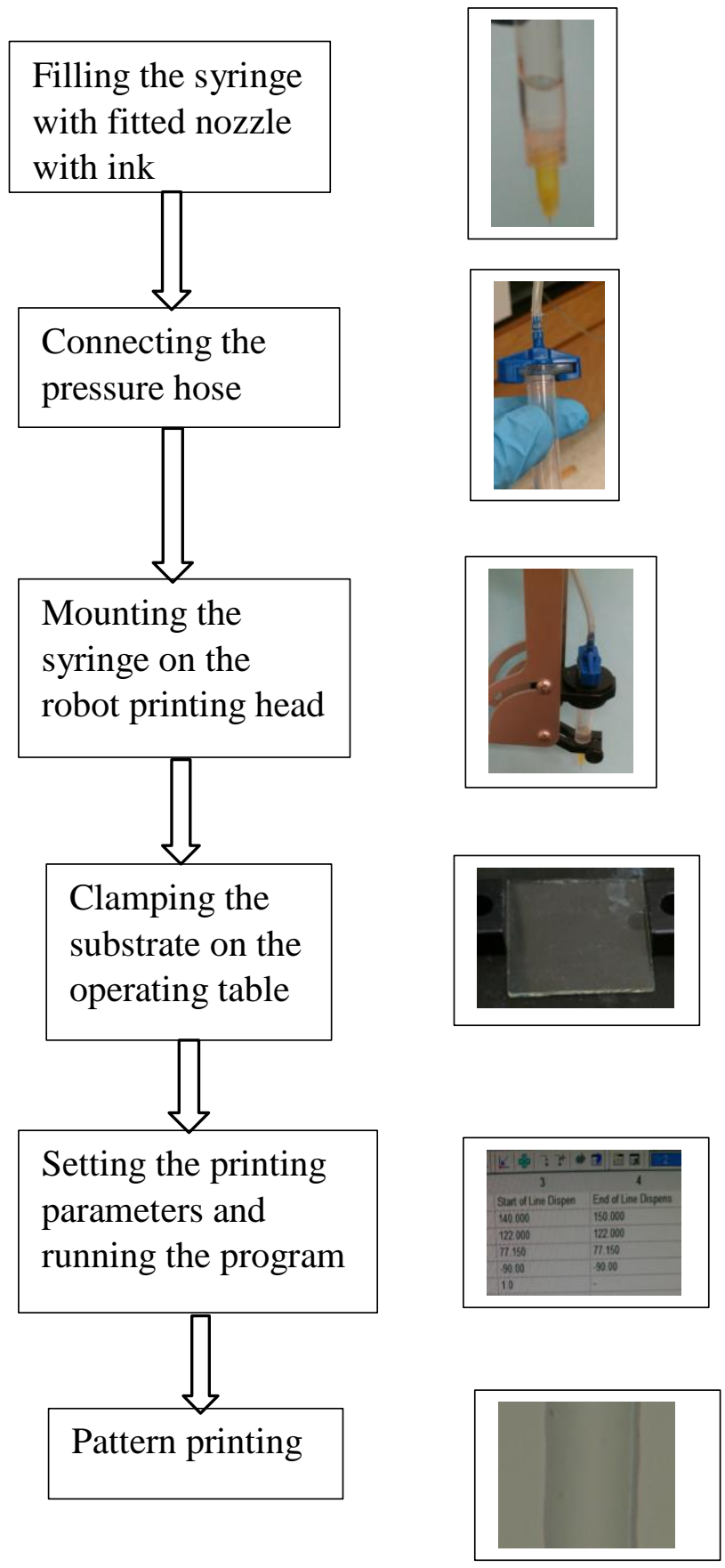

Figure 3.5 Schematic representation of the feature printing process 


\subsection{Interaction and Significance of the Printing Parameters}

Analysis of variance (ANOVA) statistical tool was used to determine the level of interactions among the printing parameters. The significance of the effects of these parameters on the feature geometry, specifically, on the spread of the printed features was also determined. Ten replicates of the spread readings were obtained in each factor-level combination. Thus, a total of two hundred and seventy data points were used in each round of ANOVA. For the factorial experiment with blocked viscosity factor, three levels of each factor: tip size, pressure and writing speed were selected. Also, when tip size was fixed, three levels of the factors viscosity, pressure and writing speed were again considered. Details of the ANOVA parameters for the experimental set up are shown in Table 3.2 below.

The within and between groups variations and effects were determined by calculating the sum of squares (SS) and the mean squares (MS) using equation 3.1(a)-(j). The mean square ratios obtained were compared with the table values corresponding to the degree of freedoms at $95 \%$ confidence level $(\mathrm{p} \leq 0.5)$. Two and three factor interactions among the parameters were also examined, in order to determine their level of significance on the feature geometry.

$$
\begin{aligned}
& \underline{\sum_{\mathrm{c}}^{2}} \underline{\mathrm{T}}^{2} \\
& \mathrm{SS}_{\mathrm{c}}=\operatorname{nrg} \quad \mathrm{N} \\
& \underline{\sum \mathrm{T}_{\mathrm{r}}^{2}}-\underline{\mathrm{T}}^{2} \quad \ldots . \quad \ldots \quad \ldots \quad \text { (b) } \\
& \mathrm{SS}_{\mathrm{r}}=\operatorname{ncg} \mathrm{N} \\
& \underline{\underline{\mathrm{T}_{\mathrm{g}}^{2}}}-\underline{\mathrm{T}^{2}} \quad \ldots . \quad \ldots \quad \ldots \quad \text { (c) } \\
& \mathrm{SS}_{\mathrm{g}}=\mathrm{ncr} \\
& \frac{\sum \mathrm{T}_{2}}{\mathrm{cr}}-\mathrm{T}^{2} \\
& \text { с }-\mathrm{SS}_{\mathrm{r}} \quad \ldots \quad \ldots \quad \ldots \\
& \mathrm{SS}_{\mathrm{cr}}= \\
& \text { ng N }
\end{aligned}
$$


Table 3.2 Factors, levels and the degree of freedoms of experimental parameters

Blocked viscosity factor

\begin{tabular}{|c|c|c|}
\hline Source & Levels & Degree of freedom \\
\hline Tip size & $100,150 \& 200$ & 2 \\
\hline Pressure & $10,15 \& 20$ & 2 \\
\hline Speed & $1,3 \& 5$ & 2 \\
\hline Tip size * pressure & & 4 \\
\hline Tip size * speed & & 4 \\
\hline Pressure*speed & & 4 \\
\hline Tip size * pressure* speed & & 8 \\
\hline Residual & & 20 \\
\hline \multicolumn{3}{|l|}{ Blocked tip size factor } \\
\hline Source & Levels & Degree of freedom \\
\hline Viscosity & Ink 1,2 and 3 & 2 \\
\hline Pressure & $10,15 \& 20$ & 2 \\
\hline Speed & $1,3 \& 5$ & 2 \\
\hline Viscosity $*$ pressure & & 4 \\
\hline Viscosity *speed & & 4 \\
\hline Pressure*speed & & 4 \\
\hline Viscosity * pressure* speed & & 8 \\
\hline Residual & & 20 \\
\hline
\end{tabular}




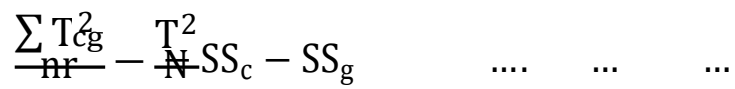

$$
\begin{aligned}
& \mathrm{SS}_{\mathrm{cg}}= \\
& \stackrel{\sum \mathrm{Trg}^{2}}{\mathrm{n}}-\mathrm{T}^{2} \mathrm{SS}_{\mathrm{r}}-\mathrm{SS}_{\mathrm{g}} \quad \ldots \quad \ldots \quad \ldots \\
& \mathrm{SS}_{\mathrm{rg}}=
\end{aligned}
$$

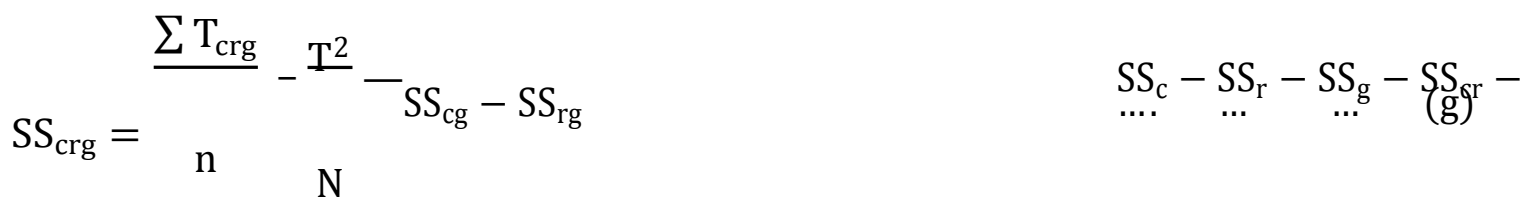

$$
\begin{aligned}
& \mathrm{SS}_{\text {residual }}=\mathrm{SS}_{\text {total }}-\text { all previous SS } \quad \ldots . \quad \ldots \quad \ldots \quad \text { (h) } \\
& \mathrm{MS}=\mathrm{SS} \\
& \text { DF } \\
& \ldots \quad \ldots \quad \ldots \quad \text { (i) } \\
& \text { MS } \\
& \text { MSR }=\text { MS }_{\text {residual }} \quad \cdots \quad \cdots \quad \ldots \quad \text { (j) }
\end{aligned}
$$

where $\mathrm{g}$ is the number of groups (Levels of pressure), $\mathrm{c}$ is the number of columns (Levels of writing speed), $\mathrm{r}$ is the number of rows (Levels of tip size or viscosity), $\mathrm{N}$ is the number of observations or the sample size (27), $\mathrm{n}$ is the number of replications (10), $\mathrm{T}_{\mathrm{c}}$ is the total of each column, $\mathrm{T}_{\mathrm{r}}$ is the total of each row, $\mathrm{T}_{\mathrm{g}}$ is the total of each group, $\mathrm{T}$ is the sum of the all values.

\subsection{Drying and Sintering of Printed Patterns}

All printed features were kept at room temperature and allowed to solidify as they continue with the spreading process. Prior to sintering, the features were further dried on a hot plate (Fisher Scientific Isotemp) at $200^{\circ} \mathrm{C}$ for 10 minutes to burn off the solvent. In order to burn off the polymer and crystallize the metal oxide, all the printed patterns were sintered by heating at $500^{\circ} \mathrm{C}$ in a MPI KSL 1100X lab furnace. A heating rate of $5^{\circ} \mathrm{C} / \mathrm{min}$ was maintained for all the sintering operations 
and a maximum temperature of $500^{\circ} \mathrm{C}$ was considered adequate, as no further weight loss was recorded in the TGA results beyond this temperature for all the inks. The sintering temperature of $500^{\circ} \mathrm{C}$ was maintained for 2 hours to allow for complete crystallization. The materials were allowed to cool down to room temperature in the furnace before being removed.

\subsection{Characterization of Printed Features}

The printed patterns were characterized through various methods. The geometrical, morphological, microstructural and crystallographic properties of the written features as affected by the printing parameters were all determined. Also, the electrical conductivity of the features with and without UV excitation was measured.

\subsubsection{Geometrical characterization}

The fabricated features were characterized geometrically before and after sintering by acquiring their optical images using a Leica optical microscope, magnification 10, 20, 50X, equipped with a frame grabber (Guppy, Allied Vision Technology). Typical images of the written feature and tip used are shown in Figure 3.6. The widths of the deposited patterns were measured using image-processing software (ImageJ NIH). Width measurements were taken at equally spaced positions along the length of the written feature, in order to get a good idea of the feature

uniformity. Feature spreads, S, taken to be the percentage of the feature width in excess of the tip size used for the printing were determined by Equation 3.2 given below.

$$
\begin{aligned}
& S=\frac{w-t_{i}}{x} \\
& \begin{array}{llllll}
\times 100 \% & \ldots & \ldots & \ldots & 3.2
\end{array}
\end{aligned}
$$


where $\mathrm{w}$ is the measured width of the written feature and $t_{i}$ is the internal diameter of the tip used for the printing.
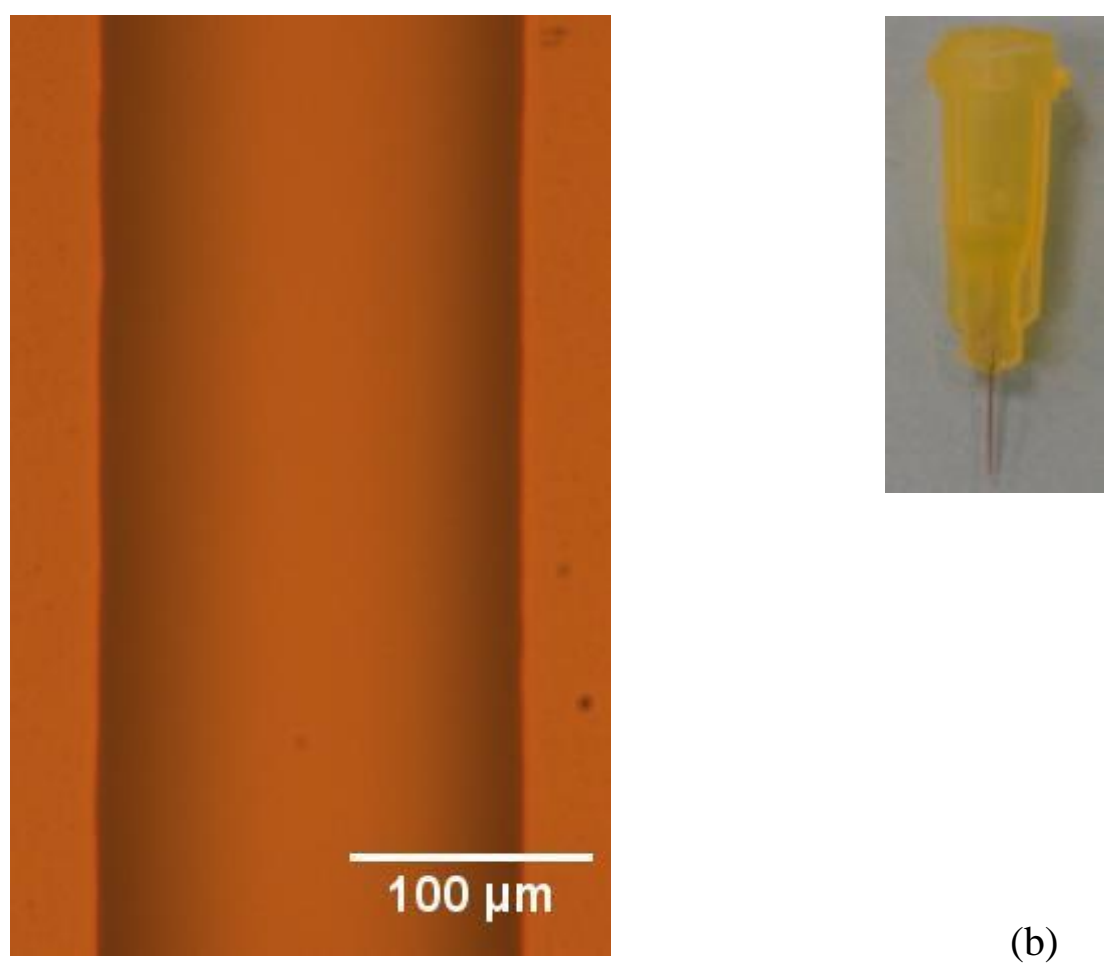

(a)

(b)

Figure 3.6 (a) Optical images of a feature printed with $100 \mu \mathrm{m}$ tip, 20 psi pressure, $100 \mu \mathrm{m}$ dispensing height and $3 \mathrm{~mm} / \mathrm{s}$ writing speed using ink 2 and (b) digital image of a $100 \mu \mathrm{m}$ tip used for printing

The heights and shapes of the features were obtained by using a stylus profilometer (Tencor Alpha-Step 200). The measured height and width were used in estimating the aspect ratio, r, given by Equation 3.3. This gives an indication of the feature's roundness.

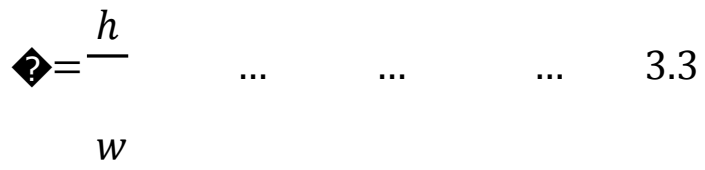

Where $\mathrm{h}$ is the measured height of the feature in $\mu \mathrm{m}$ and $\mathrm{w}$ is the feature's measured width in $\mu \mathrm{m}$. 


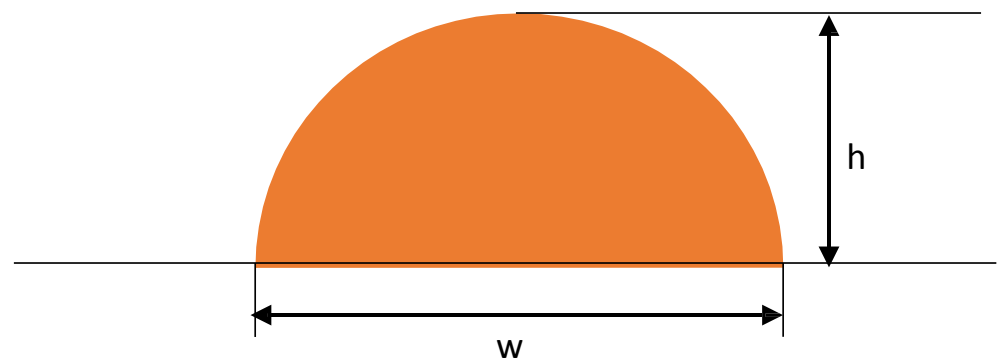

Figure 3.7 Schematic representation of a printed feature's cross sectional view

\subsubsection{SEM}

$\mathrm{SEM}$ is a non-destructive characterization technique used to generate high-resolution images and acquire chemical composition of materials. SEM can produce very high-resolution images of a surface, revealing details less than $1 \mathrm{~nm}$ in size. Focused beam of high-energy electrons is used to scan the sample surface and electron interactions with atoms in the material generate a variety of signals at the surface of solid specimens. The derived signals, which can be detected and measured, are used to deduce numerous material properties, ranging from surface topography and composition to phase orientation. The beam current absorbed by the specimen can also be detected and used to create images of the distribution of specimen current [72].

An inelastic scattering resulting from electron-atom interaction leads to secondary electron being ejected from the k-shell of the specimen. Since the secondary electrons are of low energy $(<50 \mathrm{eV})$, they originate within a few nanometers of the sample surface. When these electrons are detected, measured, and reconstructed, it gives an image of the surface it originated from. Also, characteristic X-rays are emitted when the electron beam removes inner shell electron from the sample, causing a higher-energy electron to fill the shell and release energy. The characteristic X- 
rays of different elements are separated into energy spectrum by an energy-dispersive (EDS) detector and EDX system software is used to analyze the energy spectrum in order to determine the composition and measure the abundance of specific elements.

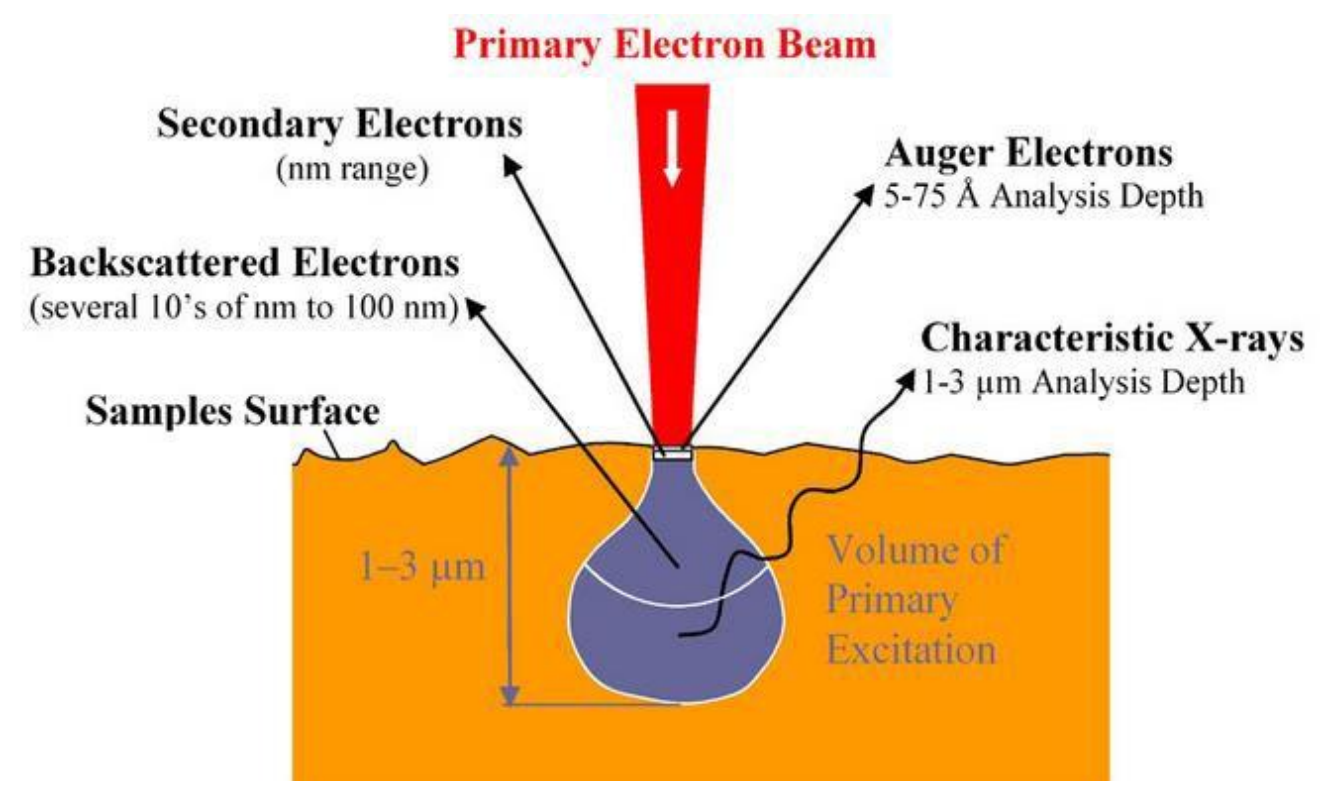

Figure 3.8 Schematics showing SEM operating principle

The imaging in this work was carried out using SEM-Hitachi S-4700F, which works in secondary electron mode. Prior to SEM examination, the samples were sputtered with Au-Pd to form a $10 \mathrm{~nm}$ thick film using a Denton Desk V Sputtering machine. The sputtering is to improve the sample's conductivity and prevent charging. The electron gun was powered with a voltage of $5 \mathrm{KV}$ and $10 \mathrm{~mA}$ current for the imaging. A working distance of $12 \mathrm{~mm}$ was maintained in the ultra-high resolution mode and necessary adjustments were made to bring the object to focus. The images were subsequently taken. Electron dispersive X-ray (EDX) analysis to obtain the elemental composition of the material was performed using an INCA X-ray analysis system. The data were acquired at $20 \mathrm{KV}$ and $10 \mathrm{~mA}$ current in the UHRA mode. The high energy used is to obtain good 
probe depth into the material. Expert analysis feature of the EDX software was then used to identify elements corresponding to the peaks found on the spectrum.

\subsubsection{XRD}

$\mathrm{XRD}$ is another non-destructive and powerful laboratory based technique used for revealing and characterizing the crystal structures of substances in several states. It can also be used to determine phase composition and preferred orientation as well as determine microstructural properties of materials. Lattice parameters, grain size, epitaxy, thickness of thin films and multilayers, and atomic arrangement are also features that can be determined through $\mathrm{XRD}$. XRD is based on constructive interference of monochromatic $\mathrm{x}$-rays and a crystalline material. The X-rays generated from $\mathrm{Cu}$ or $\mathrm{Co}$ source in a cathode ray tube is filtered to produce monochromatic radiation, collimated to concentrate using grating devises and directed towards the sample.

Interaction of x-ray photons and electrons in atoms, in form of collision, often results in some of the photons from the incident beam being deflected away from the original travelling direction. For a situation where no energy is lost and the wavelength of the scattered x-rays did not change due to the collision (elastic or Thompson scattering, only momentum transferred), the diffracted photons are then detected, processed and counted, as these scattered x-rays carry information about the electron distribution in the material.

However, production of constructive interference (and a diffracted ray) from the interaction of the incident rays (x-ray photons) with the sample is achieved when Braggs Law (Equation 3.4) 
is satisfied. The law relates the wavelength of electromagnetic radiation to the diffraction angle and the lattice spacing in a crystalline sample.

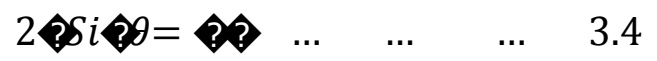

where $\mathrm{d}$ is the spacing between atomic layers, $\lambda$ is the wavelength of the $\mathrm{x}$-ray, $\theta$ is the scattering angle, and $\mathrm{n}$ is an integer representing the order of the diffraction peak.
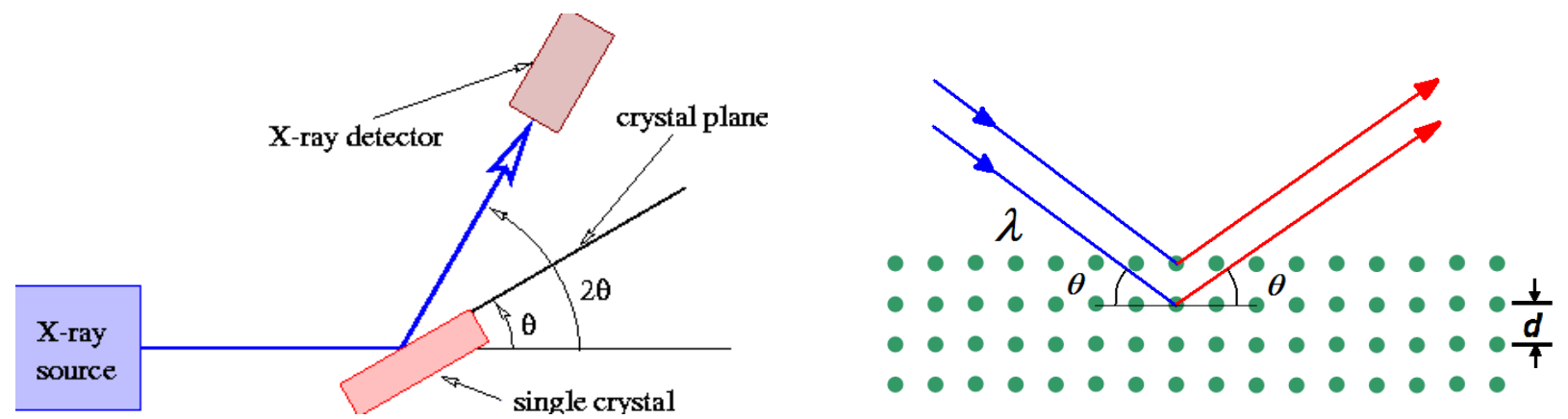

Figure 3.9 Schematics depicting the principle of XRD

Therefore, by scanning the sample through a range of $2 \theta$ angles, all possible diffraction directions of the lattice are attained due to random orientation of the material. If the atoms are arranged in a periodic fashion, as in crystals, the diffracted waves will consist of sharp interference maxima (peaks) with the same symmetry as in the distribution of atoms. Conversion of the diffraction peaks into d-spacings allow the identification of the mineral because each mineral has a set of unique d-spacings. Typically, this is achieved by comparing the d-spacings with standard reference patterns. Measuring the diffraction pattern therefore allows us to deduce the distribution of atoms in a material.

The XRD analysis of the samples was conducted using a PANalytical X'Pert Pro X-ray Diffractometer. A generator operating voltage of $45 \mathrm{kV}$ and $40 \mathrm{~mA}$ current with copper used as 
the anode material. A spinning sample stage and gonio-scanning axis with a goniometer radius of $240 \mathrm{~mm}$ were used. The sample surface was continuously scanned through an angle (20) of 20 to 80 degrees in steps of $0.03^{\circ}$ and a scan time of 100 seconds per step were used. The divergence slit was fixed at $0.5^{\circ}$ and a $100 \mathrm{~mm}$ distance maintained between focus and divergence slit. The analysis was carried out at room temperature and the obtained spectra were analyzed using Highscore Plus analysis software.

Crystalline phases and crystal orientation of the materials printed with different printing parameters were deduced from the obtained spectra. The peak positions, peak widths, and peak intensities obtained from the spectra were used in estimating the grain sizes and the lattice constants of the printed materials. Average grain size of the materials was calculated using Scherrer's formula (Equation 3.5) and the lattice constants were calculated by using Brag's law (Equation 3.6). An estimation of the state of residual stresses was also obtained by comparing the obtained peak positions to the standard $\mathrm{ZnO}$ peak positions obtainable in the JCPDS cards.

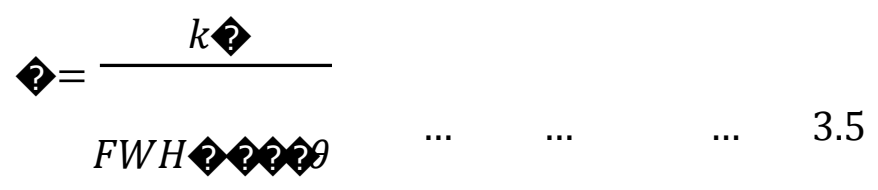

where $\mathrm{k}$ is the Scherer constant, $\lambda$ is the $\mathrm{X}$-ray wavelength of the incident $\mathrm{K} \alpha$ radiation given as $0.154056 \mathrm{~nm}, \mathrm{FWHM}$ is the full width at half maximum of the respective peak and $\theta$ is the diffraction peak angle.

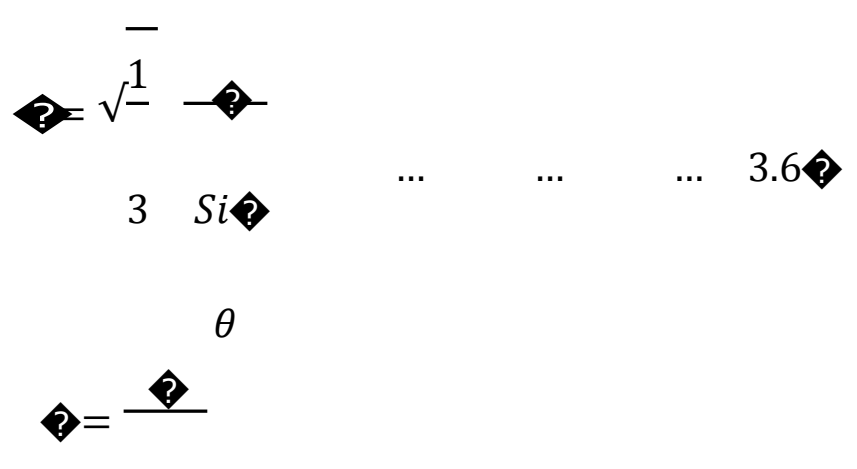


$\begin{array}{llll}\ldots & \ldots & \ldots & 3.6\end{array}$ 
where $\lambda$ is the $\mathrm{X}$-ray wavelength of the incident $\mathrm{K} \alpha$ radiation given as $.154056 \mathrm{~nm}$ and $\theta$ is the diffraction peak angle.

\subsubsection{Electrical characterization}

Terminals were created on the samples by depositing silver paste on the two ends of the AZO feature to form a metal-semiconductor-metal (MSM) configuration as shown in Figure 3.10. An approximate length of one millimeter was maintained for the semiconductor part of the MSM setup. After deposition of the silver paste, the setup was dried on a hot plate at $200^{\circ} \mathrm{C}$ for fifteen minutes to ensure creation of good contact at semiconductor-metal interface. The ohmic nature of the contact created was confirmed by measuring current-voltage (I-V) relationship of the MSM as a bias voltage of -5 to $5 \mathrm{~V}$ was applied. An Agilent 4155C semiconductor analyzer was used for the I-V measurement. All the samples selected for the electrical characterization were made into the MSM configuration as earlier explained. The I-V characteristics of the AZO features were then measured at room and temperatures of $25,50,75$ and $100{ }^{\circ} \mathrm{C}$.

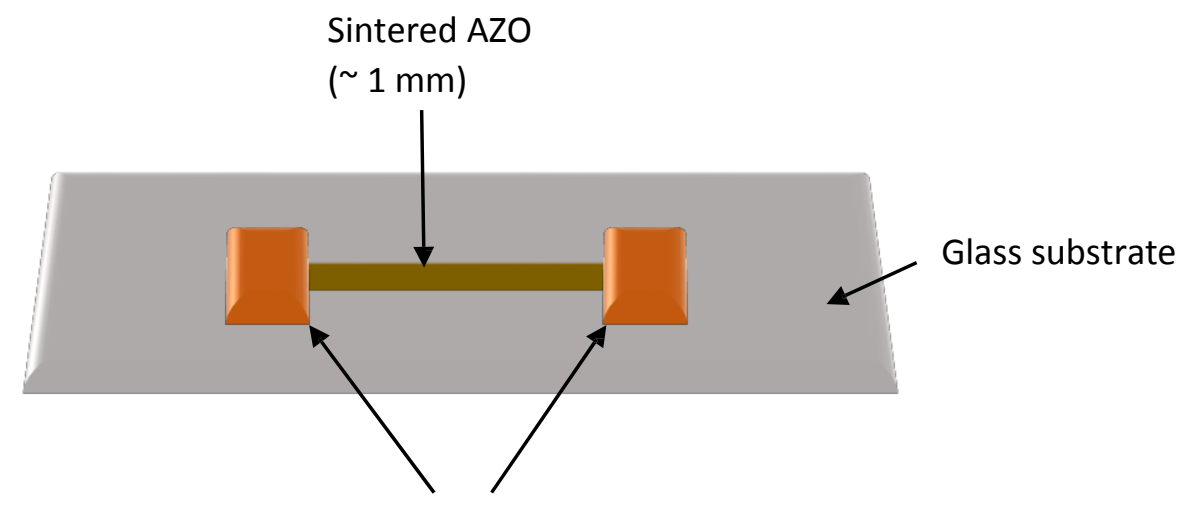

Silver terminals

Figure 3.10 Schematic of the MSM device 
The photoconductivity of the MSM devices in the presence of UV light was also investigated at room temperature. The I-V characteristics of the MSM was measured using the same Agilent 4155A semiconductor analyzer mentioned earlier, as the device was being illuminated with $365 \mathrm{~nm}$ wavelength UV rays placed at about $5 \mathrm{~mm}$ from the device. The surface of the device was illuminated for 10 minutes to allow stable I-V relationship. The UV source was powered by connecting it to an Agilent 3611A voltage source. Three input voltages to 4.5 and $6 \mathrm{~V}$ were used to vary the irradiance of the UV rays on the device surface. The peak values, which corresponds to the minimum resistance in each case was determined.

Lastly, the photocurrent response to UV light was investigated by switching on and off the UV light at intervals of three minutes as current flowing through the device at constant voltage of $5 \mathrm{~V}$ is being measured. To determine the activation and relaxation time, the UV light as left on for 30 minutes while current is measure at constant $5 \mathrm{~V}$ and subsequently switched off the decline in current over a 30 minutes period was obtained. A schematic of the procedure followed is shown in figure 3.11 .

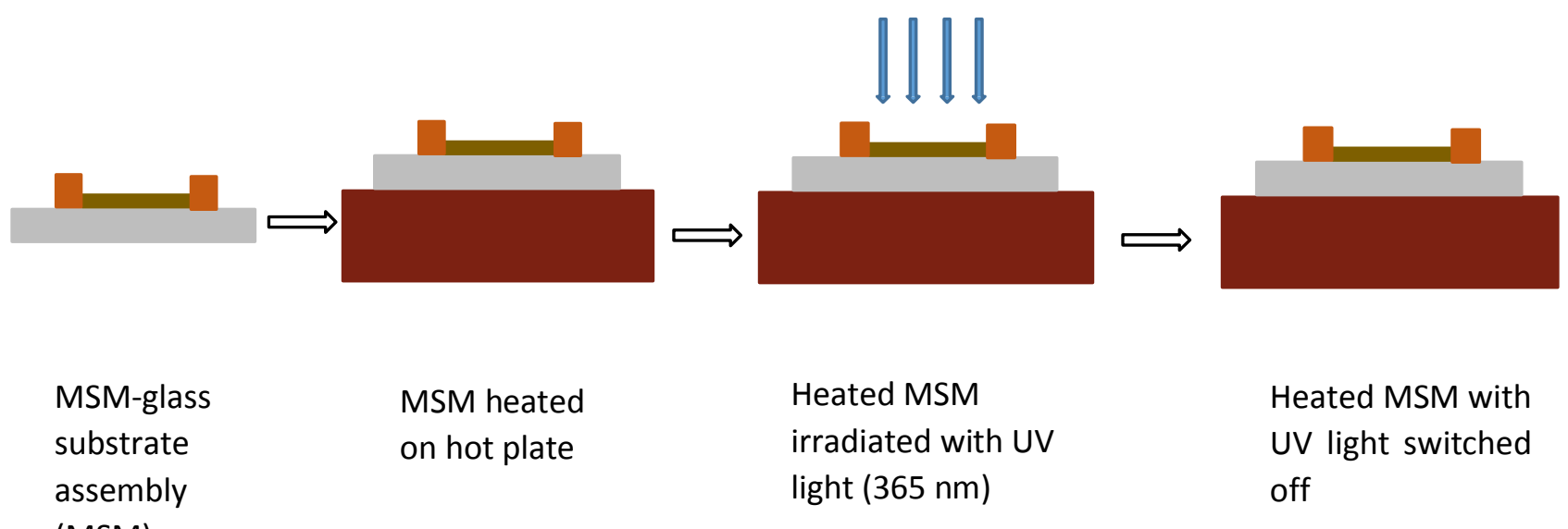

(MSM)

Figure 3.11 Schematic representation of the electrical characterization process 


\section{DW OF AZO INKS: INVESTIGATION OF PRINTING PARAMETERS}

Geometrical consistency of features deposited through NBDW is an essential requirement for potential applications of patterned microstructures. The properties of these patterned features, geometry inclusive, are however determined by some factors. These printing factors can be broadly classified into two, which are: material (ink) parameters and machine or process factors. It is therefore required that the ink is characterized to establish the material factors pertinent to the printing before selecting the machine parameters.

\subsection{Ink Characterization}

Of great importance among the material properties that influence the geometry and resolution of printed features are the viscosity [30] and surface energy of the ink [73]. Therefore, the viscosities and the contact angles formed by the inks on cleaned glass substrate were measured. Ink viscosity which has been reported [15] to play major role in determining the selection of subsequent printing parameters was determined by using a Brookerfield DV-II + Pro viscometer. The viscosities of the inks used for printing in this study were tuned by the PVP contents of 10,15 and $20 \mathrm{wt} \%$ added to the prepared solution. Viscosities versus shear rates of the three inks used in this study are shown in Figure 4.1.

Ink 1 has an average viscosity of $2592.4 \mathrm{cP}$ (2.592 Pa.s), whereas ink 2 has a viscosity of 15262. 8 (15.262 Pa.s) and ink 3's viscosity is equal to $57373.18 \mathrm{cP}$ (57.373 Pa.s). Increasing the PVP content from 10 to $15 \mathrm{wt} \%$ caused the ink's viscosity to significantly increase by almost seven fold. Also, further increase of the PVP content to $20 \mathrm{wt} \%$ increases the viscosity by almost 20 times compared to the $10 \mathrm{wt} \%$ PVP ink. Figure 4.2 shows the plot of viscosity change with PVP contents, depicting an exponential increase in viscosity as PVP content increases. 


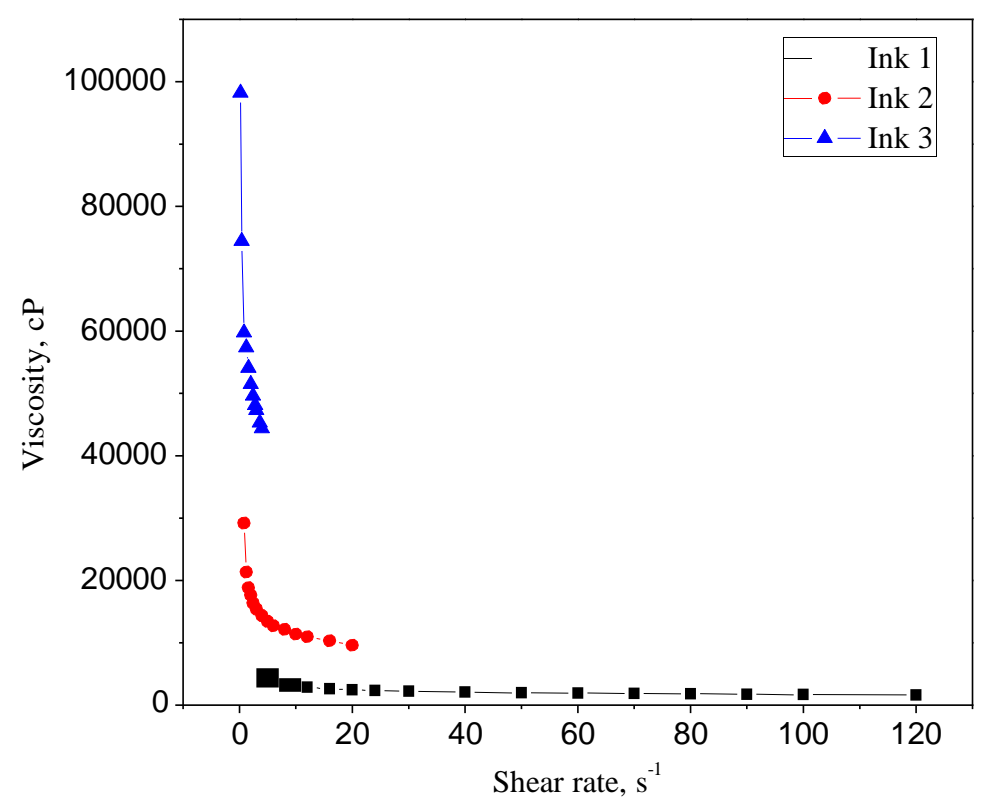

Figure 4.1 Viscosity of the three inks versus shear rate

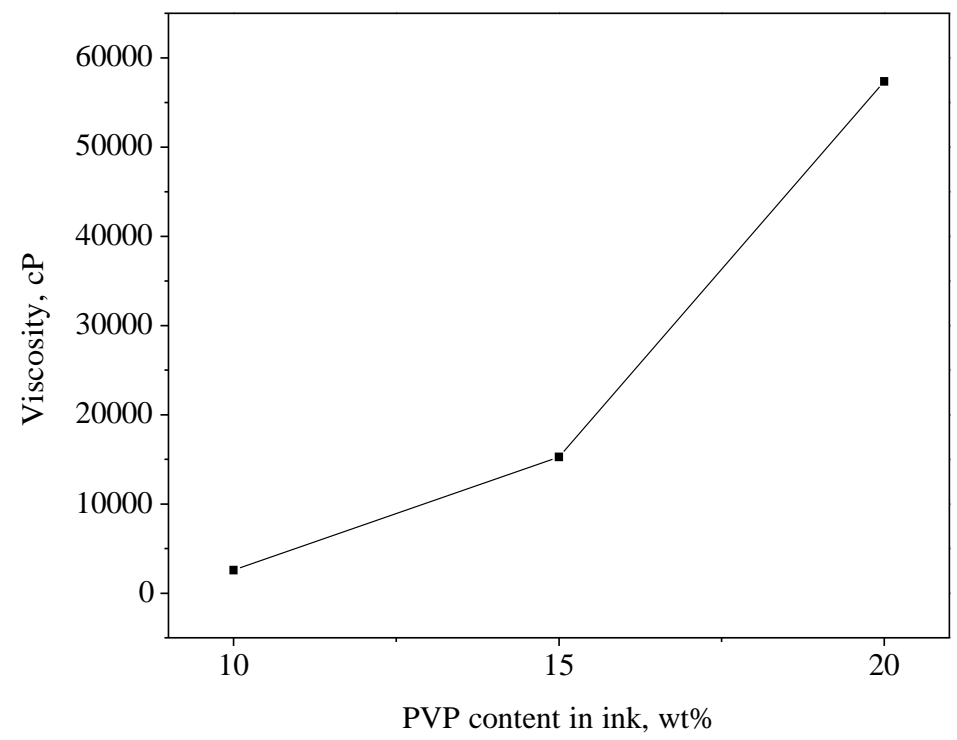

Figure 4.2 Change in viscosity for inks with different PVP contents

Also, individual plots of the inks' viscosities versus shear rate shown in figurers 4.3 to 4.5 show that the inks exhibit non-Newtonian behavior, as the viscosities are found to be changing 
differently for various share rates, portraying a shear thinning behavior. For ink 1 shown in figure 4.3, maximum viscosity of $4965 \mathrm{cP}$ and minimum viscosity of $1649 \mathrm{cP}$ were obtained at shear rates of 4 and $120 \mathrm{~s}^{-1} 1$ respectively. Shear rates of 0.8 and $20 \mathrm{~s}^{-1}$ produce the maximum and minimum viscosities of $29202 \mathrm{cP}$ and $9595 \mathrm{cP}$ respectively for ink 2 (Figure 4.4). Ink 3, plotted in figure 4.5, yields its maximum and minimum viscosities of 8228 and $44401 \mathrm{cP}$ at shear rates of 0.2 and $4 \mathrm{~s}^{-1} 1$, respectively.

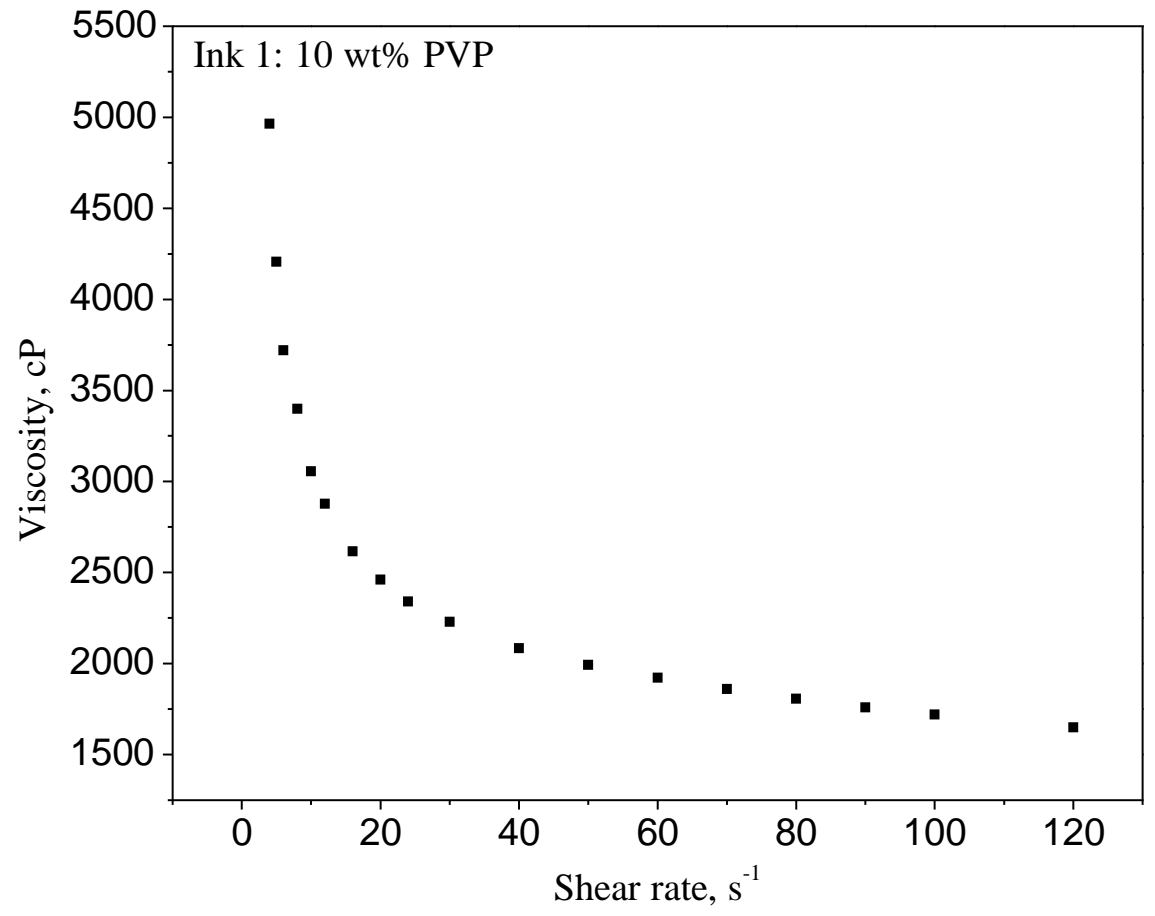

Figure 4.3 Viscosity of ink $1(10 \mathrm{wt} \% \mathrm{PVP})$ at different shear rates 


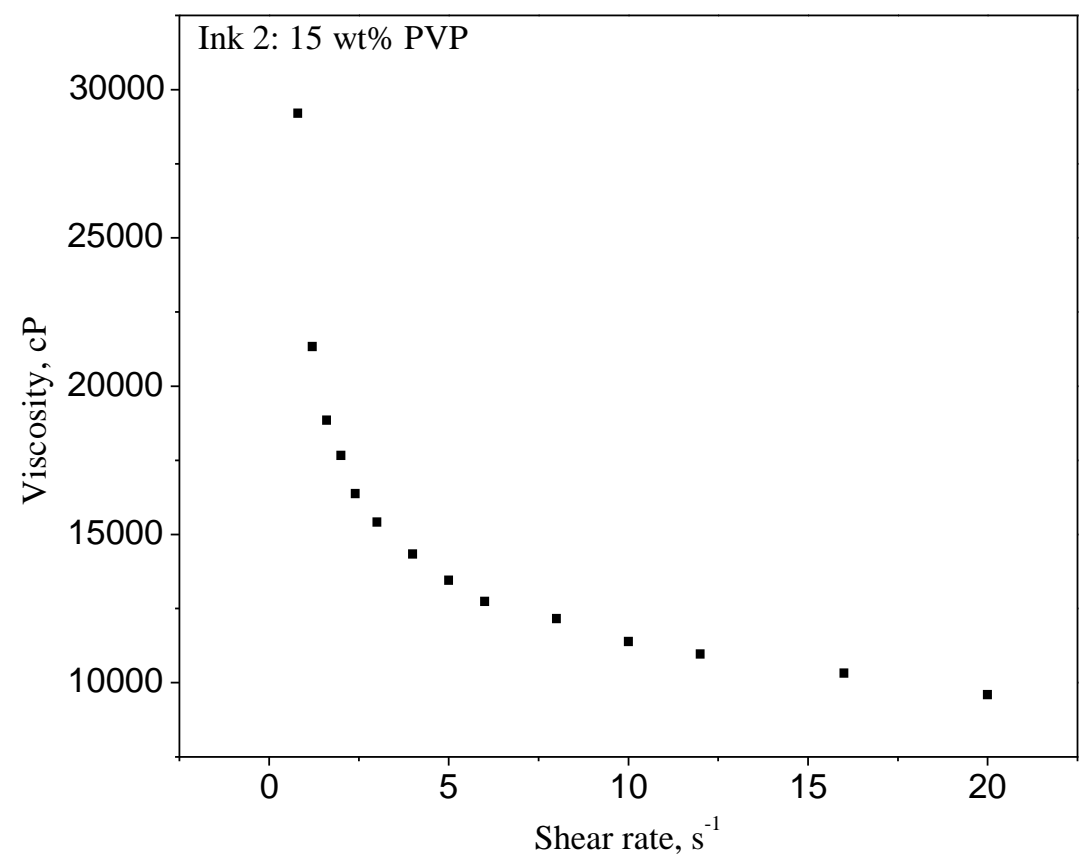

Figure 4.4 Viscosity of ink 2 (15 wt\% PVP) at different shear rate

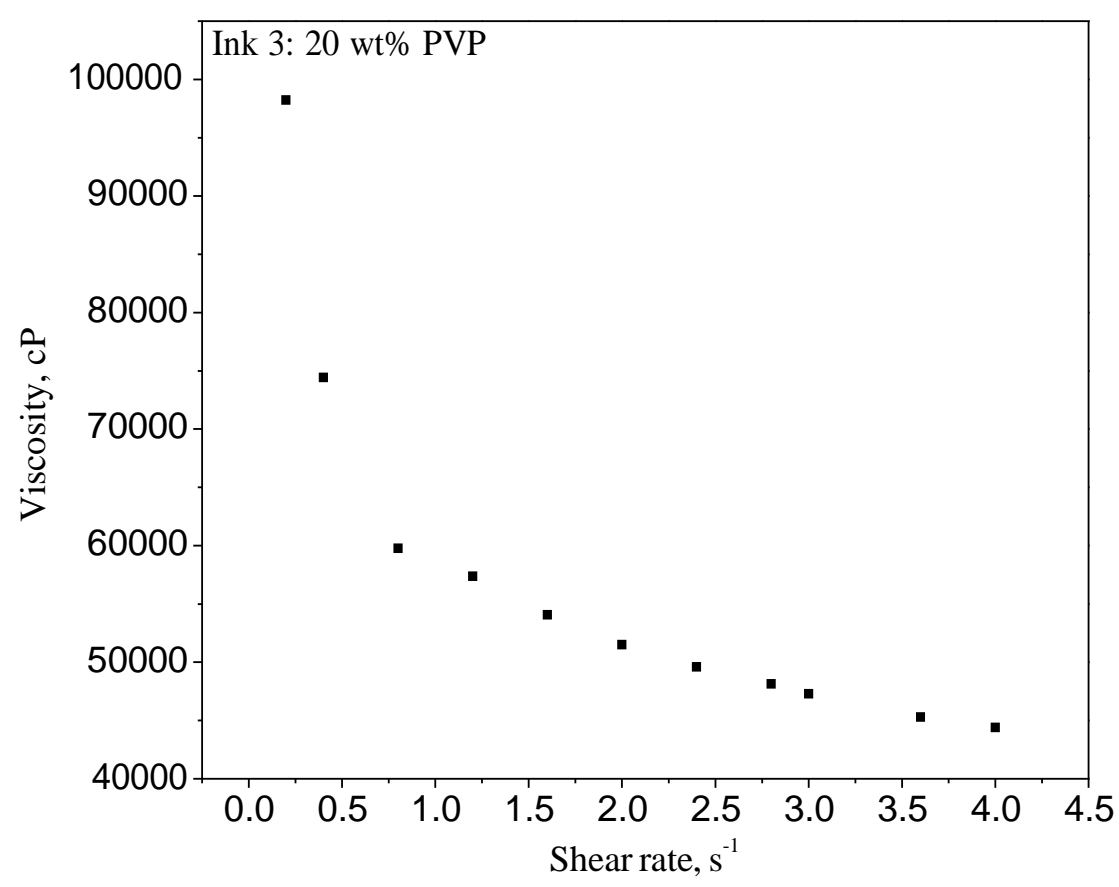

Figure 4.5 Viscosity of ink 3 (20 wt\% PVP) at different shear rate 
Surface energies of both substrate and ink (surface tension) is another important material property that affects the geometry and resolution of printed features. It determines the wettability of the substrate surface by the printing ink. The surface energy is related to the contact angle formed between the substrate and the ink by balancing the interfacial tensions at the three phase contact of solid-liquid and gas as described by Young's equation (Equation 4.1)[74]

$$
\begin{array}{lllll} 
& =\gamma_{s}+\gamma_{l}
\end{array}
$$

Where sis the solid-gas interfacial energy, $\gamma_{s}$ is the solid-liquid interfacial energy, and the $\gamma_{l}$ represents the liquid-gas interfacial energy (i.e. the surface tension) and $\theta$ is the angle between and $\gamma_{s}$ and represents the contact angle between the ink and the substrate surface.

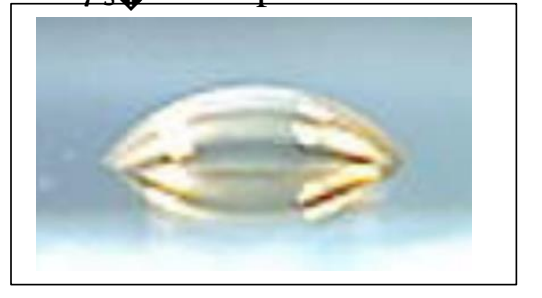

$10 \mathrm{wt} \%$ PVP

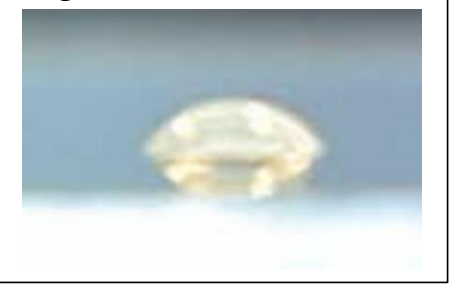

$15 \mathrm{wt} \%$ PVP

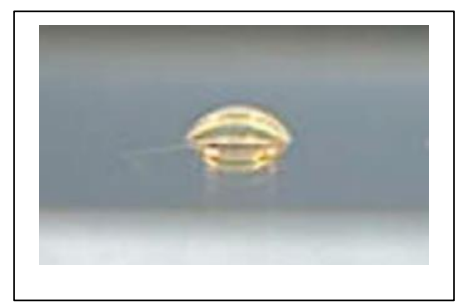

$20 \mathrm{wt} \%$ PVP

Figure 4.6 Contact angle measurement of AZO inks with PVP content

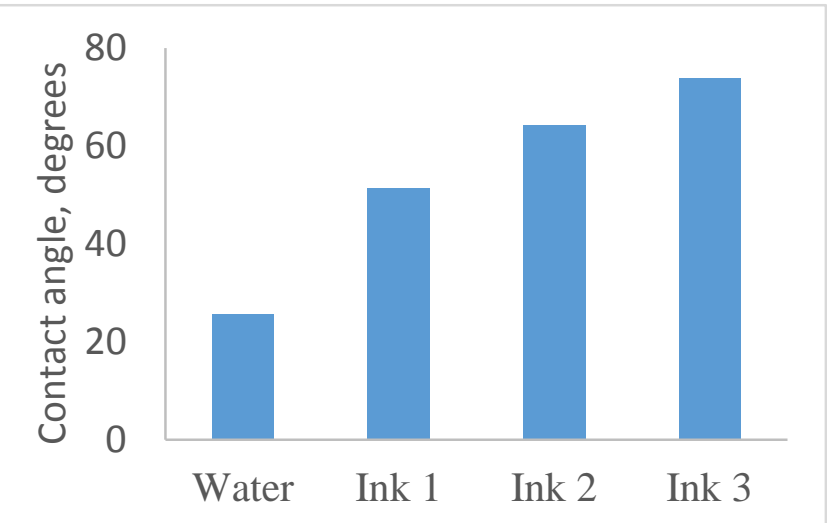

Figure 4.7 Contact angle of deionized water and the three AZO inks on the cleaned glass substrates' surface 
Therefore, wettability can be inferred by measuring the contact angles of the inks on the substrate of interest. The higher the contact angle, the lower the wettability of the substrate surface by the ink. The contact angle of the three inks used in this study as determined by the sessile drop method (Figure 4.6) is found to be $51.6^{\circ}, 64.2^{\circ}$ and $73.4^{\circ}$ for inks with 10,15 and 20 wt\% PVP respectively. It is found that the contact angle increases as the PVP content of the ink increased from 10 to $20 \mathrm{wt} \%$ (Figure 4.7). This is an expected behavior, because the addition of PVP serves as binder to hold the ink molecules together. Although, the inks exhibit varying angle of contacts with the substrate, they all have values less than $90^{\circ}$, which signifies hydrophilic behavior [75].

\subsection{Effect of Printing Parameters}

All the printing parameters, those related to the material properties and process, combine to determine the properties of printed features. In order to determine the influence of the printing parameters on the geometry and the resolution of the printed features, three levels of tip size, air

pressure, writing speed and viscosities tuned by PVP addition were examined. The dispensing height was fixed to be equal to the inner diameter of any particular tip size being used. The spread of the deposited features, estimated as the percentage difference between the width of the printed feature and the inner diameter of the tip size used for the printing.

The influence of dispensing height on the printed pattern is an important consideration and its careful selection is required for reliable printing. For instance, tip writing at distance lower than the height of the feature, which is approximately equal to the internal diameter of the tip at the exit point, will lead to a groove being created on the pattern. Also, the consequences of having the tip farther away from the substrate, than the inner diameter of the tip, ranges from printing of features 
with irregular widths to printing discontinuous patterns and ultimately to non-printing at all. Hence, while printing with 100, 150 and $200 \mu \mathrm{m}$ internal diameter tips, 100, 150 and $200 \mu \mathrm{m}$ were selected as dispensing heights. The use of these dispensing heights in this work resulted after a series of preliminary experimenting with various dispensing heights ranging from 100-300 $\mu \mathrm{m}$ for the selected tip sizes. Typical optical images of patterns printed at various heights from the substrate are shown in figure 4.8 .

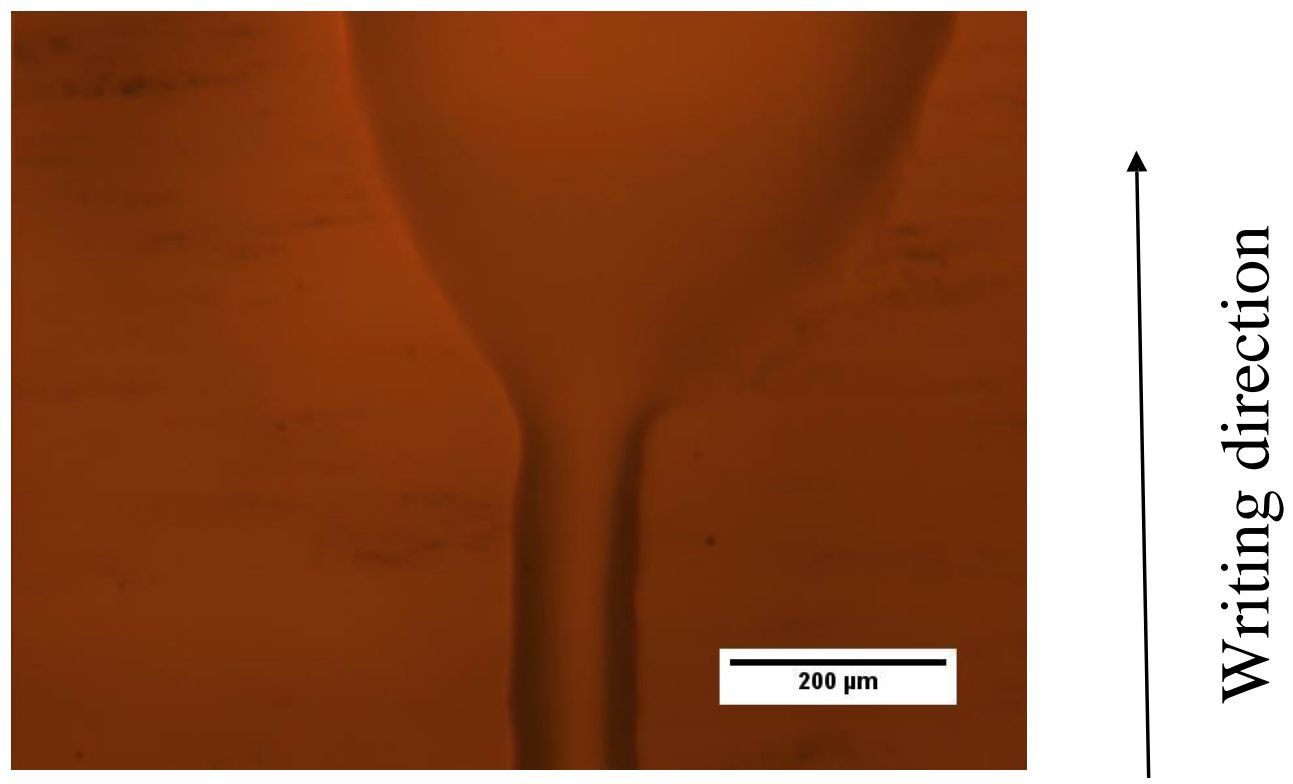

Figure 4.8 Typical image of patterns drawn at high dispensing height

The ink viscosity is an important material property that affects the nature of deposited patterns. Low viscosity is required to permit material extrusion from the nozzle without much difficulty, thus eliminating the need for expensive, high pressure printing equipment. However, inks with relatively high viscosity are needed for printing, in order to prevent the deposited patterns from spreading excessively when in contact with the substrate and thereby improving resolution. 
Low viscosity ink, when exiting the nozzle, spreads significantly before it solidifies thereby exhibiting a very low feature aspect ratio. On the other hand, high viscosity ink may result in nozzle clogging if not properly handled. It is therefore imperative that ink's viscosity is controlled and optimized before successful printing can be achieved.

All the inks were printed with the same printing parameters using $150 \mu \mathrm{m}$ tip size. The choice of the $150-\mu \mathrm{m}$ tip was motivated by the need to use the same printing pressure and speed for the three inks. Figure 4.9 to 4.11 show typical plots of the spread versus inks, characterized by their viscosities, printed at various pressures (Figures 4.9 and 4.10), and writing speeds (Figure 4.11) using a $150 \mu \mathrm{m}$ nozzle. All plots show a decrease in spread of the printed pattern as the viscosity is increased. This behavior occurs irrespective of the writing speed and pressure used. Deviation from linearity is observed in the relationship between spread and ink viscosity at higher pressure and approaches linearity as pressure decreases. Also, it is observed that the low viscosity ink 1 shows high sensitivity to changes in pressure, as changes in pressure result in marked difference in spread. The approximately $420 \%$ spread recorded at 10 psi for pattern printed at 1 $\mathrm{mm} / \mathrm{s}$ increased to $1000 \%$ at 20 psi. Ink 2 shows moderate variation in spread, with changes in pressure at the same writing speed of $1 \mathrm{~mm} / \mathrm{s}$ having only a difference of $60 \%$ is observed, while, only slight difference is observed for ink 3. Changes in speed however, shows an effect on the spread-viscosity relationship, as noticeable differences occur in spread for all the inks. The high spread observed in low viscosity inks can be attributed to the weak intermolecular forces holding the molecules of the ink together, thereby, yielding to the force of gravity and adhesion acting between the substrate and the ink. Also, the high wettability tendency of the ink owing to the low angle of contact observed can be responsible for this. 


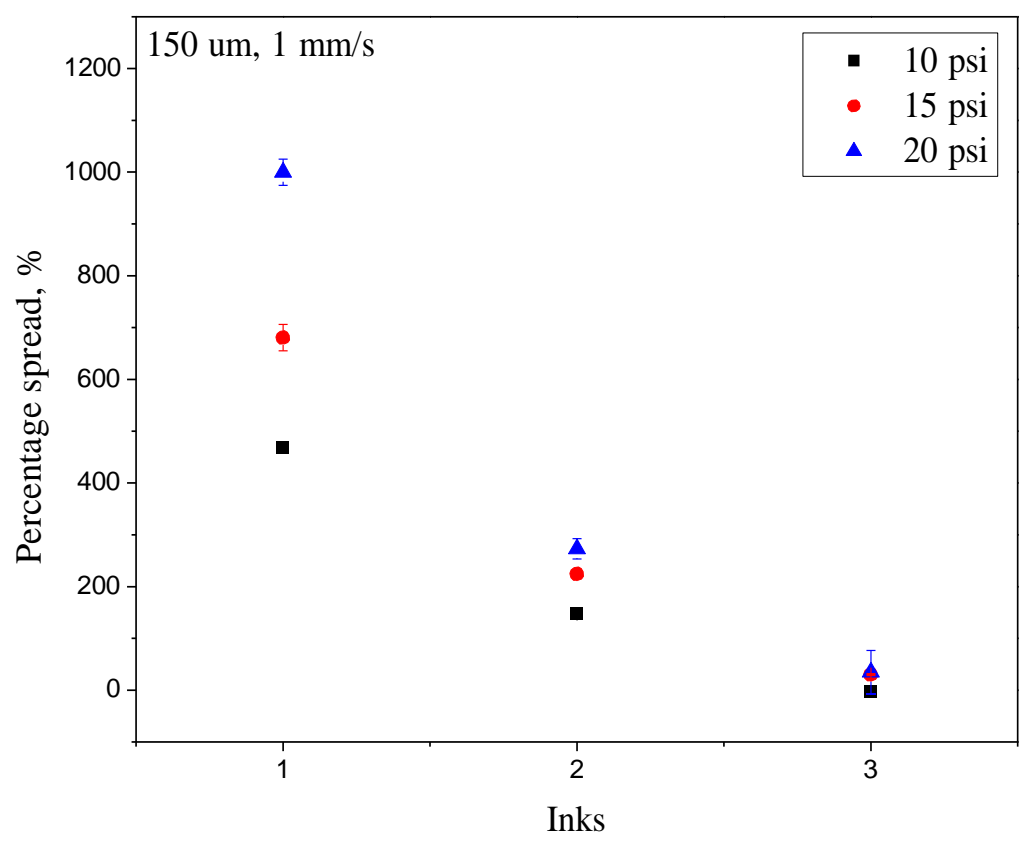

Figure 4.9 Spread for feature printed with different inks and pressures at $1 \mathrm{~mm} / \mathrm{s}$ writing speed, using $150 \mu \mathrm{m}$ nozzle and $150 \mu \mathrm{m}$ dispensing height

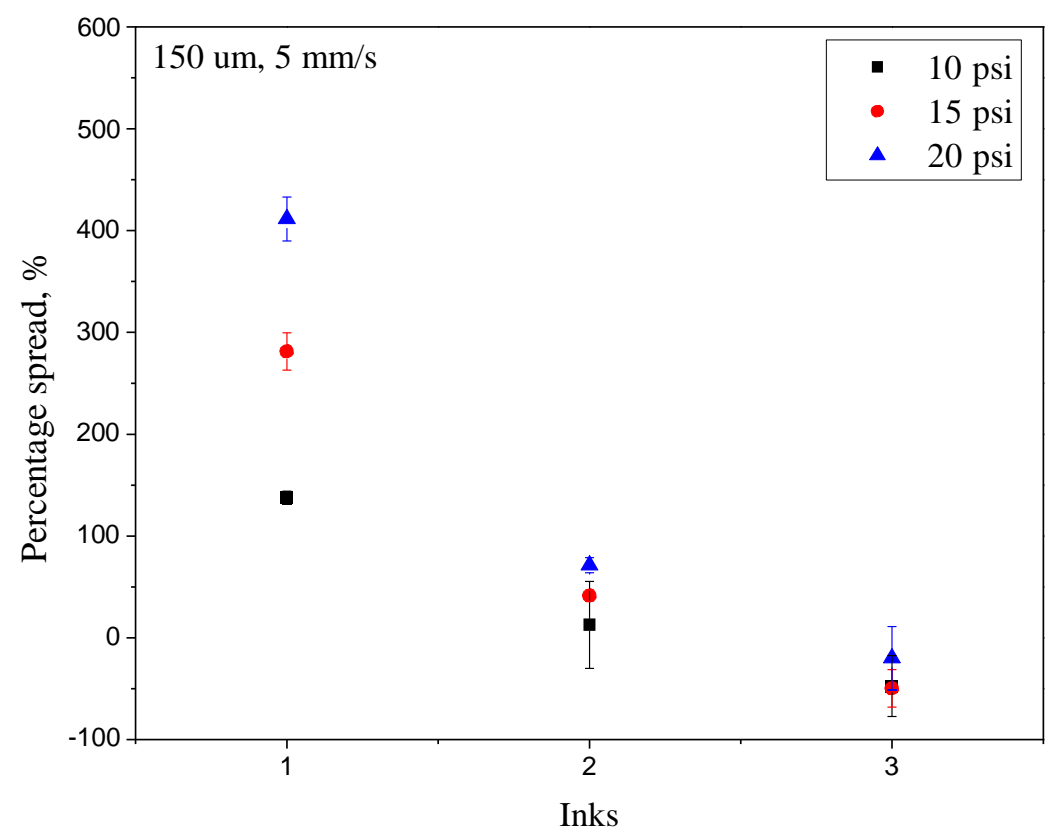

Figure 4.10 Spread for feature printed with different inks and pressures at $5 \mathrm{~mm} / \mathrm{s}$ writing speed, using $150 \mu \mathrm{m}$ nozzle and $150 \mu \mathrm{m}$ dispensing height 


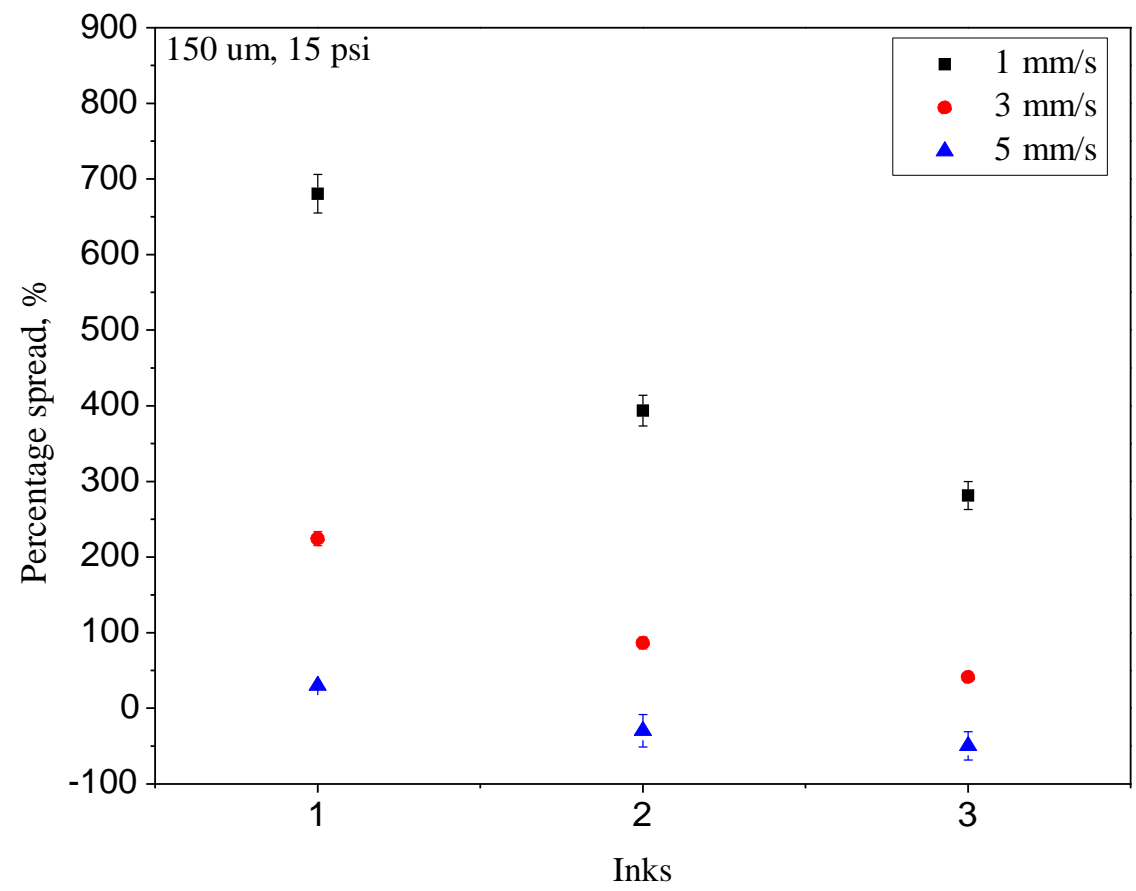

Figure 4.11 Spread for feature printed with different inks and writing speeds at 15 psi, using $150 \mu \mathrm{m}$ nozzle and $150 \mu \mathrm{m}$ dispensing height

The tip provides the channel through which the material is forced out onto the substrate. The size of the tip being used has a direct impact on the quantity of ink to be deposited and consequently on the properties of the deposited features. The effect of the tip size on the properties of the system can extend beyond the physical geometry to the microstructural arrangement of the crystallites. Discussing about the geometry of the printed patterns while printing with ink 2 , the spread increases almost linearly with increasing tip size for all printing pressures and speeds. Figures 4.12 to 4.14 show plots of the spread versus tip sizes at different pressures and speeds. The linear relationship observed between spread and tip size is observed to deviate from linearity at 10 psi while printing ink 2 at $1 \mathrm{~mm} / \mathrm{s}$. Also, printing at large tip size of $200 \mu \mathrm{m}$ results in the spread becoming more sensitive to speed. 


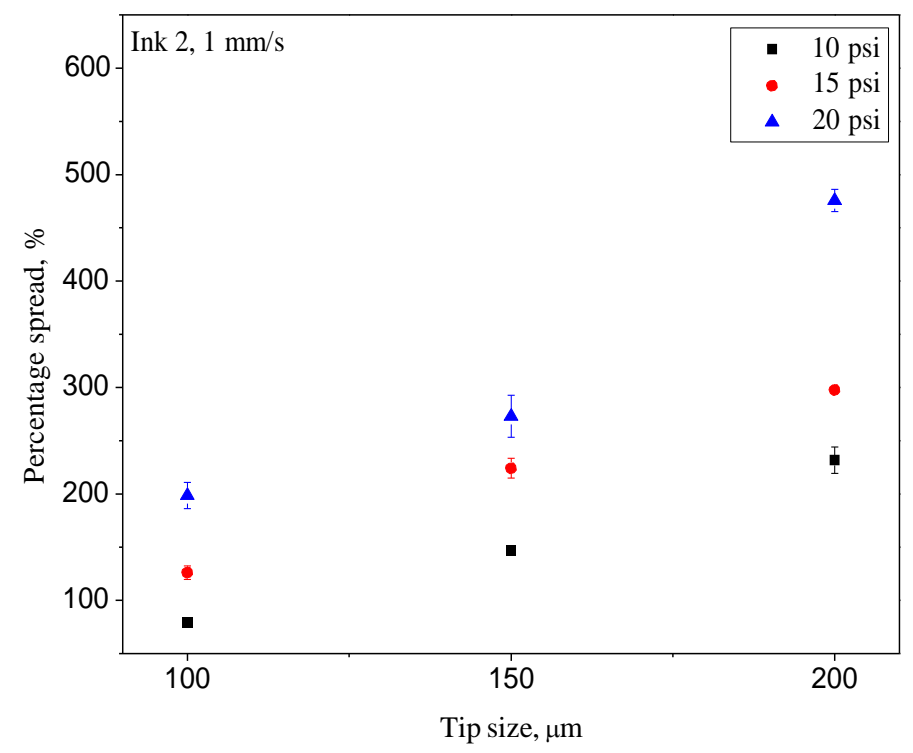

Figure 4.12 Spread for feature printed with different tip sizes and pressures at $1 \mathrm{~mm} / \mathrm{s}$ writing speed, using ink 2 (15 wt\% PVP) and same dispensing heights as the tip sizes

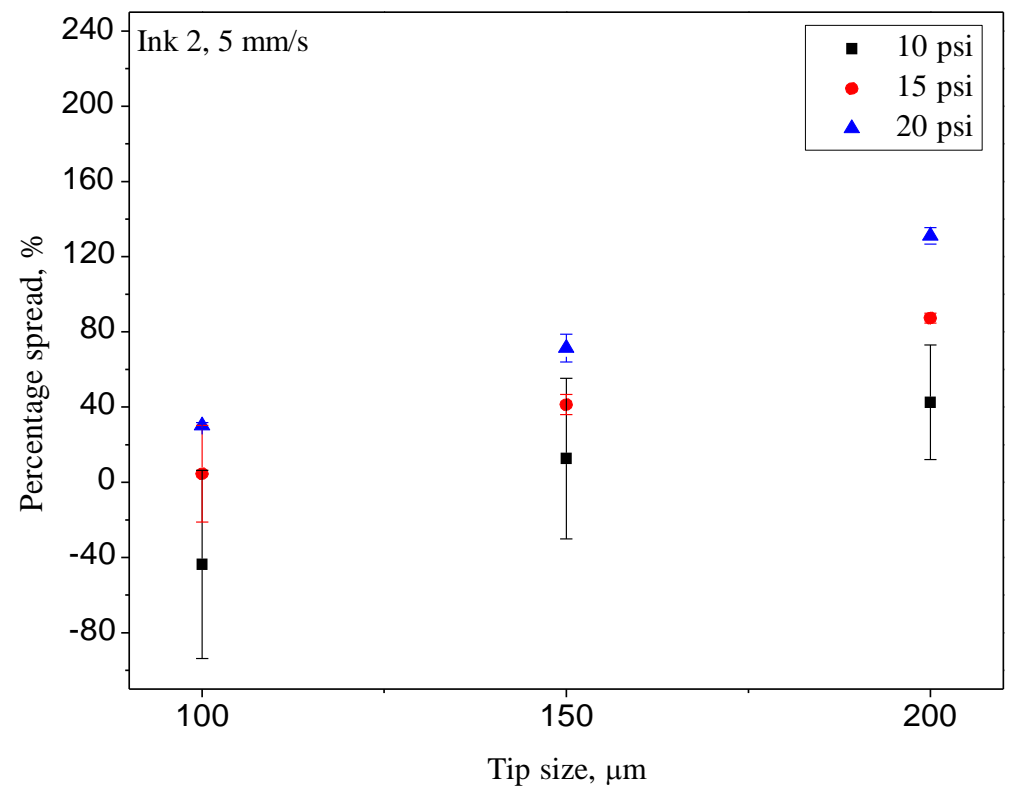

Figure 4.13 Spread for feature printed with different tip sizes and pressures at $5 \mathrm{~mm} / \mathrm{s}$ writing speed, using ink 2 (15 wt\% PVP) and same dispensing heights as the tip sizes 


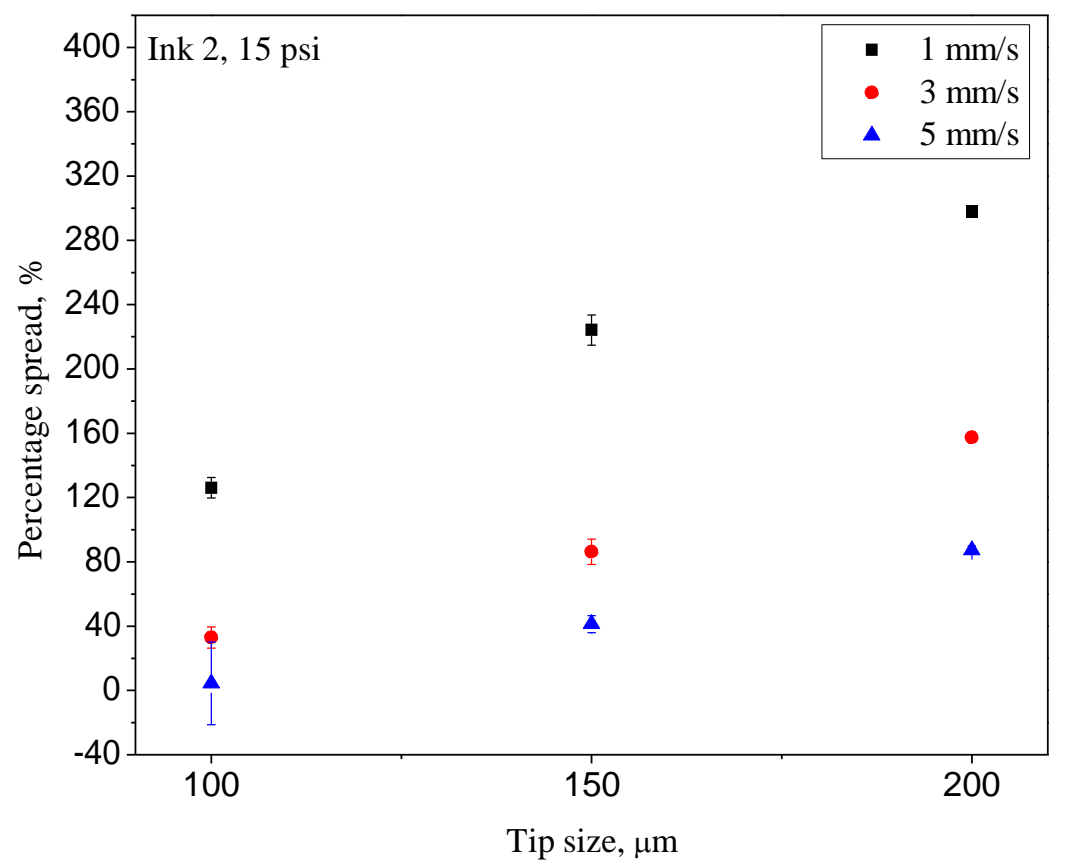

Figure 4.14 Spread for feature printed with different tip sizes and writing speeds at 15 psi, using ink 2 (15 wt\% PVP) and same dispensing heights as the tip sizes

Extrusion pressure required to force out materials from the reservoir is also of great importance in the printing process, as it contributes to the volumetric flow rate of the ink. Pressure optimization is required for a successful printing operation and it is often established based on the viscosity of the ink and tip size to be used. Printing with too high pressure may lead to excessive spreading of the features, while, too low pressure may result in printing of discontinuous features. Figures 4.15 to 4.17 shows the plots of spread versus pressure for ink with different viscosities printed at 1,3 and $5 \mathrm{~mm} / \mathrm{s}$ using $150 \mu \mathrm{m}$ nozzle. A direct proportionality relationship is observed to exist between spread of the written patterns and extrusion air pressure. For all ink viscosities, the spread increases as the pressure increases, with the lower viscosity ink (ink 1) increasing at a higher rate. This is expected since increased applied pressure increases the volumetric flow rate of the ink, thus, increasing the quantity of the deposited material. 


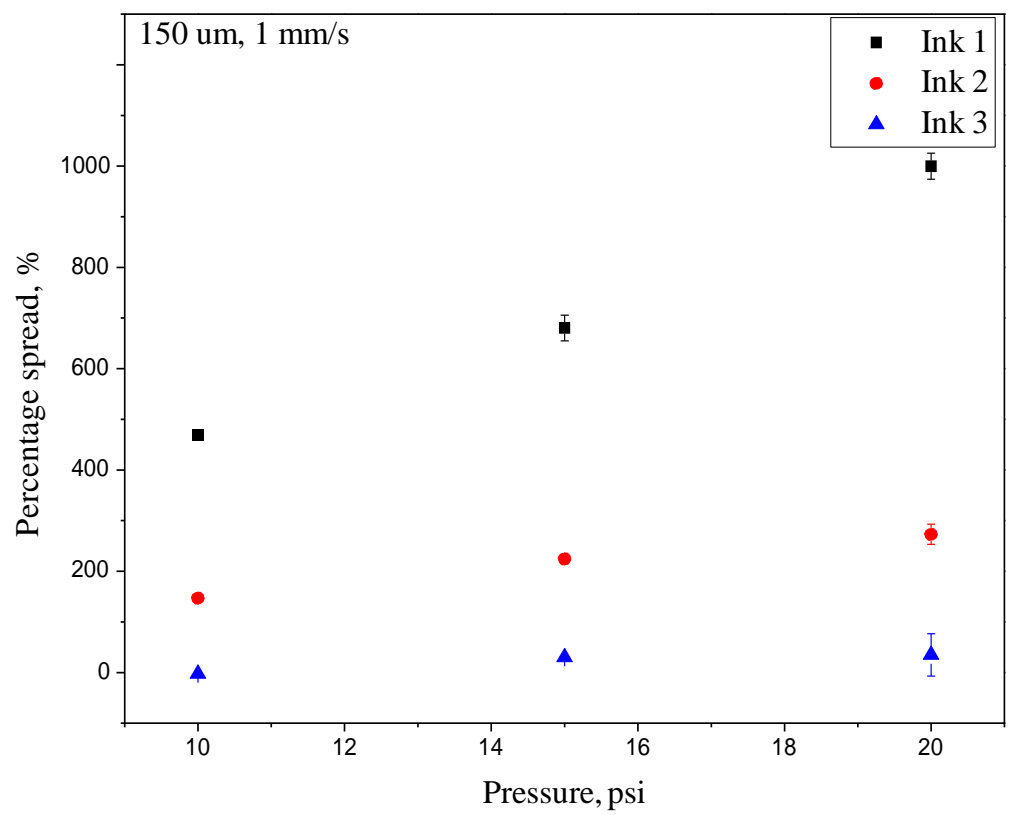

Figure 4.15 Spread versus pressure for features printed with different inks at $1 \mathrm{~mm} / \mathrm{s}$ using $150 \mu \mathrm{m}$ nozzle and $150 \mu \mathrm{m}$ dispensing heights.

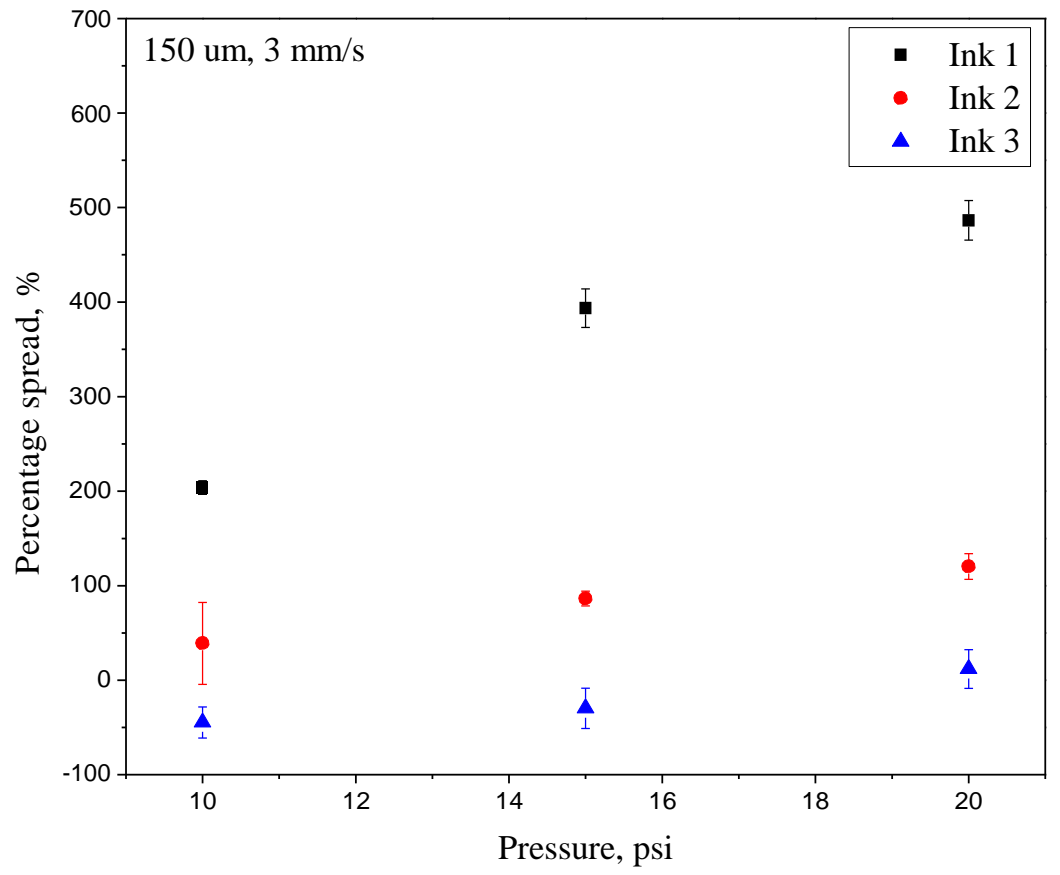

Figure 4.16 Spread versus pressure for features printed with different inks at $1 \mathrm{~mm} / \mathrm{s}$ using $150 \mu \mathrm{m}$ nozzle and $150 \mu \mathrm{m}$ dispensing heights 


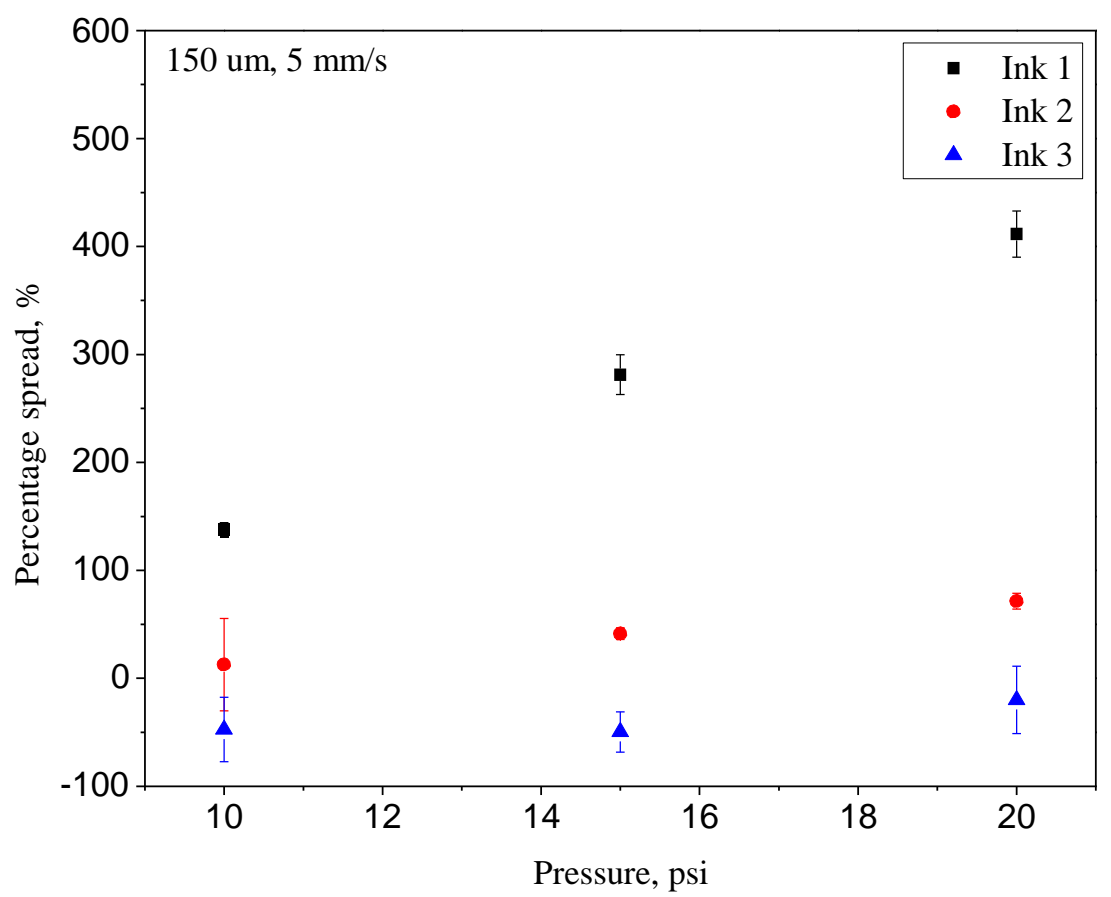

Figure 4.17 Spread versus pressure for features printed with different inks at $1 \mathrm{~mm} / \mathrm{s}$ using $150 \mu \mathrm{m}$ nozzle and $150 \mu \mathrm{m}$ dispensing heights.

The same trend is also observed when spread is plotted against pressure for different tip sizes and fixed viscosity (Figures 4.18 and 4.20). The plots show increase in spread as pressure increases for all tip sizes and the increase was observed to be linear in all cases. However, the linear spread-pressure relationship has higher slope for larger tip size of $200 \mu \mathrm{m}$ while printing at a low speed of $1 \mathrm{~mm} / \mathrm{s}$. Also, while printing at a fast speed, low pressure tends to produce patterns with irregular widths (Figure 4.19) for all tip sizes as depicted by the large standard deviations. This is often the case when the pressure being applied is not enough to pump out material that can keep pace with the speed of writing. 


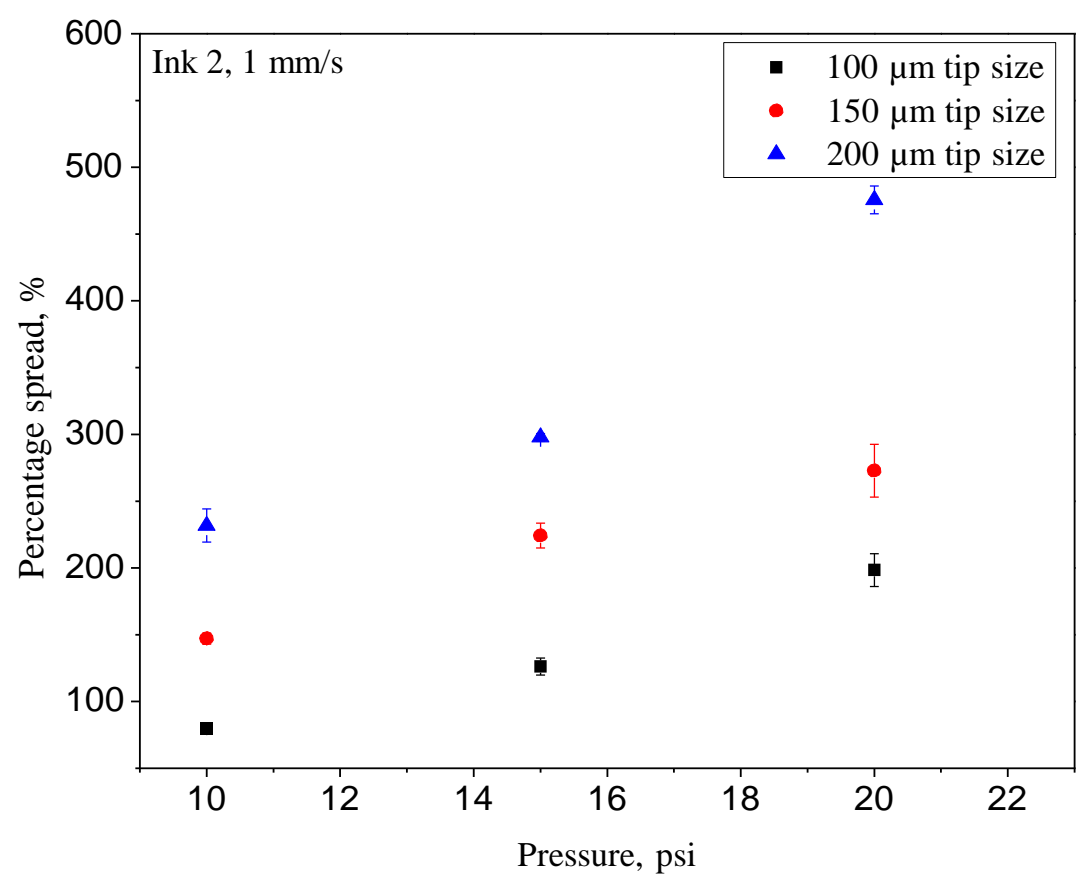

Figure 4.18 Spread versus pressure for features printed with different tip sizes at $1 \mathrm{~mm} / \mathrm{s}$ using ink 2 and same dispensing heights as the tip sizes

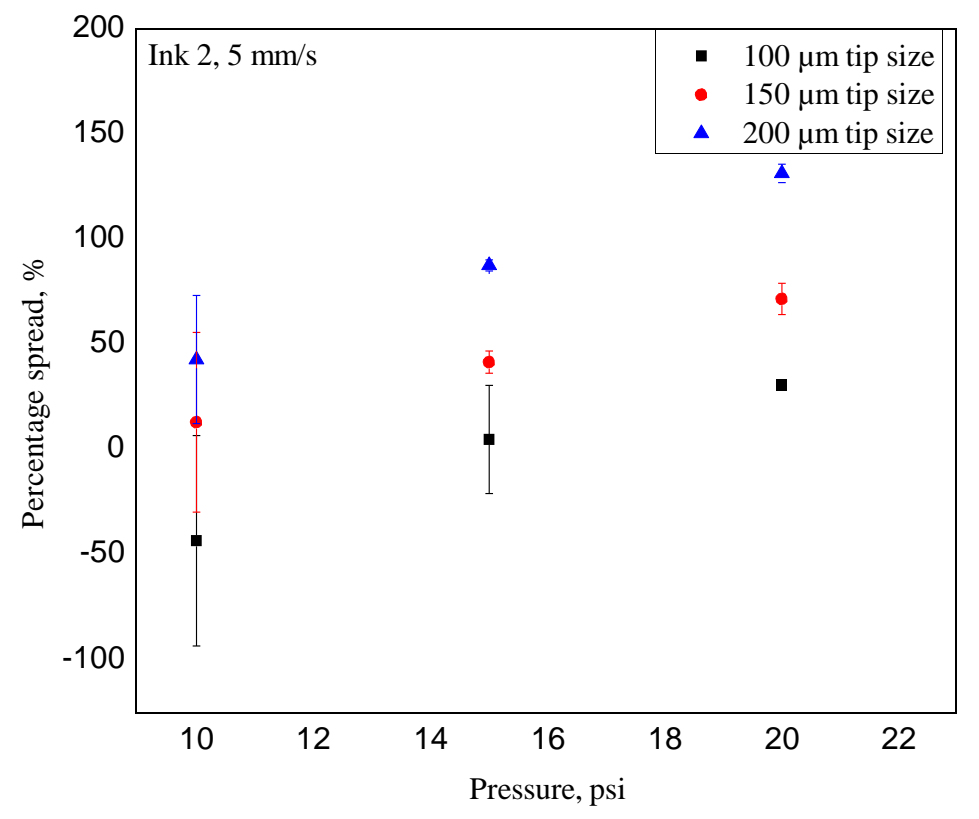

Figure 4.19 Spread versus pressure for features printed with different tip sizes at $5 \mathrm{~mm} / \mathrm{s}$ using ink 2 and same dispensing heights as the tip sizes 
Writing speed which portrays how fast the filament is deposited on the substrate is a key parameter that determines the features of the filament. Selection of appropriate writing speed that matches the tip size and the pressure is of importance in any printing operation. Printing with speed lower than required would lead to excessive deposition of material which causes over spreading of the feature. Bulges are also created at some locations along the written line and consequently lead to deposition of an irregular-shaped line. On the other hand, lines written using too high speed would thin out at intervals forming needlelike shapes. The lines, then, may become discontinuous or non-uniform in width due to the needlelike regions. An inversely proportionality relationship is found to exist between writing speed and feature spread. An increase in writing speed causes reduction in spread and, conversely, a decrease in speed results in an increase in feature spread.

The printing mechanism as affected by the writing speed involves a complex interaction with other material properties, such as, viscosity, surface energy and elasticity. When printing at speed higher than the exit speed of the ink from the nozzle, there is tendency for the filament to be stretched after its first contact with the substrate. This applied tensile stress on the filament increases as deposition speed increases, thus, decreasing the cross-sectional area and elongating its longitudinal dimension. How long the stretching persists before complete discontinuity in deposited feature depends on the elasticity of the ink. For lines written at 1, 3 and $5 \mathrm{~mm} / \mathrm{s}$ using different pressures, different tip sizes and ink with different viscosities shown in figures 4.20 to 4.23, the inverse proportionality relationship is maintained. 


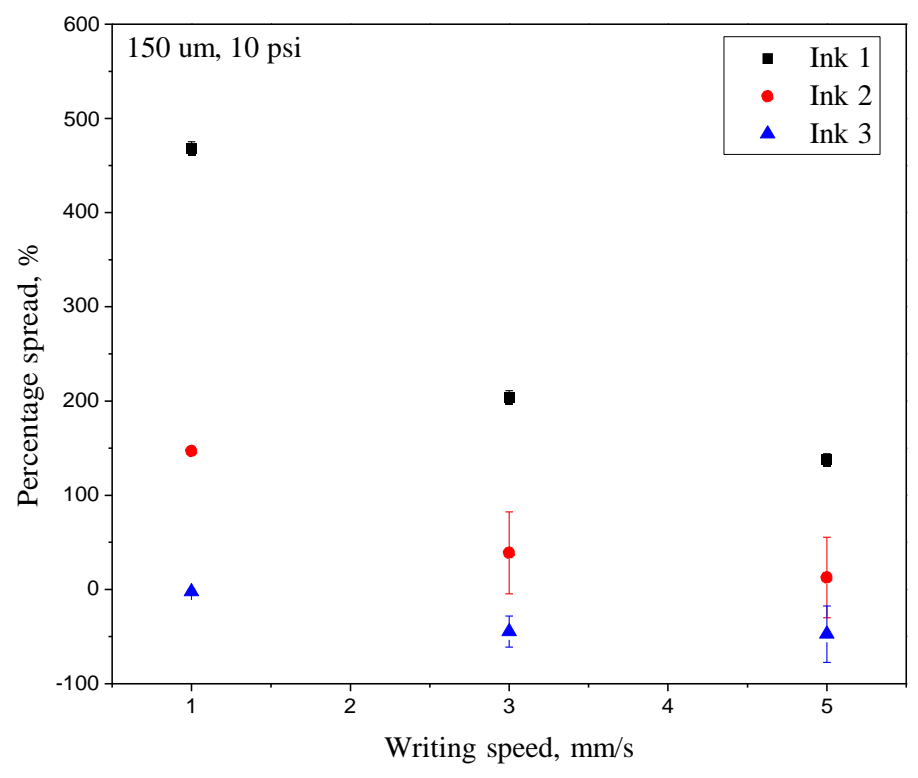

Figure 4.20 Spread versus writing speed for features printed with different inks at 10 psi using $150 \mu \mathrm{m}$ tip size and dispensing height

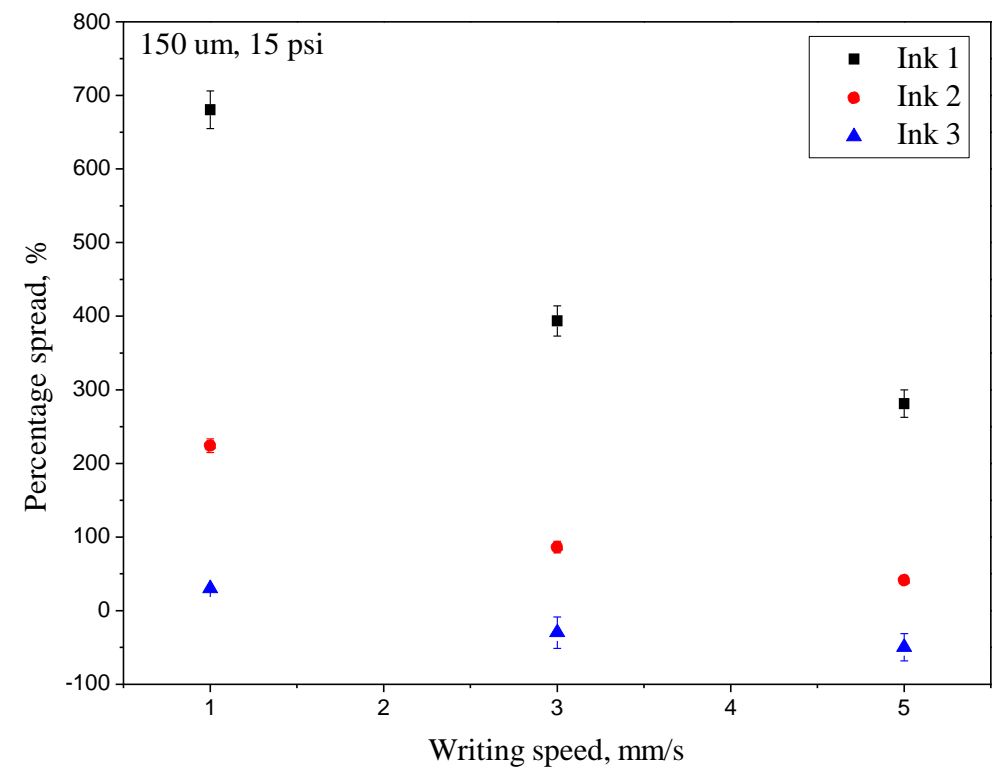

Figure 4.21 Spread versus writing speed for features printed with different inks at 15 psi using $150 \mu \mathrm{m}$ tip size and dispensing height 


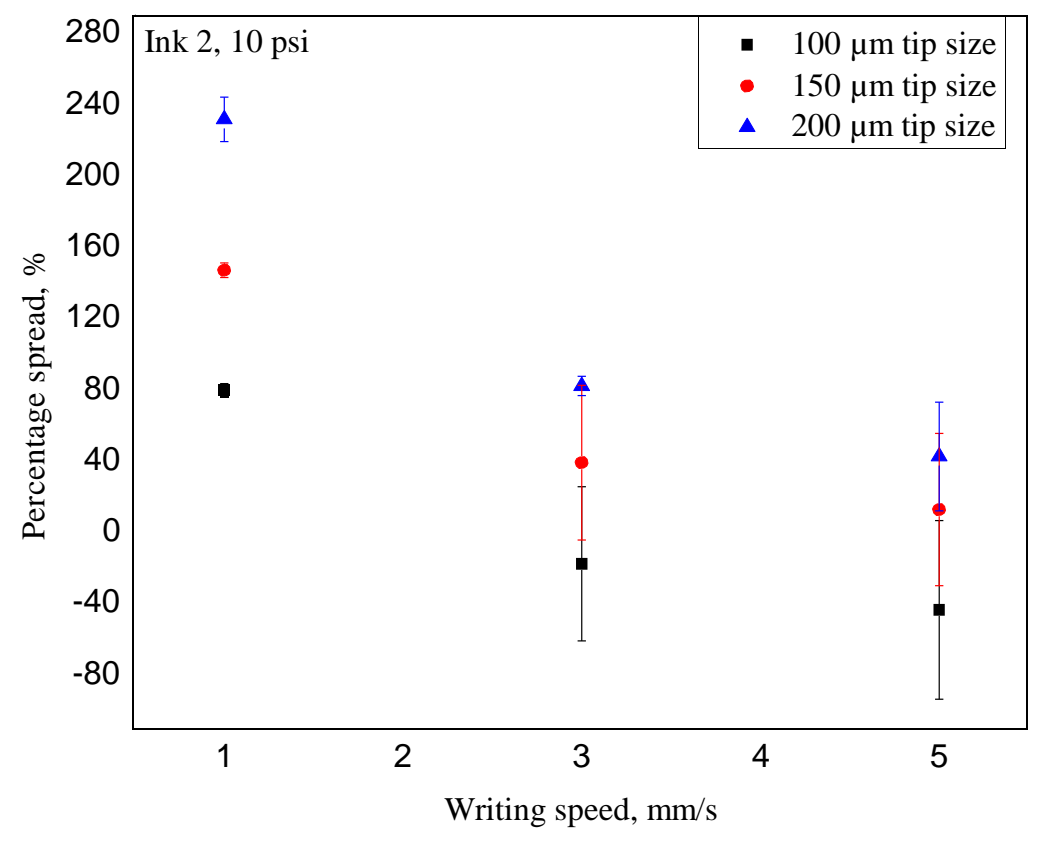

Figure 4.22 Spread versus writing speed for features printed with different tip sizes at 10 psi using ink 2 and same dispensing heights as the tip sizes

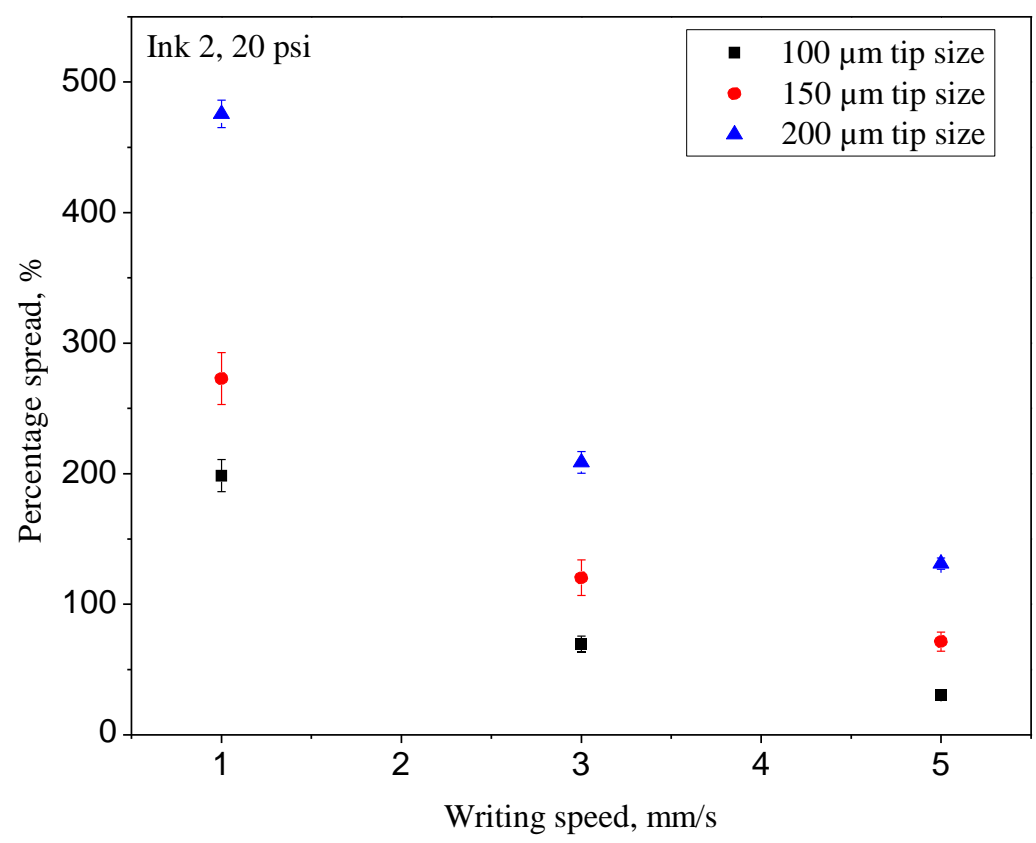

Figure 4.23 Spread versus writing speed for features printed with different tip sizes at 20 psi using ink 2 and same dispensing heights as the tip sizes 
It is also observed that deviation from linearity of the spread-writing speed relationship increases as the viscosity decreases. The deviation from linearity becomes more pronounced for ink 1 at higher pressure of $20 \mathrm{psi}$ as shown in figure 4.20. Higher standard deviation observed for ink 2 and ink 3 while printing with low pressure of 10 psi and small tip size of 100 and $150 \mu \mathrm{m}$, and high speed of 3 and $5 \mathrm{~mm} / \mathrm{s}$ results from printing of non-uniform patterns occasioned by inappropriate combination of printing parameters (Figure 4.22).

The feature profiles of the as deposited patterns obtained from profilometry measurements and shown in figures 4.24 to 4.27 show the shape of the deposited features as affected by the materials and process parameters. The widths and heights of the deposited features measured from the drawn profiles show decrease as writing speed increases. Figure 4.24 shows the profiles of features printed with ink 2 and 10 psi pressure using $100 \mu \mathrm{m}$ nozzle size for different writing speeds. The plot shows that the aspect ratio, which is the ratio of profile height to profile width, also increases as writing speed increases. The aspect ratio of 0.0326 at writing speed at $1 \mathrm{~mm} / \mathrm{s}$ increased to 0.0345 at $3 \mathrm{mms}$ and further increased to 0.0400 at speed of $5 \mathrm{~mm} / \mathrm{s}$. However, almost a reverse of this trend was obtained at $15 \mathrm{psi}$ as aspect ratios of $0.0341,0.0326$ and 0.0329 were obtained at printing speeds of 1,3 and $5 \mathrm{mms}$ respectively (Figure 4.25). These differences in aspect ratio trend are a result of the larger quantity of material deposited by the use of higher extrusion pressure. The effect of gravity becomes more pronounced at deposition of larger material quantity.

While printing with ink 3 , using $150 \mu \mathrm{m}$ nozzle, aspect ratio increased from 0.05 , to 0.06 and to 0.065 at 1,3 and $5 \mathrm{~mm} / \mathrm{s}$ for 10 psi printing pressure and 0.0590 .0620 .08 for pressure of 15 psi. Difference in viscosity also affected the aspect ratio as more viscous material has a prospect for shape retention when deposited. For the high viscosity ink 3, increase in pressure caused an 
increase in aspect ratio for all printing speeds (Figure 3.26 and 3.27). These changes observed in aspect ratio can be attributed to the effect of surface energy and gravity.

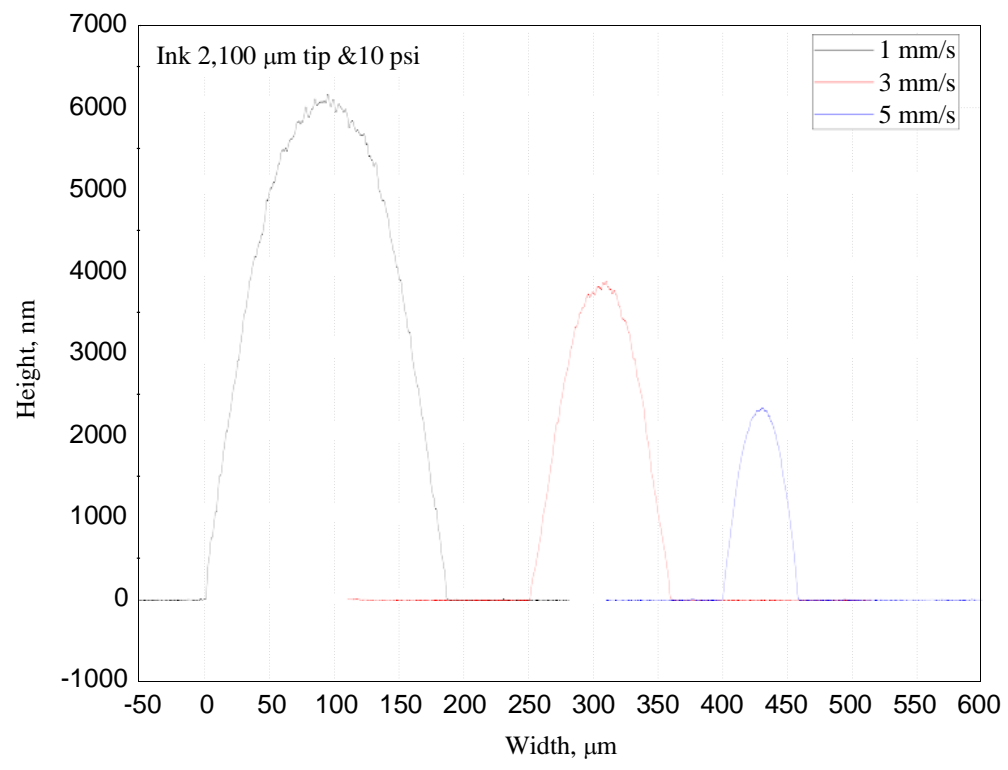

4.24 Profile of feature written with ink $2,100 \mu \mathrm{m}$ tip size and $10 \mathrm{psi}$ at different writing speeds

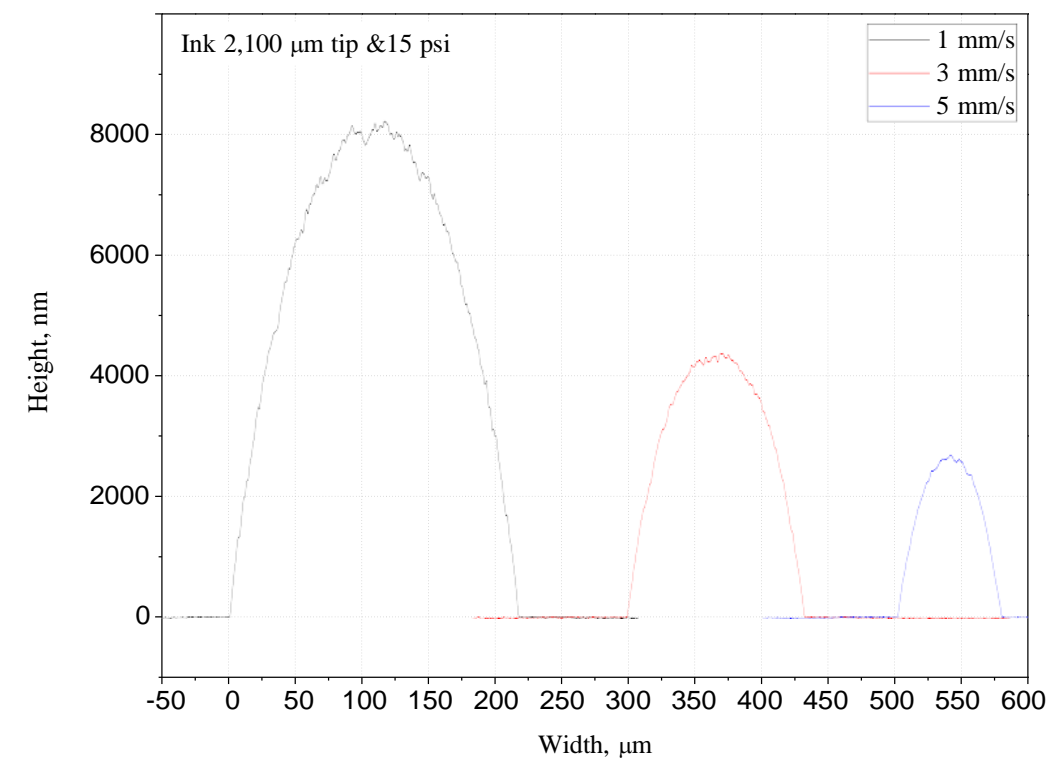

4.25 Profile of feature written with ink $2,100 \mu \mathrm{m}$ tip size and 15 psi at different writing speeds 


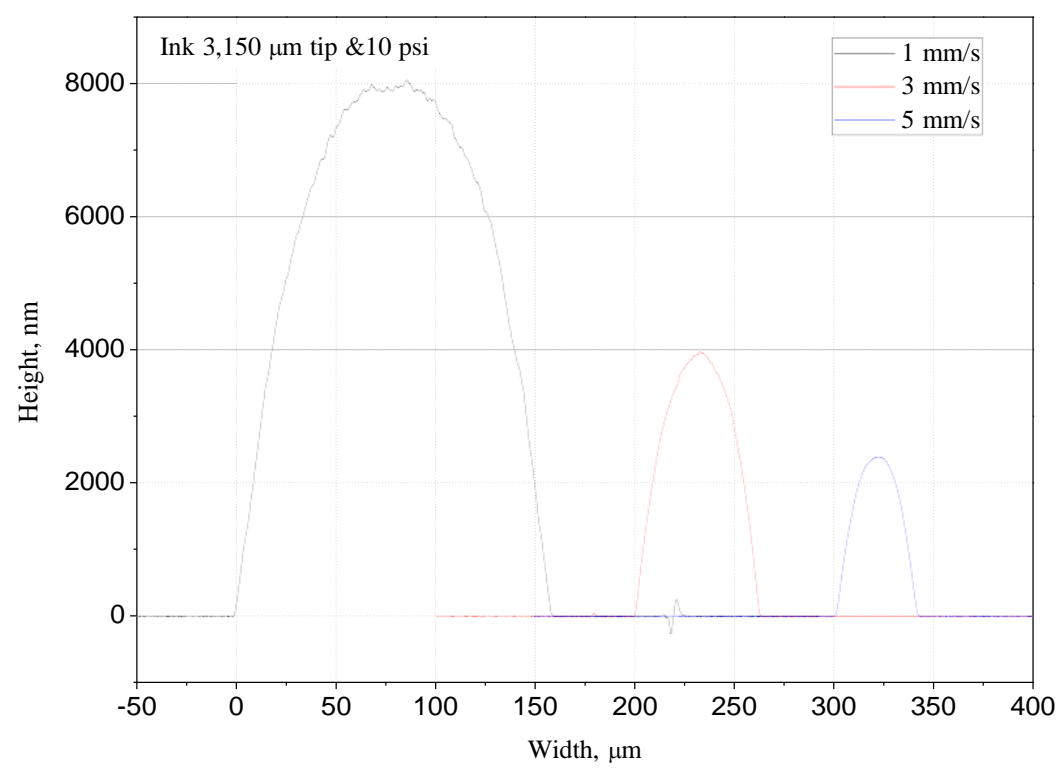

4.26 Profile of feature written with ink $3,150 \mu \mathrm{m}$ tip size and $10 \mathrm{psi}$ at different writing speeds

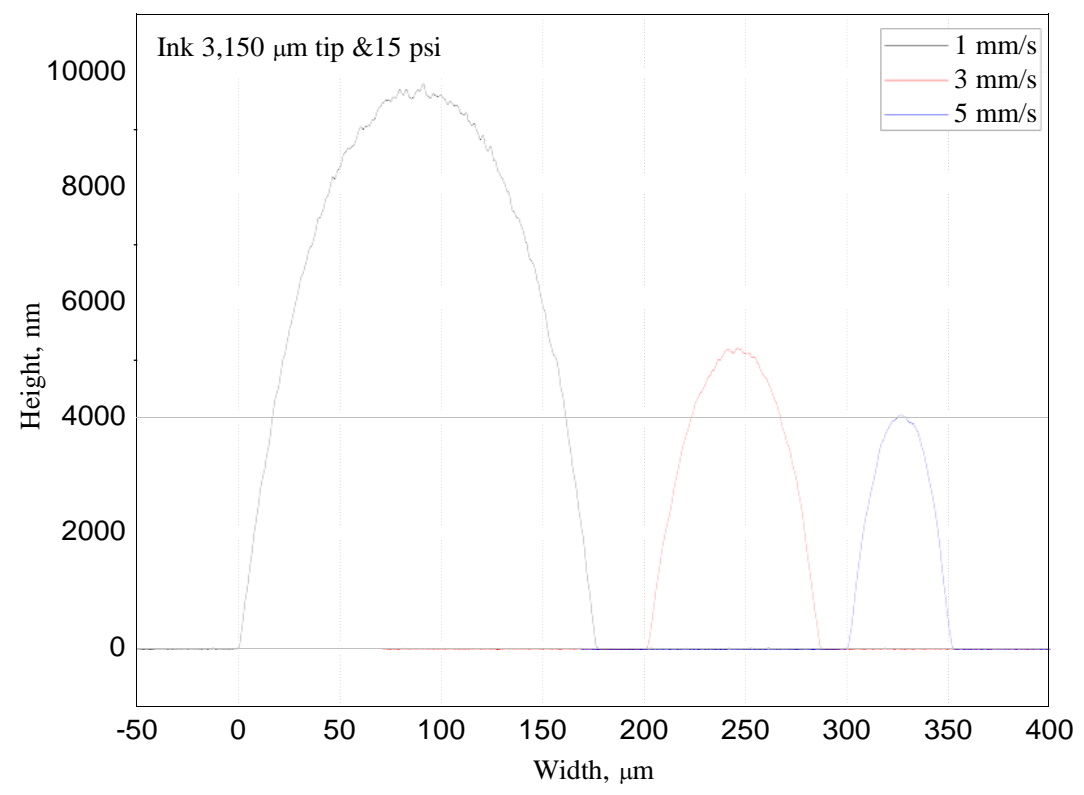

4.27 Profile of feature written with ink $2,100 \mu \mathrm{m}$ tip size and 15 psi at different writing speeds 


\subsection{Significance and Interactions of Printing Parameters}

The significance of the printing parameters on the width of the printed features and the interactions among them were investigated through a factorial analysis. Analysis of variance (ANOVA) was used to explore the influence of the printing parameters on the profile width and inter-connections between selected factors and levels. The effect of a factor is said to be significant if the F-value, computed as a ratio of the mean squared deviations to mean squared errors is greater than the critical value of $\mathrm{F}$, obtained based on the degree of freedoms. A larger F-value suggests that a particular factor has greater significance on feature width [76].

In this work, the ANOVA was performed in two sets. This becomes necessary in order to prevent excessive run of experiments and save both time and cost without sacrificing the integrity of the results obtained. Since there are four factors to be considered, and they are viscosity, tip size, extrusion pressure and writing speed. At first, viscosity, extrusion pressure and writing speed were taken as factors in the ANOVA with each having three levels. Based on the F-values presented in table 4.1, all the three factors have significant effects on the width of the printed feature. In comparison to other factors, viscosity has the largest F-value, thus, the most significant of the factors, and as such, plays the greatest role in determining the feature width. Speed and pressure also show significance, with writing speed being more important than pressure in controlling feature width.

Interactions among the factors are also seen to be significant as they have F-values greater than critical F-value at $95 \%$ confidence level. However, speed and viscosity shows the highest interaction, followed by viscosity and pressure interaction. The high speed and viscosity interaction is expected, as a high viscosity ink printed at high speed would produce a continuous pattern, while printing a low viscosity ink at high speed leads to deposition of discontinuous 
feature. Weak interaction, though, significant, exists between speed and pressure, and speed, viscosity and pressure.

Table 4.1 Analysis of variance (ANOVA) for feature width based on viscosity, writing speed and extrusion pressure

\begin{tabular}{lccccc}
\hline & Sum of & & & & F critical at 95\% \\
Source & square & DF & Mean square & F-value & confidence level \\
& & & & & \\
\hline Speed & 5711272 & 2 & 2855636.24 & 289.1003 & 3.428 \\
Viscosity & 22777753 & 2 & 11388876.5 & 1152.993 & 3.428 \\
Pressure & 2556321 & 2 & 1278160.29 & 129.399 & 3.428 \\
Speed x viscosity & 3036490 & 4 & 759122.544 & 76.85242 & 2.8661 \\
Speed x pressure & 297238.9 & 4 & 74309.7168 & 7.523003 & 2.8661 \\
Viscosity x pressure & 1928765 & 4 & 482191.371 & 48.81633 & 2.8661 \\
Speed x viscosity x & & & & & \\
pressure & & & & & \\
\hline
\end{tabular}

Considering tip size in place of viscosity in the previous analysis, giving the three factors in ANOVA to be tip size, writing speed and extrusion pressure. All the factors also play significant role in determining the feature width of the printed patterns, owing to their F-values shown in table 4.2. The tip size produced the highest F-value and is found to have the most significant effect on the feature width. This is followed by writing speed and pressure in that order. The interaction between speed and tip size is the most significant of all the interactions. While interactions among other factors are significant, they are however weak, as only slight differences exist between their F-values and corresponding critical F-values. This is believed to be an acceptable finding, because, if we consider an extreme case of low viscosity ink, in a nozzle with large diameter, little or no 
pressure would be required to extrude the ink from the nozzle and so, pressure would do little in controlling the width of the feature. However, in such a situation the speed at which the nozzle is moved across the surface would have profound impact on the nature of the deposited feature.

Table 4.2 Analysis of variance (ANOVA) for feature width based on tip size, writing speed and extrusion pressure

\begin{tabular}{lccccc}
\hline & Sum of & & & & F critical at 95\% \\
Source & square & DF & Mean square & F-value & confidence level \\
\hline Speed & 4322362.691 & 2 & 2161181.345 & 288.0499 & 3.428 \\
Tip size & 8353072.687 & 2 & 4176536.343 & 556.6635 & 3.428 \\
Pressure & 1384202.875 & 2 & 692101.4374 & 92.24573 & 3.428 \\
Speed*tip size & 1074690.421 & 4 & 268672.6052 & 35.80963 & 2.8661 \\
Speed*pressure & 214354.9992 & 4 & 53588.7498 & 7.142498 & 2.8661 \\
Tip size*pressure & 408782.6501 & 4 & 102195.6625 & 13.621 & 2.8661 \\
Speed*tip size* & & & & & \\
pressure & & & & & \\
\end{tabular}

Given the results obtained in the two sets of ANOVA above, viscosity can be said to be the most important factor in controlling the width of printed patterns, as it has profound effects both during extrusion of the filament from the nozzle, and even after deposition on the substrate. The next to viscosity in controlling feature width is the tip size used for printing. This is understandable, because, the resistance offered to the flow of a given fluid in a channel is inversely related to the channel size, and therefore, the quantity of material available for deposition is directly related to 
the tip size. The next in line is writing speed and followed by pressure. However, viscosity and tip size can be classified as static factors as they can easily be fixed based on the desired end feature. On the other hand, writing speed and extrusion pressure are dynamic in nature and can only be selected within a wide range of values to suit an already chosen viscosity and tip size. In respect of this, we might be tempted to say that, writing speed and pressure are the most important control parameters to optimize, in order to efficiently print patterns with uniform structure due to their dynamic nature.

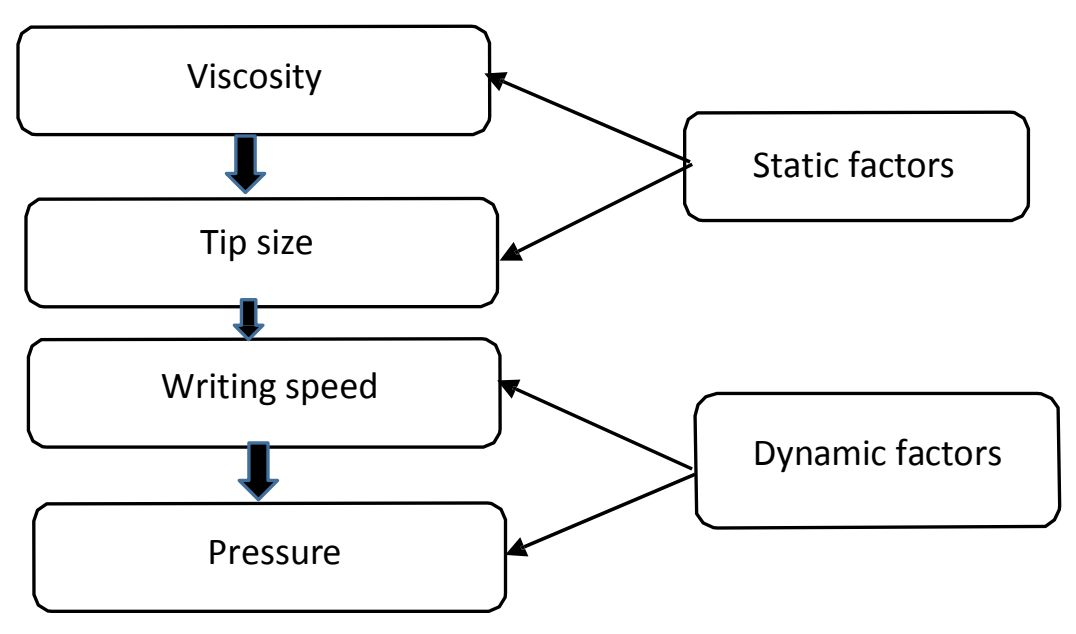

\section{Figure 4.28 Order of importance of printing parameters}

Having established the existence of interactions among all the printing parameters, it will therefore be difficult to completely isolate one for detailed discussions on how the properties of the deposited feature is affected by them. As a result, an estimate of the volumetric flow rate of material from the nozzle tip based on the pressure being applied, the inner diameter of the nozzle and the ink viscosity was estimated. The Poissule's equation (Equation 4.2) was used in estimating the volumetric flow rate, Q. [77]. In doing this, apart from the general assumptions for Poissule's 
equation, which are: (1) pressure is the predominant force in the system, (2) the flow is laminar and (3) the fluid is a Newtonian viscous fluid, additional assumption is taken in that the air pressure being applied in the syringe is equal to the pressure at the nozzle entrance.

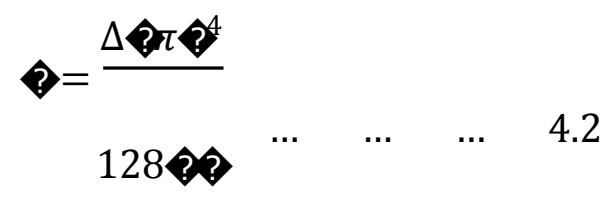

Where $\Delta \mathrm{P}$ is the pressure difference at both ends of the nozzle, and was taken as the air pressure used for the printing in $\mathrm{Pa}, \mathrm{d}$ is the inner diameter of the nozzle in $\mathrm{m}, \mathrm{u}$ is the dynamic viscosity of the ink used in $\mathrm{Pa} \mathrm{s}$, and $\mathrm{L}$ is the nozzle length in meters.

Figure 4.29 shows a three dimensional plot of feature width versus writing speed and volumetric flow rate of the ink from the nozzle. It is observed in the plot that as volumetric flow rate increases, feature width increases, but feature width decreases with an increase in writing speed.

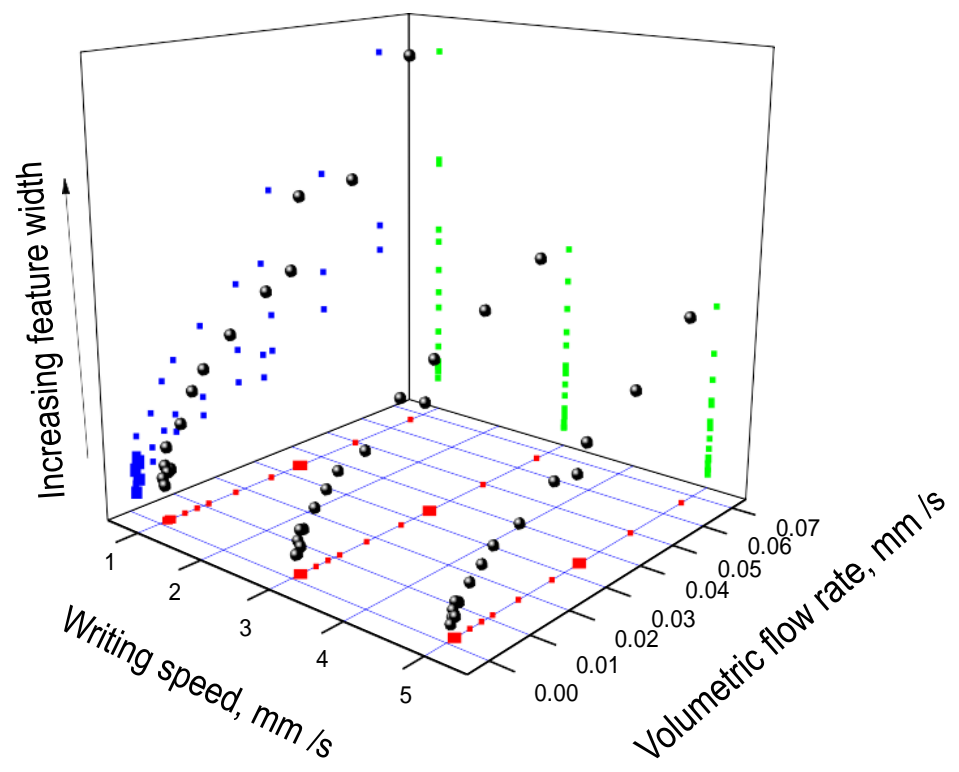

Figure 4.29 Feature width versus writing speed and volumetric flow rate 


\subsection{Printing Mechanism with the Extrusion Type Flow- Based DW}

The process, the mechanism and the optimization required while printing features through the use of NBRD can therefore be said to involve the following procedure. First step is the synthesis of ink with viscosity appropriate for the desired use. For instance, dots, planar arrays, gap spanning electrodes, scaffold printing in three dimensions, all requires inks with different properties. The next is the selection of tip size to be used for the printing, the inner diameter of the tip selected should be close enough to the width of the desired end printing. This becomes necessary, since, there is a minimum or maximum feature width that can be reached at a given tip size.

Optimization of the extrusion pressure to be used for printing is the next requirement. Extrusion pressure lower or higher than required would have adverse effect on the geometry of the printed feature. The dispensing height is thereafter optimized to match the pressure and the tip size, already set. Given an adequate pressure and dispensing height, the first set of filament extruded from the tip makes contact with the substrate and adheres to it. In order to draw a pattern of a given length, the nozzle needs to move across the substrate surface. The speed at which the nozzle traverses the surface, also requires optimization. Printing with too low speed results in features with excessive spread, and on the other hand, too high speed causes thinning and discontinuity in features. Schematic representation of the process and mechanism of printing is shown in figure 4.30 . 


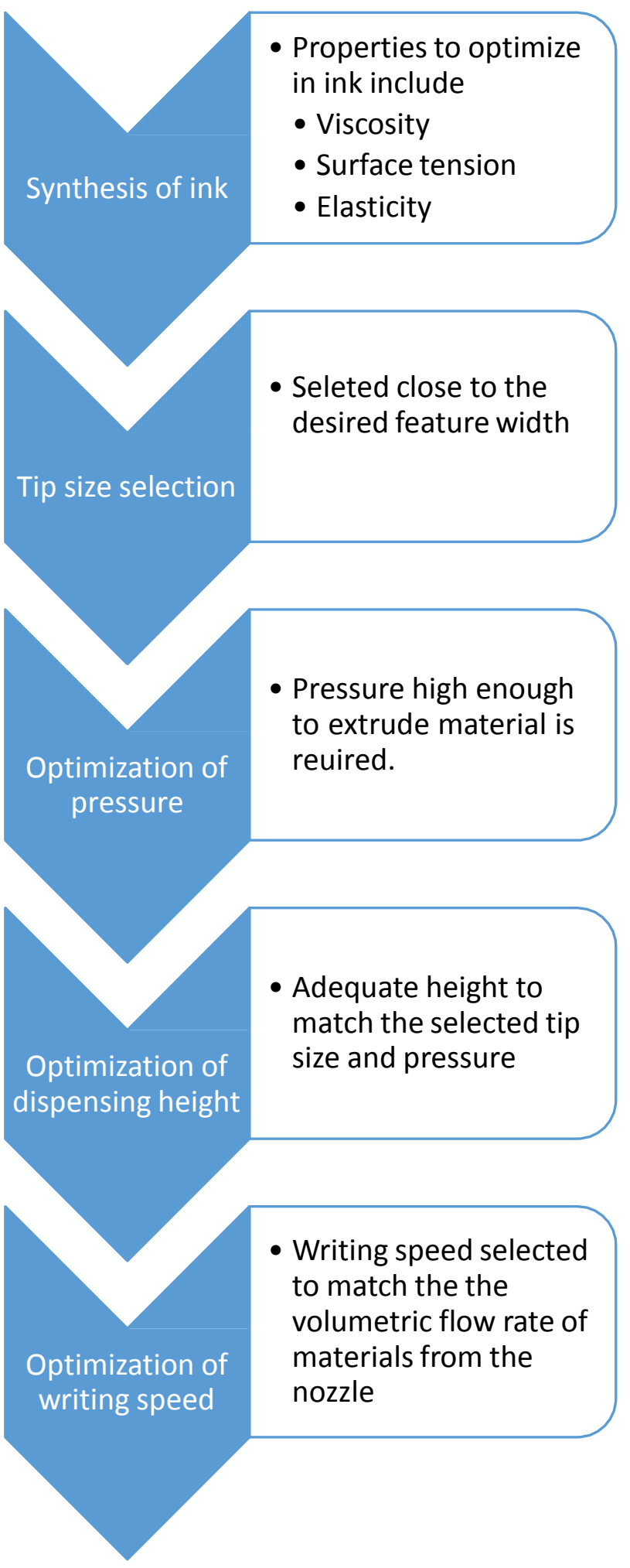

Figure 4.30 Schematic representation of the printing process and mechanism 


\subsection{Effect of Post Deposition Treatment on Feature Geometry}

Post deposition treatment is often required to make deposited films functional. However, a careful control of this is required, as it can produce a telling effect on the morphology and structural properties of the materials. Therefore, before heat treatment (sintering), a TGA of the inks were obtained and is shown in figure 4.31. The samples were heated in the TGA furnace to a high temperature of $800^{\circ} \mathrm{C}$ at the rate of $10^{\circ} \mathrm{C}$ per minute and was held at $800^{\circ} \mathrm{C}$ for 15 minutes. The results obtained from the TGA experiment is shown in figure 4.31 as a plot of weight versus temperature for the three inks. The three inks show similar burning behavior with sharp drop in weight at the early stage until drying and vaporization of solvent and stabilizer completes at around $200^{\circ} \mathrm{C}$. Weight reduction slowed down between 200 and $400^{\circ} \mathrm{C}$ which shows that most of the solvent vaporization had taken place at lower temperature. Decomposition of the polymeric content started at $400^{\circ} \mathrm{C}$ and continued up to $500^{\circ} \mathrm{C}$, after which crystallization of the $\mathrm{ZnO}$ continues without resulting in any further loss in weight.

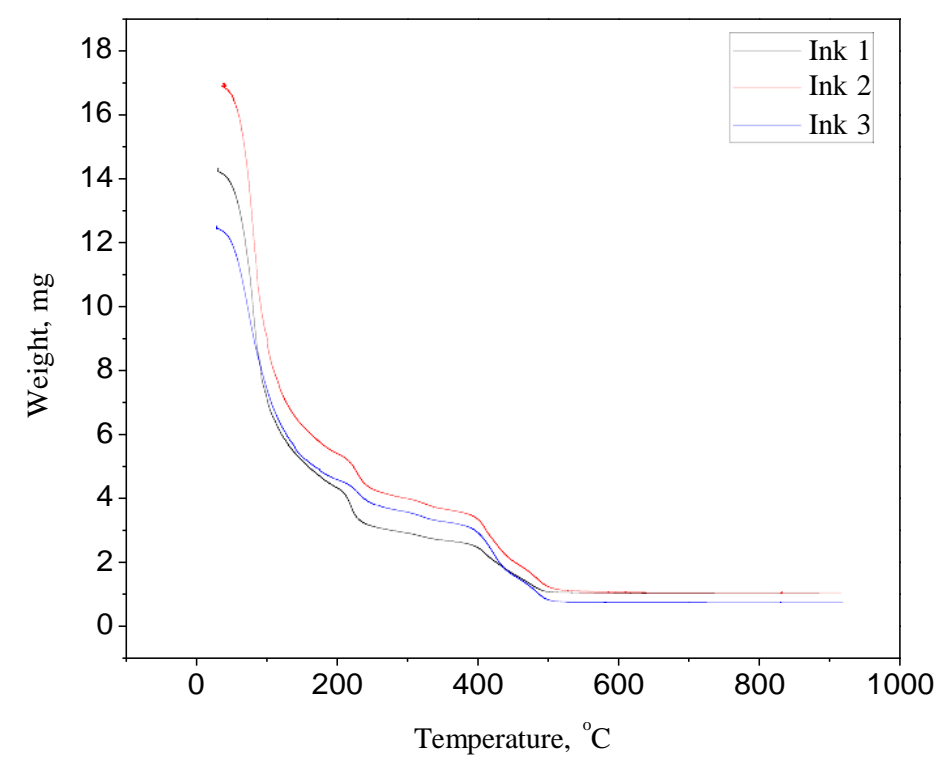

Figure 4.31 TGA of the inks used for printing 
Taking a clue from the burning behavior exhibited by the inks as reported above, after deposition, the films were first dried on hot plate at $200^{\circ} \mathrm{C}$ for ten minutes to dry and burn off the solvent, and was sintered in a convection furnace at $500^{\circ} \mathrm{C}$ for two hours to decompose the polymer and crystallize the $\mathrm{ZnO}$. The drying and sintering processes have both intrinsic and extrinsic effects on the deposited feature. The extrinsic effect is seen in the changes observed in the geometry of the features, in terms of the feature spread and profile, compared to before sintering geometry.

Figures 4.32 shows the before and after sintering spread of the feature printed with ink 2, $150 \mu \mathrm{m}$ tip size and 15 psi pressure, while, figure 4.33 depicts that of feature written with $200 \mu \mathrm{m}$ nozzle and 20 psi pressure. Both graphs show very slight reduction in spread after the feature was sintered at $500^{\circ} \mathrm{C}$ for two hours. This is an indication of the resistance offered to lateral reduction in size by the features during sintering, and the force of adhesion existing between the substrate and the film might be responsible for this behavior. Similar trend is observed for inks of different viscosities as shown in figures 4.34 and 4.35 for ink 1 and ink 2 respectively.

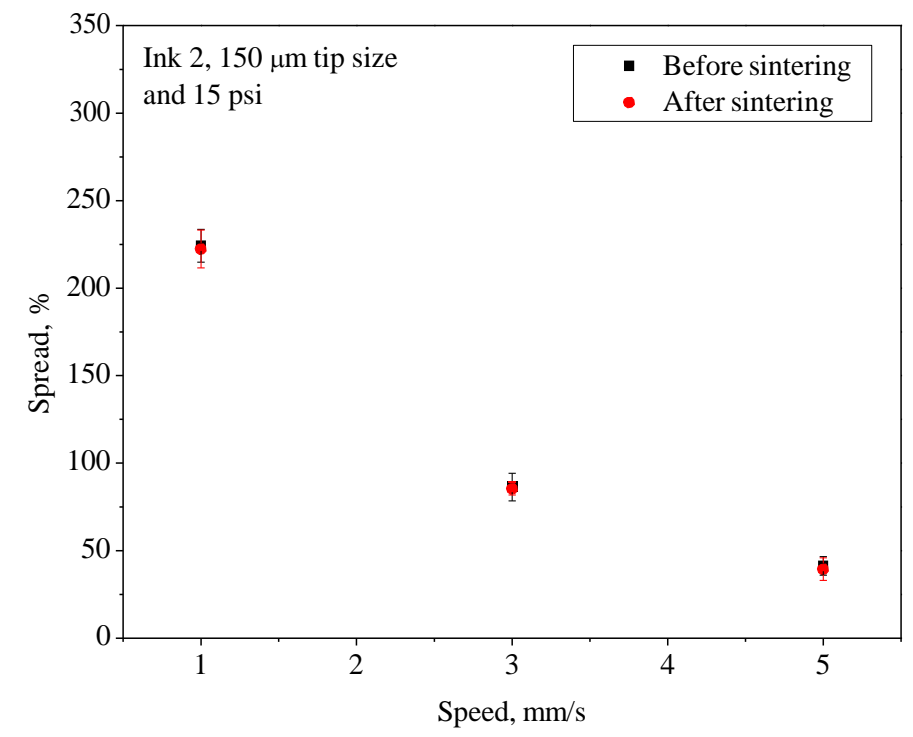

Figure 4.32 Before and after sintering spreads versus writing speed for feature printed with ink 2, $150 \mu \mathrm{m}$ nozzle size and 15 psi pressure 


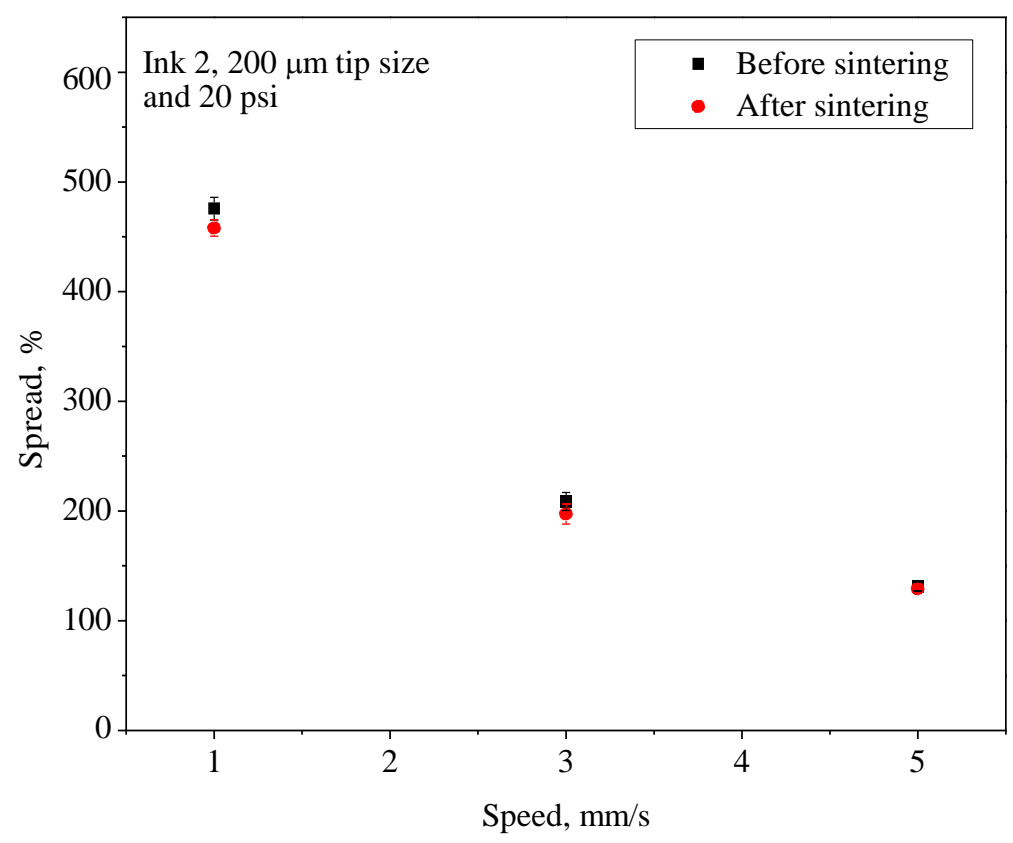

Figure 4.33 Before and after sintering spreads versus writing speed for feature printed with ink 2, $200 \mu \mathrm{m}$ nozzle size and 20 psi pressure

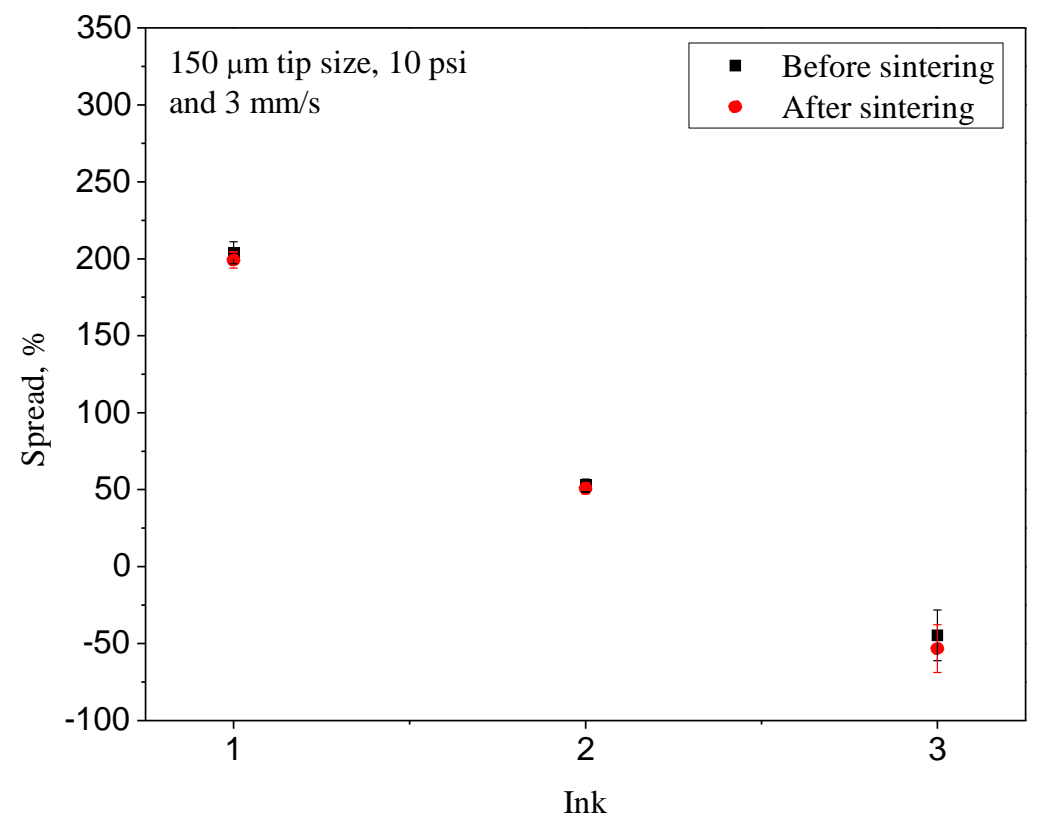

Figure 4.34 Before and after sintering spreads for inks with different viscosities printed with ink $150 \mu \mathrm{m}$ nozzle size, $10 \mathrm{psi}$ and $3 \mathrm{~mm} / \mathrm{s}$ speed 


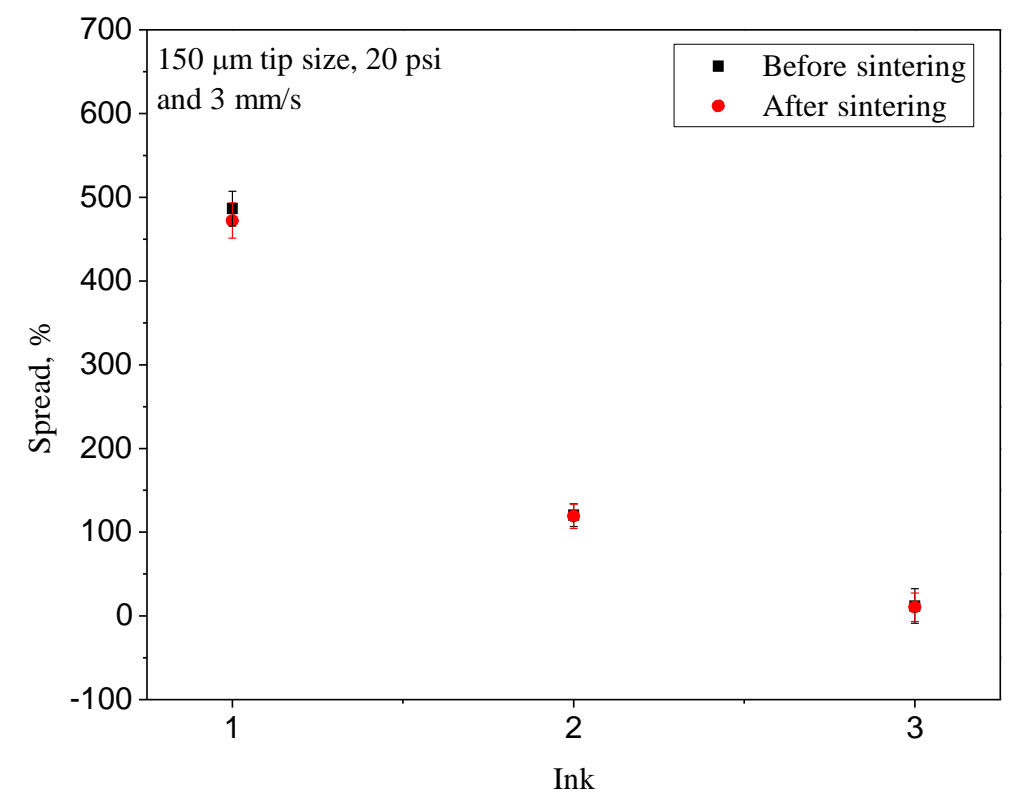

Figure 4.35 Before and after sintering spreads for inks with different viscosities printed with ink $150 \mu \mathrm{m}$ nozzle size, $20 \mathrm{psi}$ and $3 \mathrm{~mm} / \mathrm{s}$ speed

Profiles of as deposited features in comparison with that of sintered features for inks 2 and 3, printed with $150 \mu \mathrm{m}$ nozzle and 10 and 15 psi pressure at different writing speeds are shown in figures 4.36 and 4.37. While the widths of the sintered features still fall within the range observed in the earlier measure spread, a large reduction in height is observed. For instance, the height of feature printed with ink 2 using $150 \mu \mathrm{m}$ tip size and 10 psi at $1 \mathrm{~mm} / \mathrm{s}$ writing speed decreased from $6 \mu \mathrm{m}$ to about $800 \mathrm{~nm}$, signifying an $86 \%$ reduction in height, and bringing the aspect ratio from approximately 0.0315 to 0.00002 . Also, a height reduction of about $86 \%$ is observed for pattern printed with ink 3 using $150 \mu \mathrm{m}$ nozzle and 15 psi at $3 \mathrm{~mm} / \mathrm{s}$. 


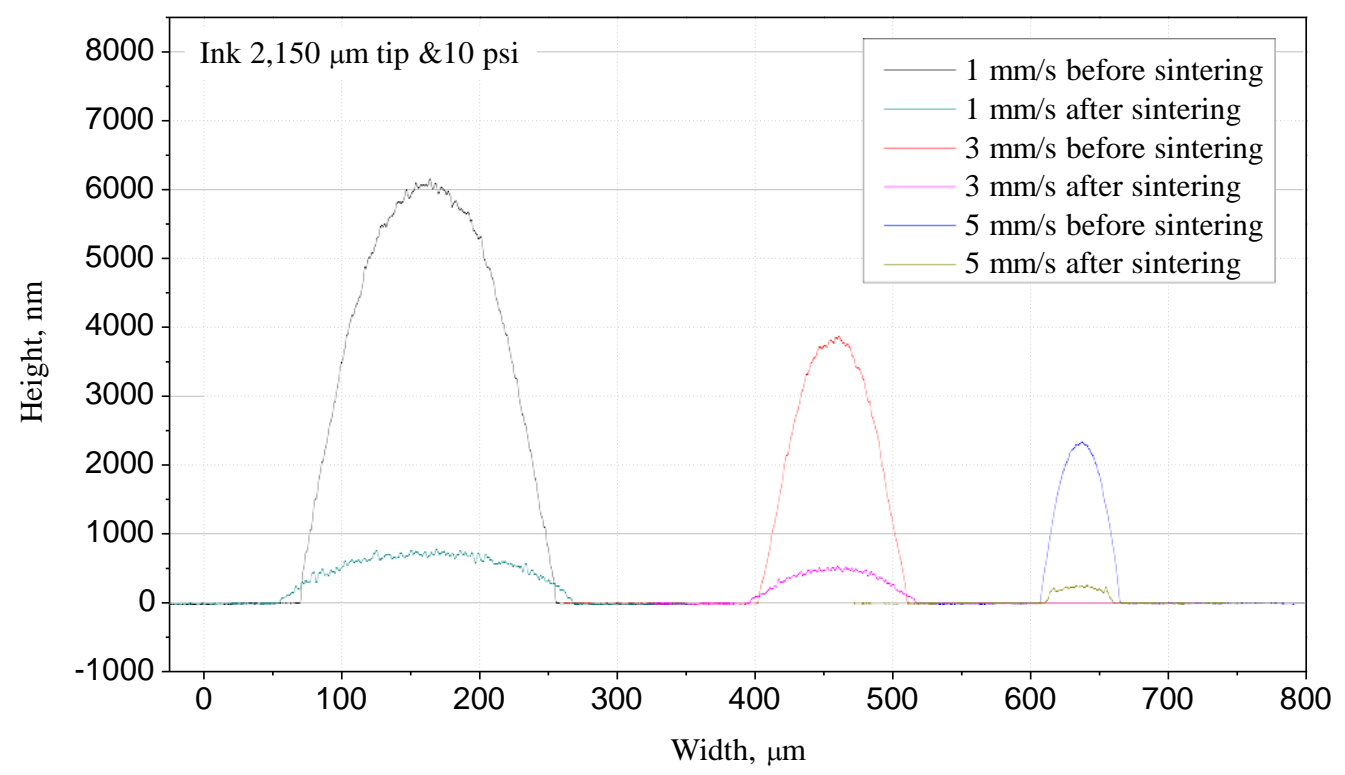

Figure 4.36 Before and after sintering profile for feature printed with ink 2, $150 \mu \mathrm{m}$ nozzle size, and 10 psi at different writing speed

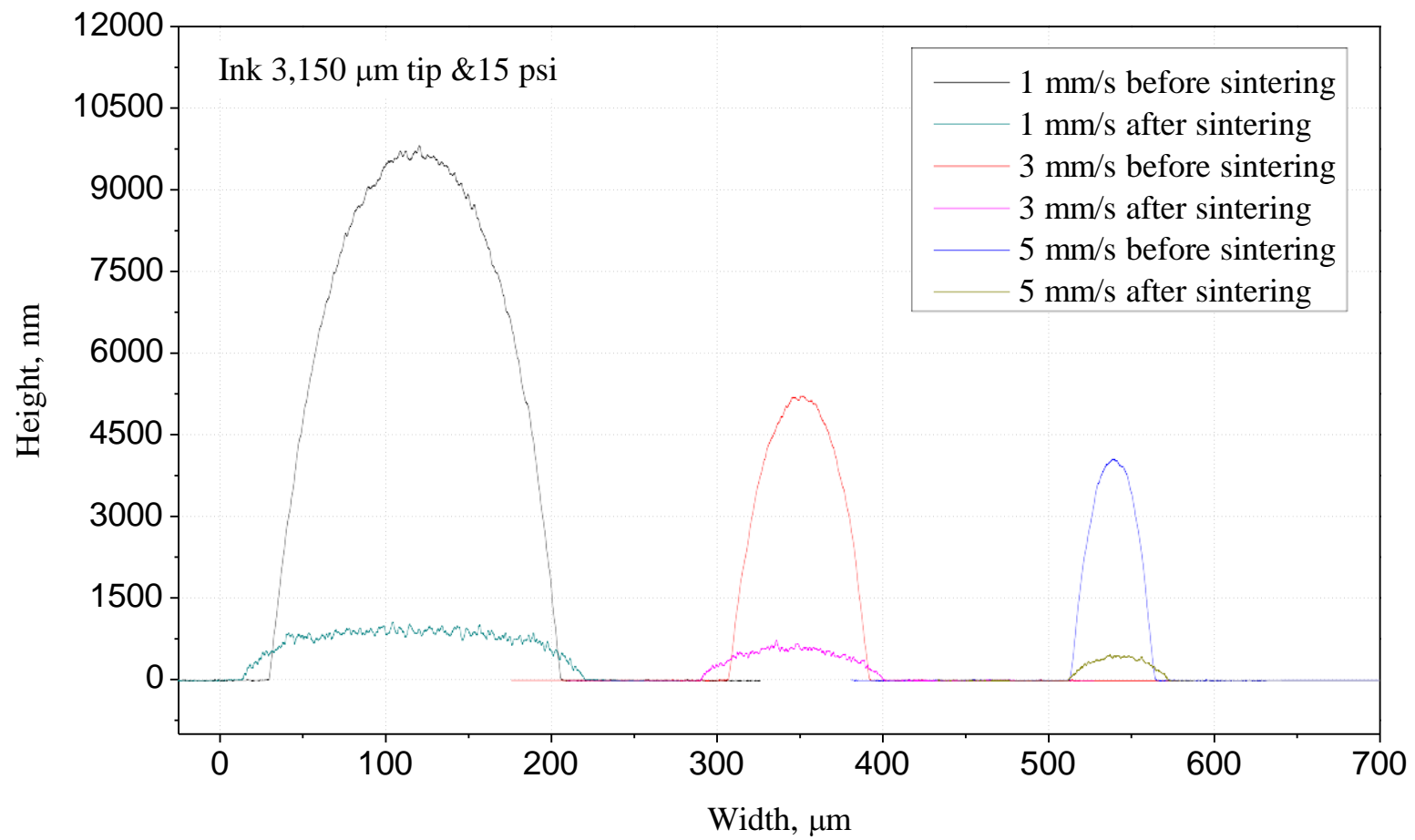

Figure 4.37 Before and after sintering profile for feature printed with ink 2, $150 \mu \mathrm{m}$ nozzle size, and $10 \mathrm{psi}$ at different writing speeds 


\section{EFFECT OF PRINTING ON RESULTING MICROSTRUCTURE OF WRITTEN PATTERN}

\subsection{Crystal Structure and Morphology}

Crystal orientation as a property of the deposited films is of critical importance in determining their areas of suitable applications. The XRD spectra of AZO thin films, printed on glass substrates with various printing parameters are shown in figure 5.1. The crystallographic data obtained from a Panalytical X-ray diffractometer and analyzed by using Pro high score software by comparing the obtained peaks with $\mathrm{ZnO}$ data from JCPDS, 36-1451. The patterns of the analyzed AZO data correspond to the reflection of hexagonal wurtzite-structured $\mathrm{ZnO}$ planes depicting both nanocrystalline and polycrystalline behavior (Figure 5.1). All the films were observed to show a strong (002) peak, indicating preferential orientation along the c-axis perpendicular to the substrate surface. The 002 direction of $\mathrm{ZnO}$ thin film is the most thermodynamically favorable growth direction and since it possesses minimum surface free energy along this plane, higher condensation rate of $\mathrm{Zn}^{2+}$ ions along this axis is favored [78]. Apart from the 002 peak, other $\mathrm{ZnO}$ peaks were also present, however the prevalent ones are the 100 and 101 peaks. Presence of the strong and sharp diffraction peaks indicates the films are well crystallized as a result of the sintering process. The intensities of these peaks vary from sample to sample based on the process parameters used. Detailed discussion of the influence of the printing parameters on the microstructure is presented later on in this work.

The XRD parameters along the 002 peak of the deposited AZO film, showing the lattice parameters as affected by the printing conditions is presented in table 5.1. The lattice constants, a and c, of the deposited films, calculated from the XRD data along the 002 peak ranges from 0.2891 
$\mathrm{nm}$ to 0.2924 for a and 0.5007 to $0.5064 \mathrm{~nm}$ for $\mathrm{c}$. The average grain size of the films was found to have minimum value of $11.67 \mathrm{~nm}$ and maximum value of $29.19 \mathrm{~nm}$. The values compares with the values for standard $\mathrm{ZnO}$ of 0.329 for a, and 0.5206 for $\mathrm{c}$, and those reported in the literature [51] [52]. The FWHM and the diffraction angles of the deposited films were found to be in the range of 0.2598 to $0.6494 \mathrm{~nm}$ and 34.5909 to $34.99962 \theta^{\circ}$. The disparity of the obtained values and the standard value $34.422 \theta^{\circ}$ for $\mathrm{ZnO}$ can be attributed to the $\mathrm{Al}$ doping and variations in film processing parameters. The diffraction angle value is however comparable to the value of 36.68 $2 \theta^{\circ}$ obtained by Foo et al. [39] for $\mathrm{ZnO}$ prepared using 2-methoxy ethanol as solvent. Inter lattice spacing with values ranging from 0.2564 to 0.2593 were obtained. Residual strain along c-axis, calculated using equation 5.1 [79], ranges from -0.0360 to -0.0269 . Negative values of strain obtained for all the films along the c-axis signify good crystal formation, as the films are not in tension along this direction.

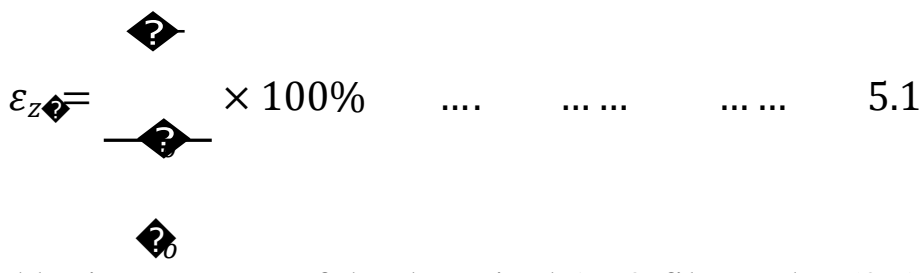

Where $\mathrm{c}$ is the strained lattice constant of the deposited AZO film and $\mathrm{c}_{\mathrm{o}}(0.5206)$ is the unstrained lattice constant for $\mathrm{ZnO}$.

The morphologies of films deposited on glass substrate using different printing parameters were examined under SEM after sintering for 2 hours at $500^{\circ} \mathrm{C}$. The SEM micrographs shown in figures 5.2 to 5.6 indicate that the films possess elongated worm-like structures which are dense and of uniform formation. The length of the worm-like structure decreases as the quantity of materials deposited increases. This behavior is noticeable in the SEM micrographs of figures 5.2 to 5.5. The structures becomes denser and more compact when the film was printed with a large nozzle 
compared to those obtained with smaller tip size nozzle. 
Table 5.1 XRD parameters along 002 peak for AZO films printed with different printing parameters

\begin{tabular}{|c|c|c|c|c|c|c|c|c|c|}
\hline & $\mathrm{P}$ & WS & $\begin{array}{l}\text { Peak } \\
\text { position } \\
\left(2 \theta^{\circ}\right)\end{array}$ & $\begin{array}{l}\text { FWHM } \\
\left(2 \theta^{\circ}\right)\end{array}$ & $\begin{array}{l}\mathrm{d}- \\
\text { spacing, } \\
(\mathrm{nm})\end{array}$ & $\begin{array}{l}\text { Lattice } \\
\text { constant, } \\
\text { a (nm) }\end{array}$ & $\begin{array}{l}\text { Lattice } \\
\text { constant, } \\
\text { c (nm) }\end{array}$ & $\begin{array}{l}\text { Grain } \\
\text { size, D } \\
(\mathrm{nm})\end{array}$ & $\begin{array}{l}\text { Strain, } \\
\varepsilon_{Z}(\%)\end{array}$ \\
\hline \multirow{4}{*}{$\begin{array}{l}\text { Ink } 1, \\
150 \\
\mu \mathrm{m} \\
\text { nozzle }\end{array}$} & 10 & 3 & 34.5909 & 0.3247 & 0.259314 & 0.292385 & 0.506426 & 23.35665 & -2.72261 \\
\hline & 15 & 1 & 34.7452 & 0.3247 & 0.258197 & 0.291127 & 0.504246 & 23.34683 & -3.14138 \\
\hline & 15 & 3 & 34.8932 & 0.4546 & 0.257136 & 0.28993 & 0.502173 & 16.66882 & -3.53951 \\
\hline & 15 & 5 & 34.7256 & 0.3897 & 0.258338 & 0.291286 & 0.504522 & 19.45374 & -3.08839 \\
\hline \multirow{5}{*}{$\begin{array}{l}\text { Ink } 2 \\
, 100 \\
\mu \mathrm{m} \\
\text { nozzle }\end{array}$} & 10 & 3 & 34.6129 & 0.3247 & 0.259154 & 0.292205 & 0.506114 & 23.35525 & -3.17243 \\
\hline & 15 & 1 & 34.8725 & 0.3897 & 0.257284 & 0.290097 & 0.502462 & 19.44593 & -3.15785 \\
\hline & 15 & 3 & 34.6597 & 0.2598 & 0.258815 & 0.291823 & 0.505452 & 29.18585 & -2.90111 \\
\hline & 20 & 3 & 34.7143 & 0.3247 & 0.25842 & 0.291378 & 0.504681 & 23.3488 & -3.6222 \\
\hline & 20 & 5 & 34.6266 & 0.3247 & 0.259054 & 0.292093 & 0.50592 & 23.35438 & -2.99037 \\
\hline \multirow{5}{*}{$\begin{array}{l}\text { Ink 2, } \\
150 \\
\mu \mathrm{m} \\
\text { nozzle }\end{array}$} & 10 & 3 & 34.7567 & 0.3247 & 0.258115 & 0.291033 & 0.504084 & 23.3461 & -2.78255 \\
\hline & 15 & 1 & 34.7513 & 0.3247 & 0.258153 & 0.291077 & 0.50416 & 23.34644 & -3.48403 \\
\hline & 15 & 3 & 34.9241 & 0.3897 & 0.256915 & 0.289681 & 0.501743 & 19.44317 & -2.9098 \\
\hline & 15 & 5 & 34.6565 & 0.3897 & 0.258838 & 0.291849 & 0.505497 & 19.4574 & -3.05782 \\
\hline & 20 & 3 & 34.6894 & 0.3247 & 0.2586 & 0.29158 & 0.505032 & 23.35039 & -2.81983 \\
\hline \multirow{5}{*}{$\begin{array}{l}\text { Ink 2, } \\
200 \\
\mu \mathrm{m} \\
\text { nozzle }\end{array}$} & 10 & 3 & 34.5913 & 0.3897 & 0.259311 & 0.292382 & 0.50642 & 19.46085 & -2.7237 \\
\hline & 15 & 1 & 34.7187 & 0.2598 & 0.258388 & 0.291342 & 0.504619 & 29.18116 & -3.06972 \\
\hline & 15 & 3 & 34.7954 & 0.2598 & 0.257836 & 0.29072 & 0.503541 & 29.17504 & -3.2768 \\
\hline & 15 & 5 & 34.6322 & 0.4546 & 0.259014 & 0.292047 & 0.505841 & 16.68071 & -2.83507 \\
\hline & 20 & 3 & 34.7822 & 0.3247 & 0.257931 & 0.290826 & 0.503726 & 23.34447 & -3.24123 \\
\hline \multirow{5}{*}{$\begin{array}{l}\text { Ink } 3 \\
150 \\
\mu \mathrm{m} \\
\text { nozzle }\end{array}$} & 10 & 3 & 34.7227 & 0.3897 & 0.258359 & 0.291309 & 0.504563 & 19.45389 & -3.08055 \\
\hline & 15 & 1 & 34.9179 & 0.3897 & 0.25696 & 0.289731 & 0.501829 & 19.4435 & -3.60562 \\
\hline & 15 & 3 & 34.9028 & 0.3897 & 0.257067 & 0.289853 & 0.50204 & 19.44431 & -3.56521 \\
\hline & 15 & 5 & 34.9996 & 0.6494 & 0.256379 & 0.289076 & 0.500694 & 11.66528 & -3.82362 \\
\hline & 20 & 3 & 34.8216 & 0.4546 & 0.257648 & 0.290508 & 0.503174 & 16.67209 & -3.34733 \\
\hline
\end{tabular}

$\mathrm{P}$ is the extrusion pressure and WS is the writing speed 


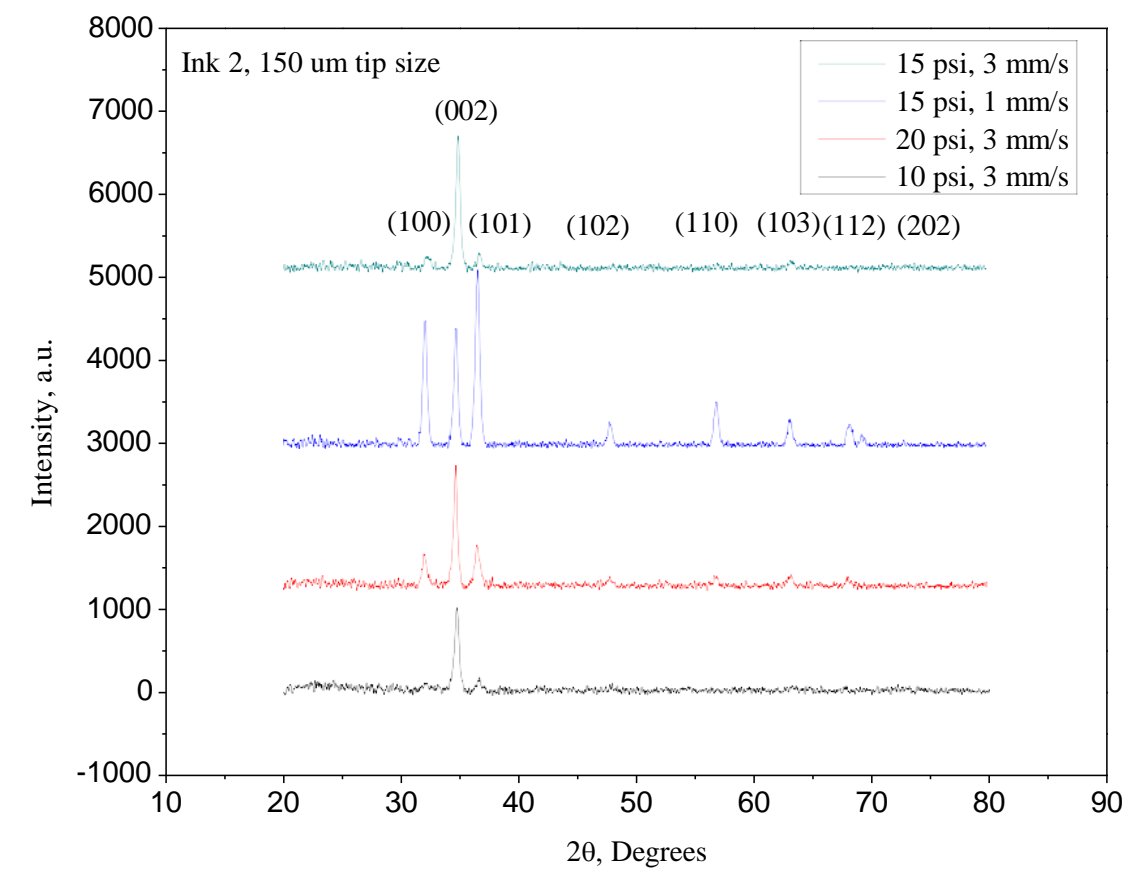

Figure 5.1 XRD spectra for AZO films deposited with different printing parameters

This is understandable, since, deposition of higher quantity materials means more materials are available for crystallization. Similar wormlike structures were obtained by spin coating of $\mathrm{ZnO}$ and were reported by Dhingra et al. [80] who used them for liquefied petroleum gas sensing. The nano-fiber structure and nano wall network obtained by Islam and Podder [81] and Su et al. [82] through spray pyrolysis and MBE respectively, also show similar pattern. The somewhat, rough surface, which can be as a result of precursor to stabilizer ratio or due to high temperature sintering, possessed by this structural formation might aid the functionality of the film for gas sensing purposes as larger surface area is exposed. Also, the columnar structure signifies connection among the grains as less grain boundaries will be encountered. 


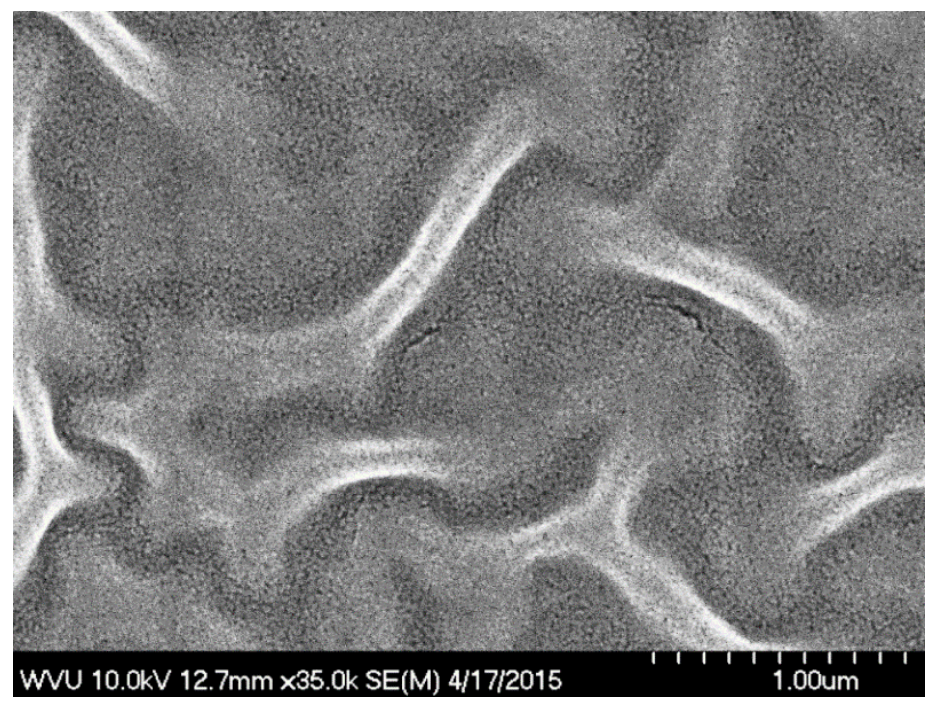

Figure 5.2 SEM images of feature printed with ink $2,100 \mu \mathrm{m}$ nozzle, $15 \mathrm{psi}$ and $3 \mathrm{~mm} / \mathrm{s}$, and sintered at $500^{\circ} \mathrm{C}$ for 2 hours

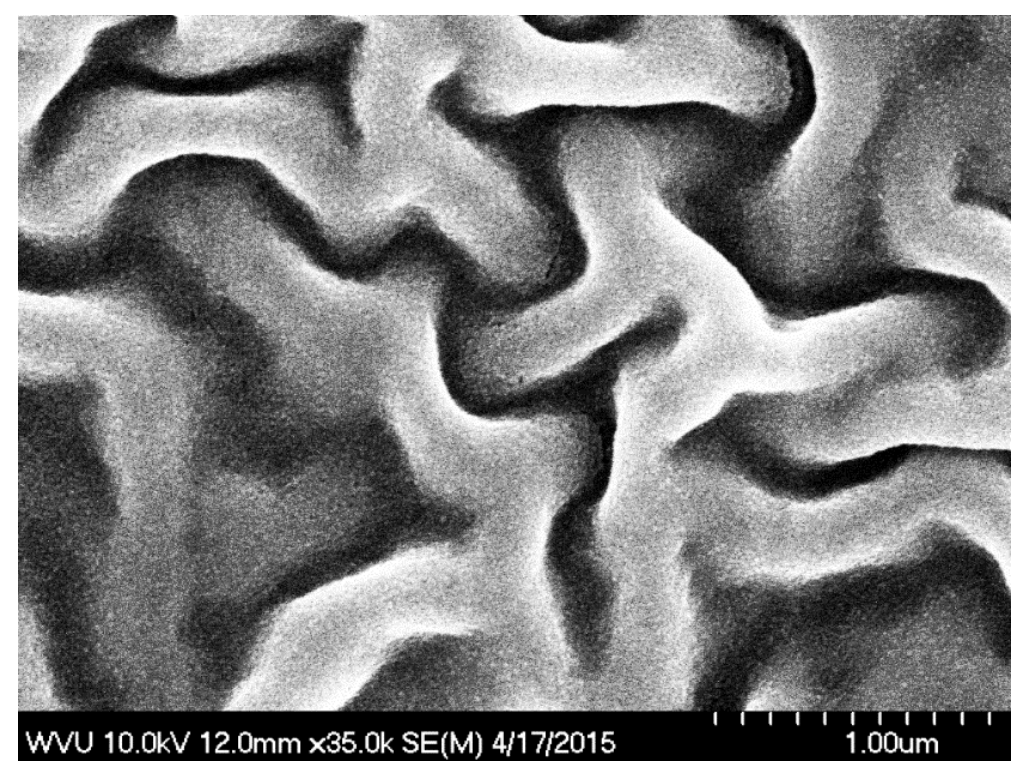

Figure 5.3 SEM images of feature printed with ink $2,150 \mu \mathrm{m}$ nozzle, $15 \mathrm{psi}$ and $3 \mathrm{~mm} / \mathrm{s}$, and sintered at $500^{\circ} \mathrm{C}$ for 2 hours 


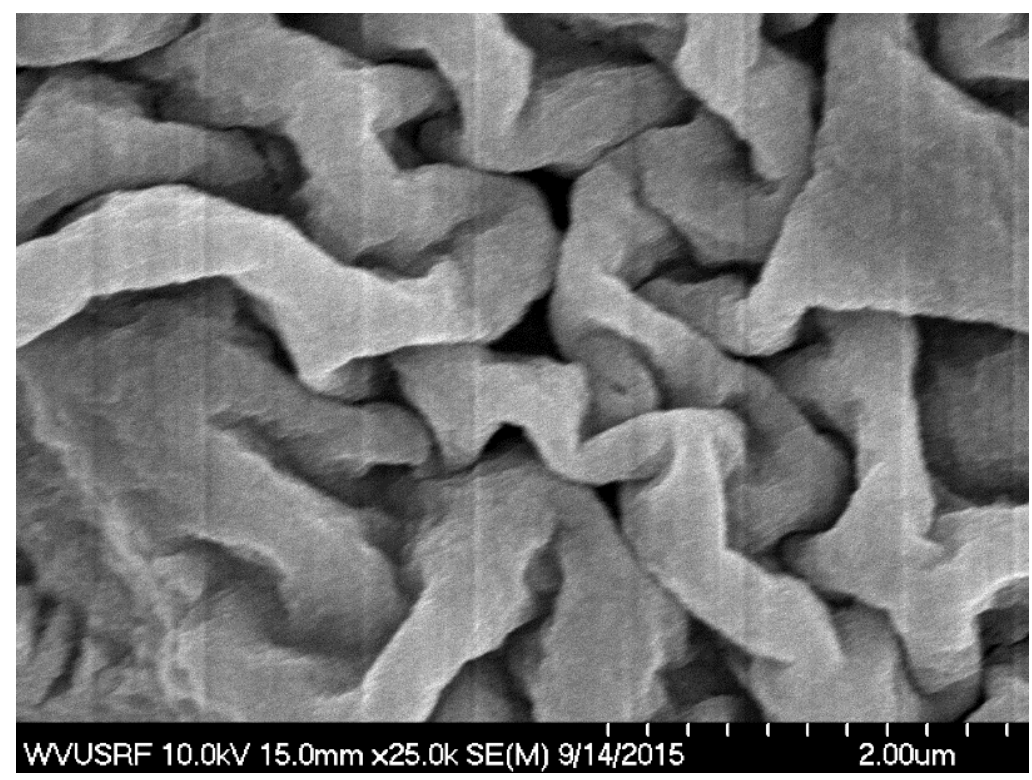

Figure 5.4 SEM images of feature printed with ink 2, $150 \mu \mathrm{m}$ nozzle, $15 \mathrm{psi}$ and $1 \mathrm{~mm} / \mathrm{s}$, and sintered at $500^{\circ} \mathrm{C}$ for 2 hours

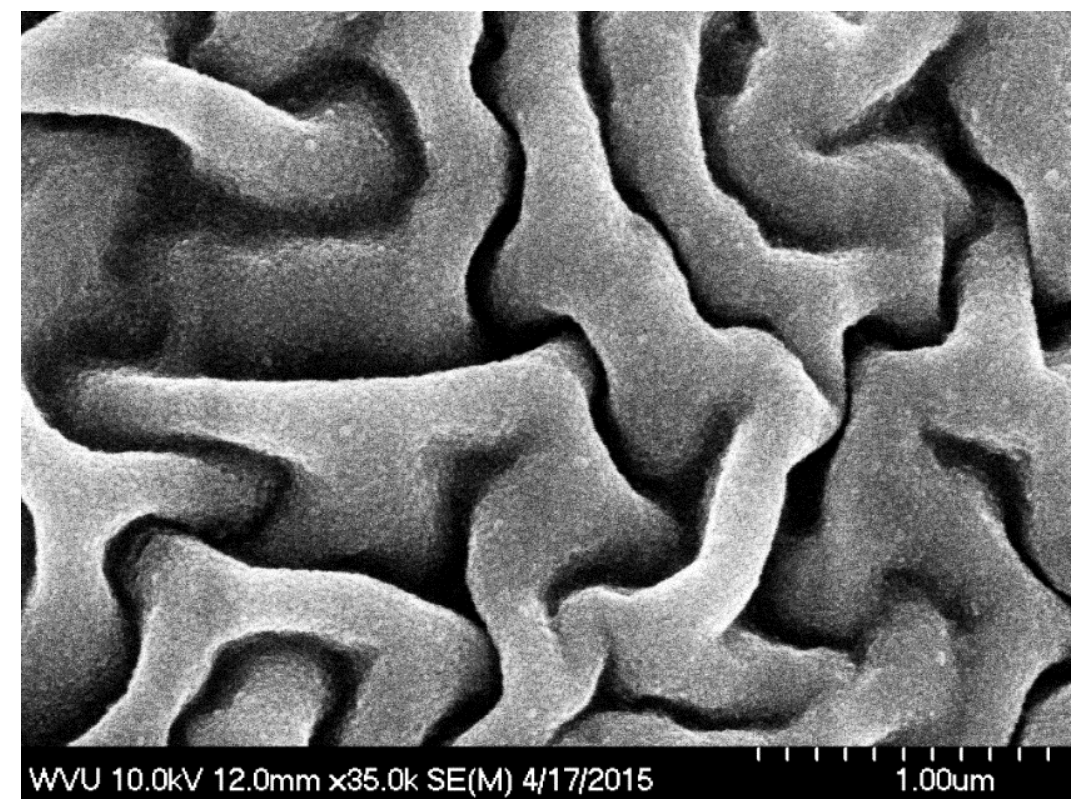

Figure 5.5 SEM images of feature printed with ink 2, $200 \mu \mathrm{m}$ nozzle, $15 \mathrm{psi}$ and $3 \mathrm{~mm} / \mathrm{s}$, and sintered at $500^{\circ} \mathrm{C}$ for 2 hours 


\subsection{Dependence of Micro Structure on Printing Parameters}

Various authors have established a relationship between the crystal formation, especially c-axis orientation in $\mathrm{ZnO}$ to the effect of the solvent [50] [64] stabilizer used in the sol-gel preparation [48], and the post deposition treatment applied [52]. However, while all these conditions, that is, solvent type, stabilizer used and post processing applied, were kept constant in this study, variations in the crystal growth process were still observed. This is an indication that the printing process parameters used for depositing the films, also, have an influence on the microstructural formation of the film.

The XRD spectra of the films printed with ink 2 using $100 \mu \mathrm{m}$ tip size with different pressures and writing speeds is shown in figures 5.6 and 5.7. The film shows a dominating crystal orientation in the 002 direction. However the intensity of the 002 peak increases as pressure increases, and reduces with speed. Films printed at 15 psi pressure and $1 \mathrm{~mm} / \mathrm{s}$ shows peak with higher intensity than that printed at $3 \mathrm{~mm} / \mathrm{s}$ and the same pressure (Figure 5.6). Also, the film printed with 20 psi pressure at 3 and $5 \mathrm{~mm} / \mathrm{s}$ (Figure 5.7) speed shows the same trend. Appearance of other $\mathrm{ZnO}$ peaks was observed at printing speed of $1 \mathrm{~mm} / \mathrm{s}$, however, the 002 still remains the dominating peak. Since, higher pressure and low speed favors the deposition of more materials, the observed behavior can be attributed to the quantity of material deposited in each parameter combination.

While using a larger tip size of $150 \mu \mathrm{m}$ to print ink 2 , the trend observed earlier still persists as only 002 peak was observed at printing conditions of 10 and $15 \mathrm{psi}$ and $3 \mathrm{~mm} / \mathrm{s}$. Increasing the pressure to $20 \mathrm{psi}$ at $3 \mathrm{~mm} / \mathrm{s}$ resulted in the formation of polycrystalline film with 100, 002 and 101 peaks with the 002 still the dominating peak (Figure 5.8). 


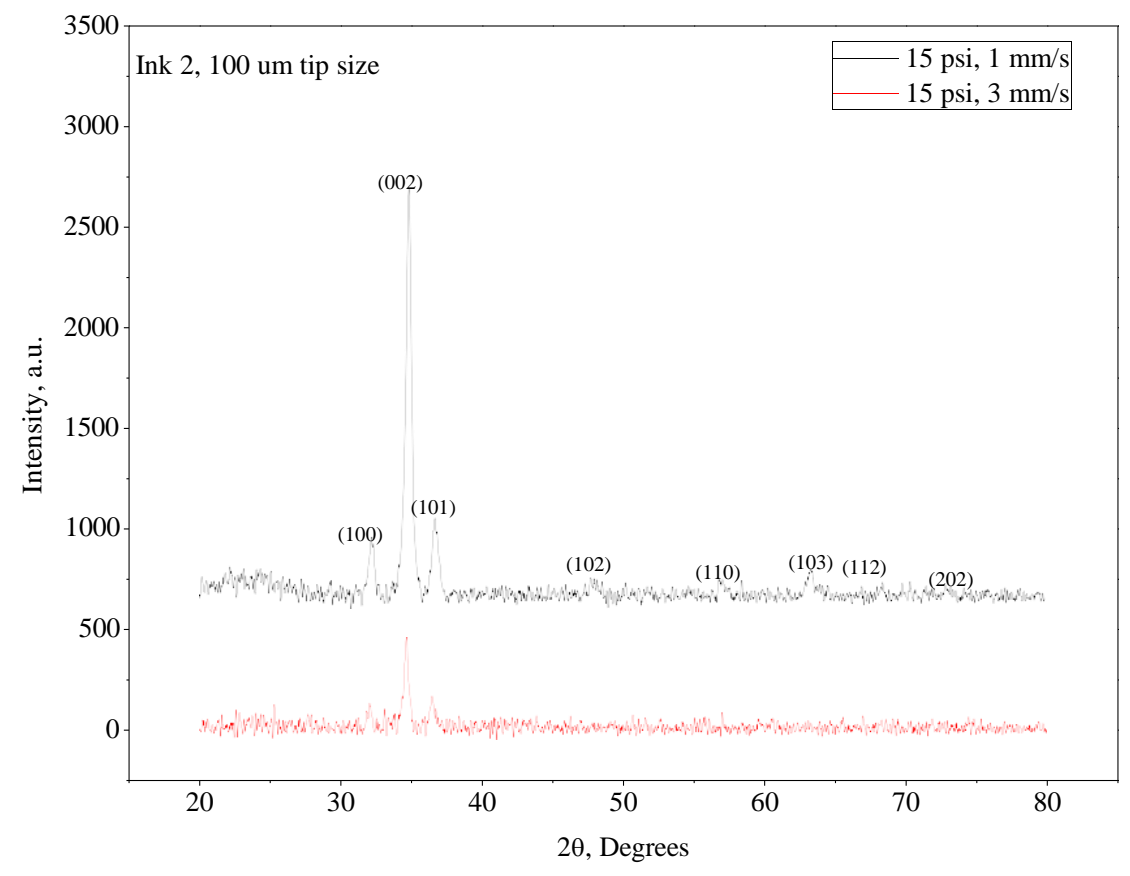

Figure 5.6 XRD spectrum of feature printed with ink 2, $100 \mu \mathrm{m}$ nozzle, 15 psi at speeds of 1 and $3 \mathrm{~mm} / \mathrm{s}$, and sintered at $500^{\circ} \mathrm{C}$ for 2 hours

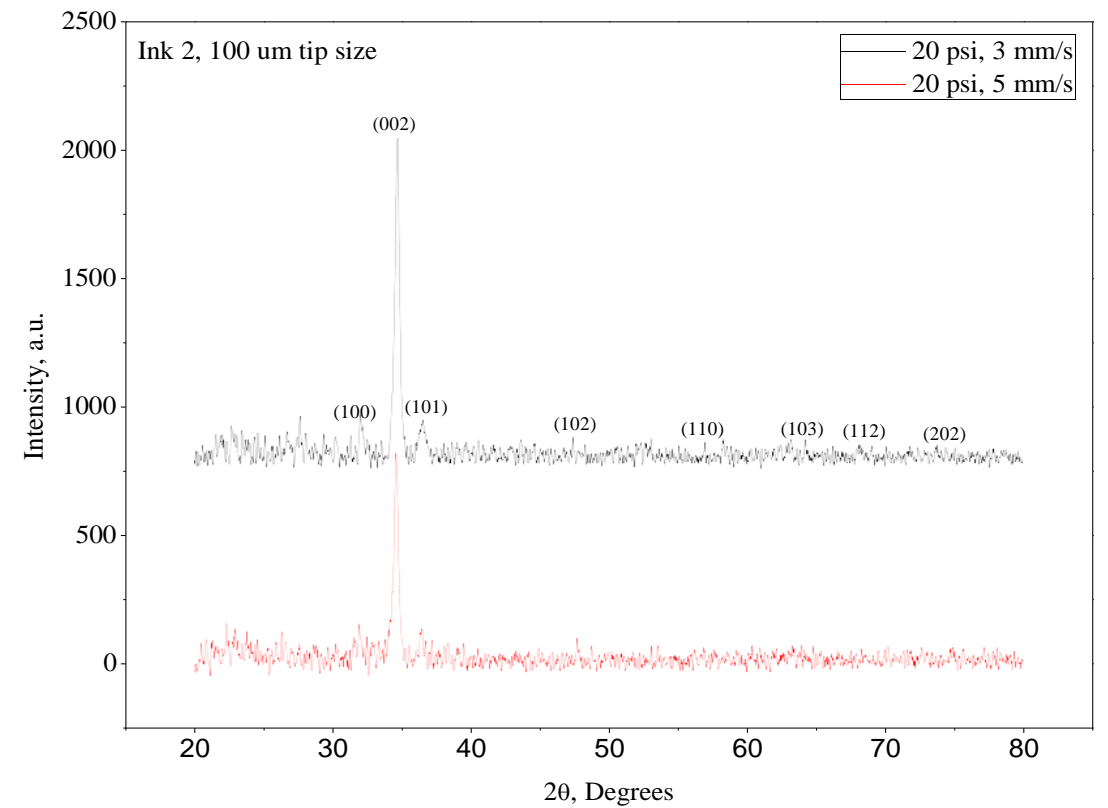

Figure 5.7 XRD spectrum of feature printed with ink $2,100 \mu \mathrm{m}$ nozzle, 20 psi at speeds of 3 and $5 \mathrm{~mm} / \mathrm{s}$, and sintered at $500^{\circ} \mathrm{C}$ for 2 hours 
However, a totally different crystal structure was obtained when the printing of ink 2 with $150 \mu \mathrm{m}$ nozzle was done at $15 \mathrm{psi}$ and $1 \mathrm{~mm} / \mathrm{s}$ (Figure 5.9). In this case, all $\mathrm{ZnO}$ peaks appeared and 101 peak becomes the dominating peak. At $200 \mu \mathrm{m}$ tip size, 100, 002 and 101 peaks appeared in all cases with 002 being the dominant peak (Figure 5.10). The deposition pressure and speed still play the role of either increasing or decreasing the intensity as earlier discussed.

As earlier mentioned, crystal structure of deposited films determines their potential applications. Therefore, the ability of achieving different crystal structures and orientations by changing printing parameters as observed in the results is of great interest. This capability provides us with a route that allows tailoring of deposited film to meet a particular purpose and application without necessarily altering the chemical composition or changing the post deposition treatment. However, to achieve this high level control on the crystal structure of film, in-depth understanding of the nucleation and growth of crystals as it applies to this printing process is required.

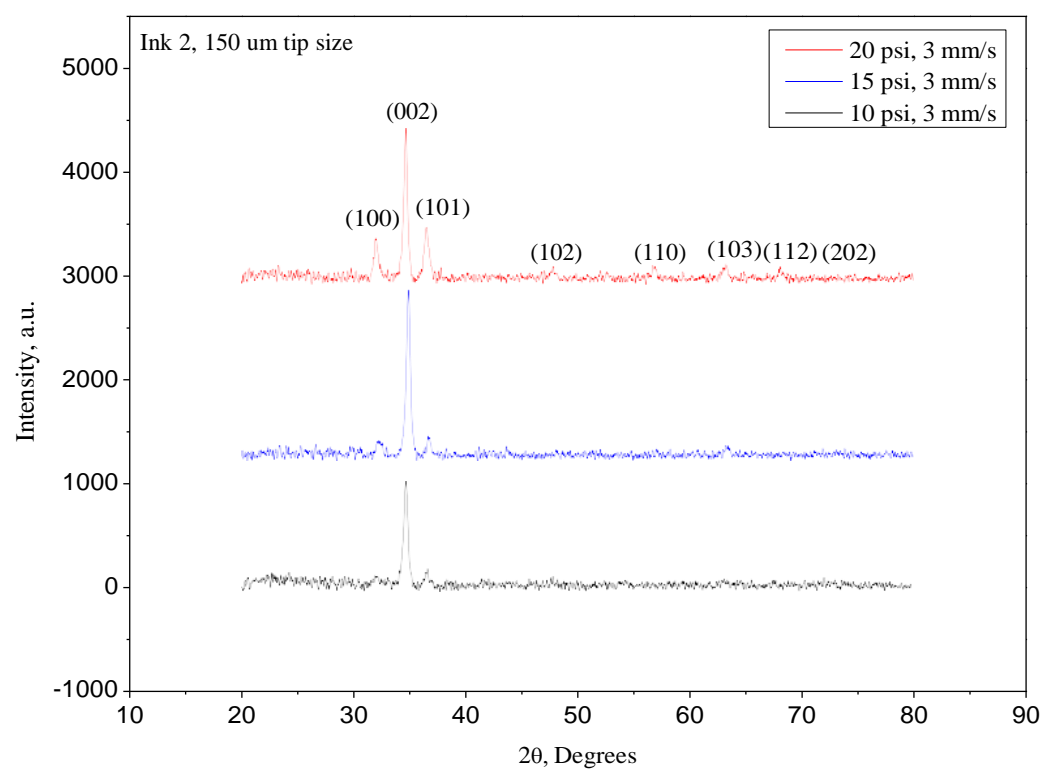

Figure 5.8 XRD spectrum of feature printed with ink $2,150 \mu \mathrm{m}$ nozzle, $3 \mathrm{~mm} / \mathrm{s}$ and 10,15 and 20 psi pressure, and sintered at $500^{\circ} \mathrm{C}$ for 2 hours 


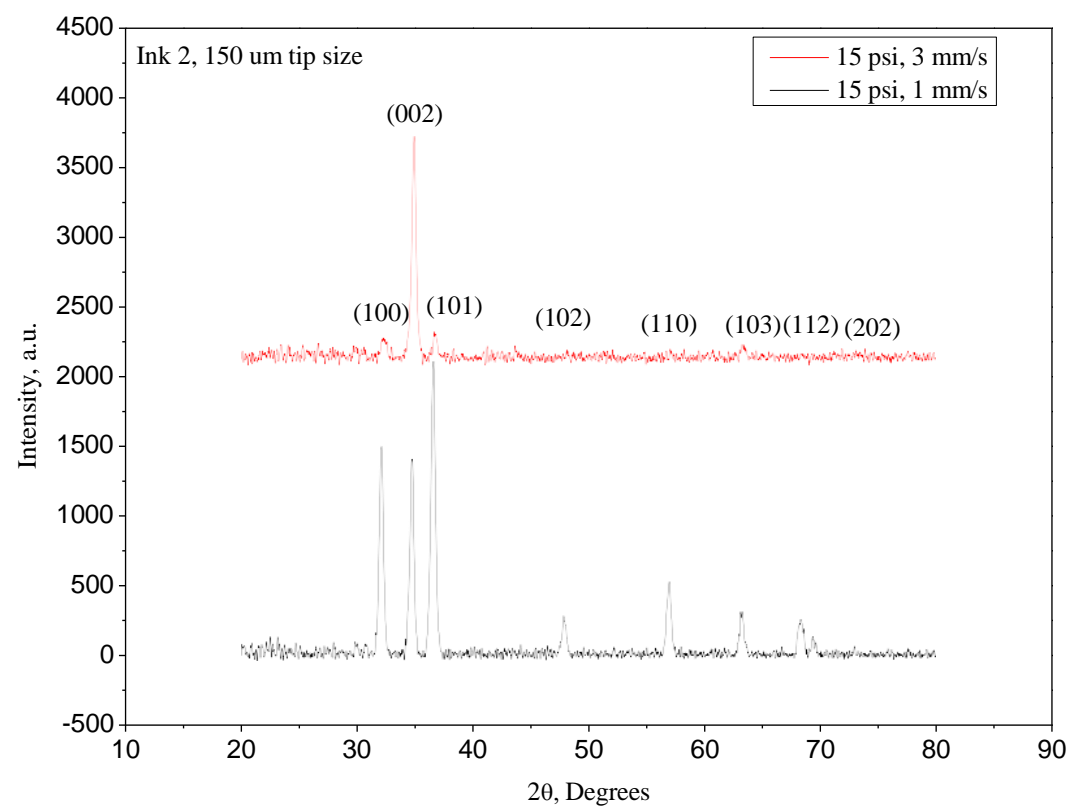

Figure 5.9 XRD spectrum of feature printed with ink 2, $150 \mu \mathrm{m}$ nozzle, 15 psi and 1 and 3 $\mathrm{mm} / \mathrm{s}$, and sintered at $500^{\circ} \mathrm{C}$ for 2 hours

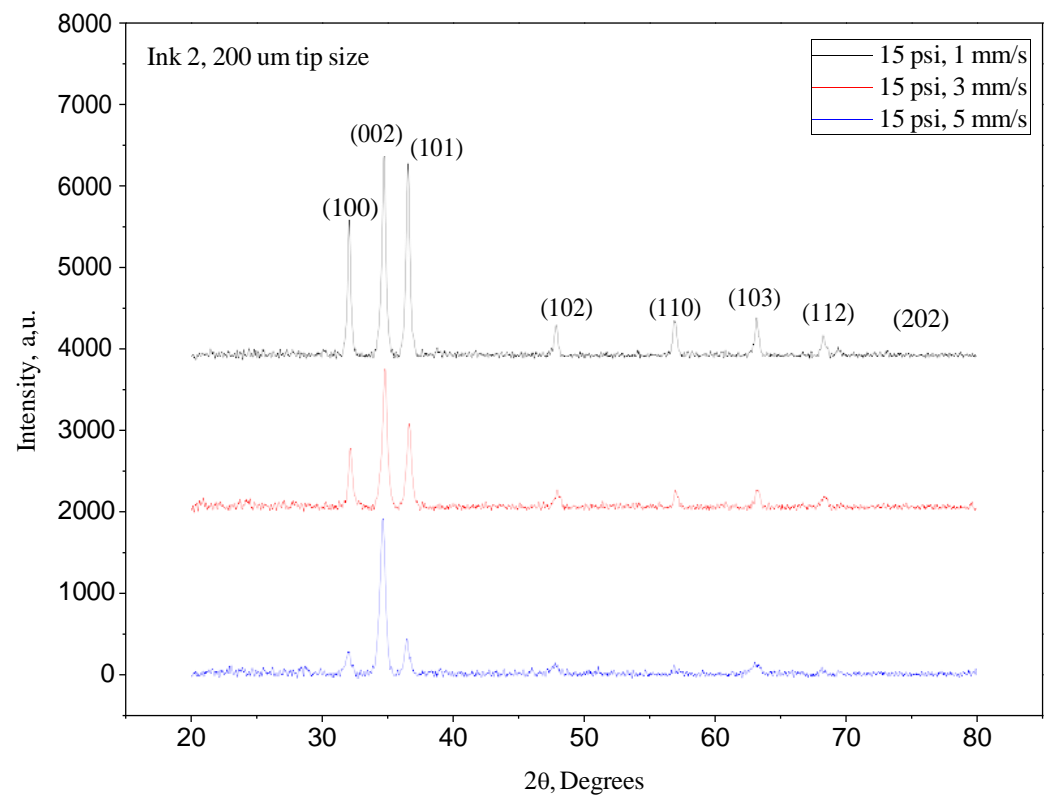

Figure 5.10 XRD spectrum of feature printed with ink 2, $200 \mu \mathrm{m}$ nozzle, 15 psi and 1, 3 and $5 \mathrm{~mm} / \mathrm{s}$, and sintered at $500^{\circ} \mathrm{C}$ for 2 hours 


\subsection{Crystal Growth Mechanism}

Various growth mechanisms have been put forward for $\mathrm{ZnO}$ thin films deposited through sol gel method by other researchers. Self-assembly based on dipole-dipole interaction between polar nano-grains was reported by Wang et al. [85] to be responsible for the c-axis orientation observed in $\mathrm{ZnO}$ film. Self-texture and self-template phenomenon were reported by Fujimura et al. [78] and Zhu et al., [85] respectively, to be the reason for the perceived crystal growth. All the factors reported thus far for the crystal growth are either extrinsic to the material (based on process parameters and chemical formation) or intrinsic to the material. The crystal structure of the directly written films was examined in this respect to determine the interplay of the extrinsic factors which are the printing parameters, in this case, and the intrinsic factors such as the self-texturing based on crystal interactions.

Printing ink 2 with $100 \mu \mathrm{m}$ tip at $20 \mathrm{psi}$ and $3 \mathrm{~mm} / \mathrm{s}$ yields a feature with $130 \mu \mathrm{m}$ in average width and $3.56 \mu \mathrm{m}$ height; printing at $15 \mathrm{psi}$ and $3 \mathrm{~mm} / \mathrm{s}$ produced feature with $133 \mu \mathrm{m}$ and 4.39 in height, and 169 and $4.75 \mu \mathrm{m}$ was obtained in width and height at a printing pressure of $20 \mathrm{psi}$ and $3 \mathrm{~mm} / \mathrm{s}$ speed (Figure 5.11 and 5.12). In all these cases, crystallization in c-axis orientation with sharp 002 peak were obtained while other peaks were either not present or of negligible intensity. Changing the printing parameters for ink 2 while using 100, 150, and $200 \mu \mathrm{m}$ tip size for printing, produced features with average width that ranged from 226 to $374 \mu \mathrm{m}$ and feature height ranging from 8.23 to $13.69 \mu \mathrm{m}$. Figure 5.12 to 5.14 show that crystal growth in this feature range was not totally confined to the c-axis direction as growth in 100 and 101 planes were observed. However, the 002 still remains the dominating peak. Further changes in printing parameters to produce feature with widths from 486 to $796 \mu \mathrm{m}$ and 15 to $22 \mu \mathrm{m}$ in height, while still using ink 2 and 150 or $200 \mu \mathrm{m}$ nozzle was adopted. Here, appearance of almost all the $\mathrm{ZnO}$ 
wurtzite peaks were noticed which shows the polycrystalline nature of the films obtained. The 100, 002 and 101 peaks were the major peaks observed, and the three peaks were comparable in terms of intensity (figures 5.13 and 5.14).

From the aforementioned it appears there is a threshold width at which transition from nano-crystallinity to poly-crystallinity occurs. Films with widths between 50 and $200 \mu \mathrm{m}$ have only 002 peak, indicating crystal growth in c-axis alone, while those with widths between 200 and $400 \mu \mathrm{m}$ grows in 100, 002 and 101 directions, but the growth in 100 and 101 directions were not enough to catch up with the 002 direction due to material shortage. Those with widths between 400 and $900 \mu \mathrm{m}$ produced films with crystal growth in different directions with the 100 and 101 peaks becoming comparable with 002 peak in intensity, as enough materials were available to support growth in the high surface energy planes. This observation is schematically shown in figure 5.15 .

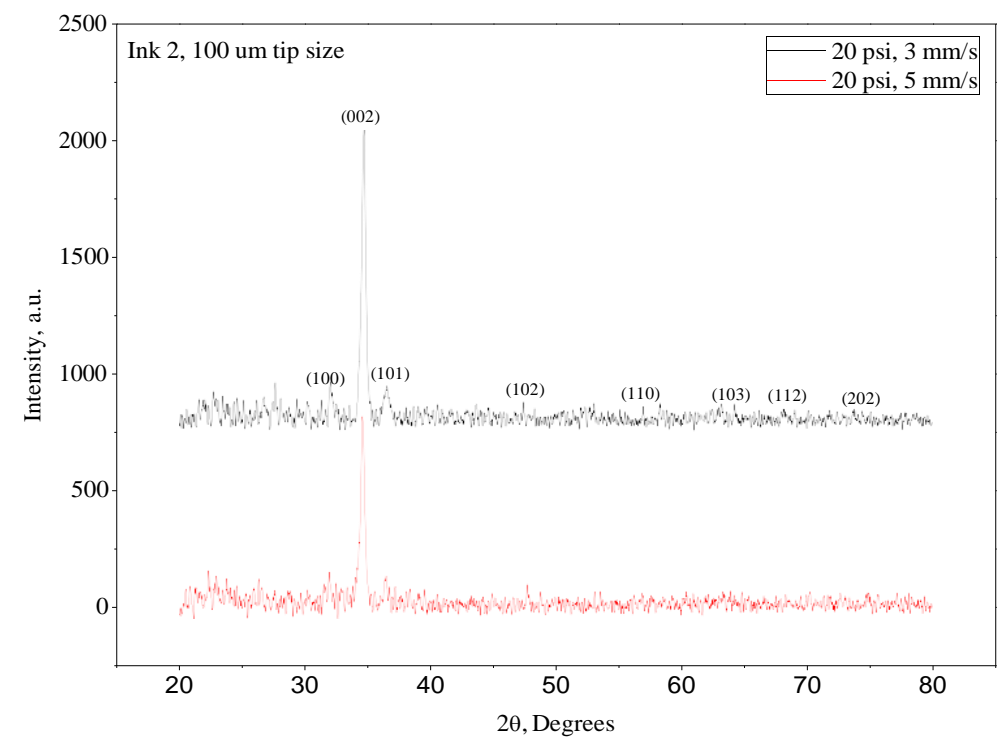

Figure 5.11 XRD spectrum of feature printed with ink 2, $100 \mu \mathrm{m}$ nozzle, 20 psi at 3 and 5 $\mathrm{mm} / \mathrm{s}$, and sintered at $500^{\circ} \mathrm{C}$ for 2 hours 


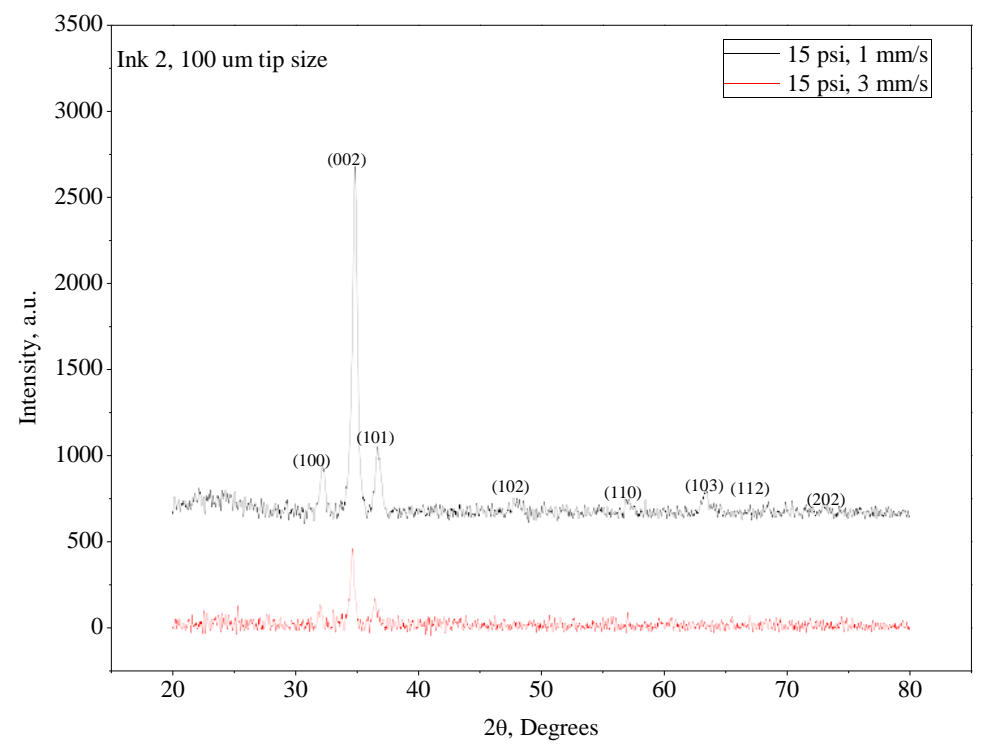

Figure 5.12 XRD spectrum of feature printed with ink 2, $100 \mu \mathrm{m}$ nozzle, 15 psi at 1 and 3 $\mathrm{mm} / \mathrm{s}$, and sintered at $5_{00}^{\circ} \mathrm{C}$ for 2 hours

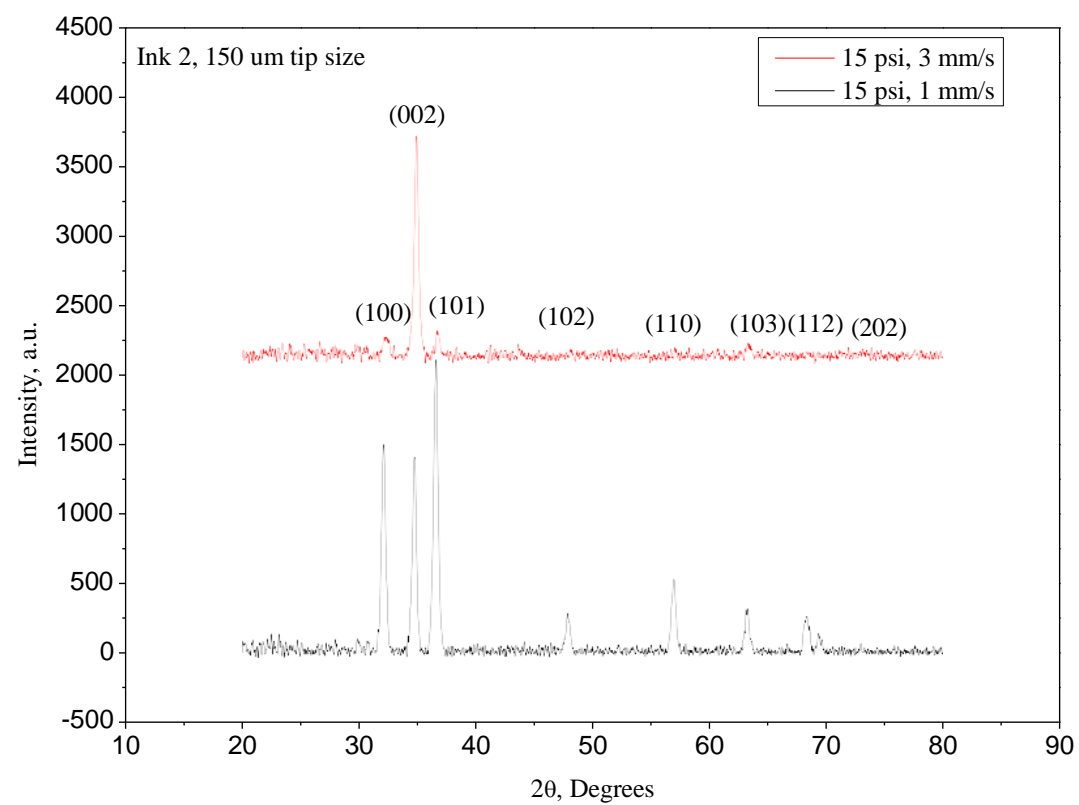

Figure 5.13 XRD spectrum of feature printed with ink 2, $150 \mu \mathrm{m}$ nozzle, 15 psi at 1 and 3 $\mathrm{mm} / \mathrm{s}$, and sintered at $500^{\circ} \mathrm{C}$ for 2 hours 


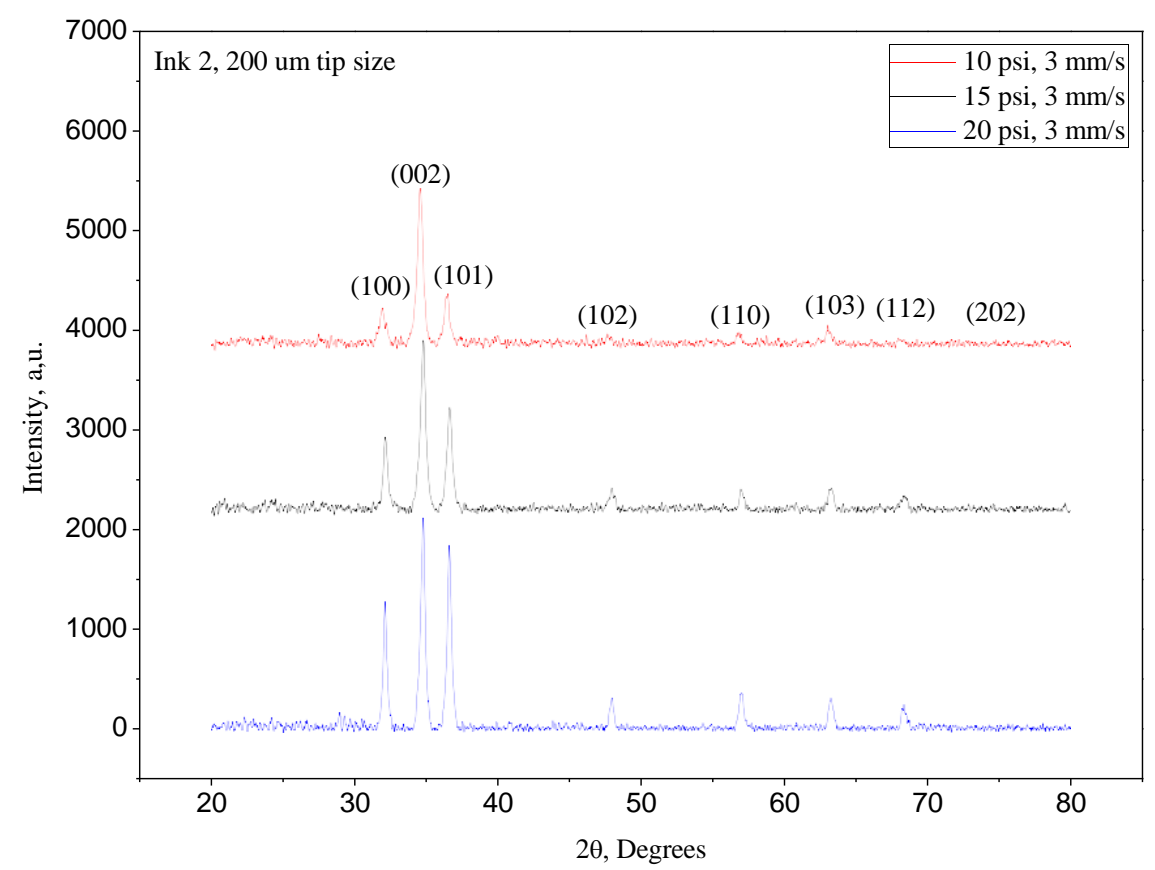

Figure 5.14 XRD spectrum of feature printed with ink 2, $200 \mu \mathrm{m}$ nozzle, $3 \mathrm{~mm} / \mathrm{s}$ at 10, 15 and $20 \mathrm{psi}$, and sintered at $500^{\circ} \mathrm{C}$ for 2 hours

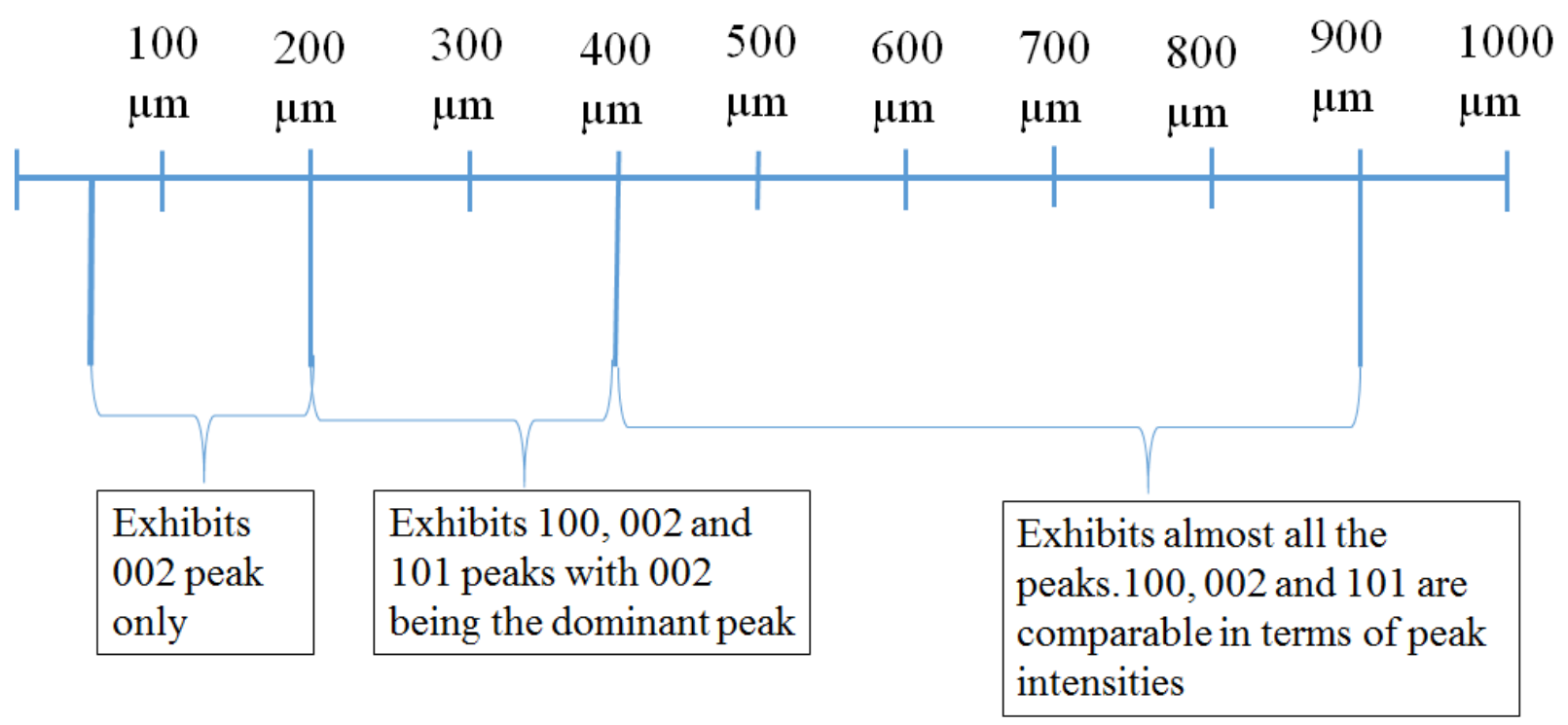

Figure 5.15 Schematic of the observed crystal orientation at different feature widths 
Based on this hypothesis, films printed from different inks (inks 1 and 3) with feature widths in the ranges defined above were examined for their crystal structure. It was found that the theory holds, as the films with widths less than 200, between 200 and $400 \mu \mathrm{m}$, and greater than $400 \mu \mathrm{m}$ shows similar behavior to that observed for ink 2 (Figures 5.16 to 5.19). In light of this discovery, the mechanism of crystal growth for film printed with NBDW can be conceptualized as a combination of both the extrinsic and intrinsic factors. The extrinsic factors create the environment and the limits, while the intrinsic determines crystal orientation and size in accordance with the set limits. Probable explanation of the crystal growth mechanism observed for the printed film is therefore given, taking the boundaries into consideration.

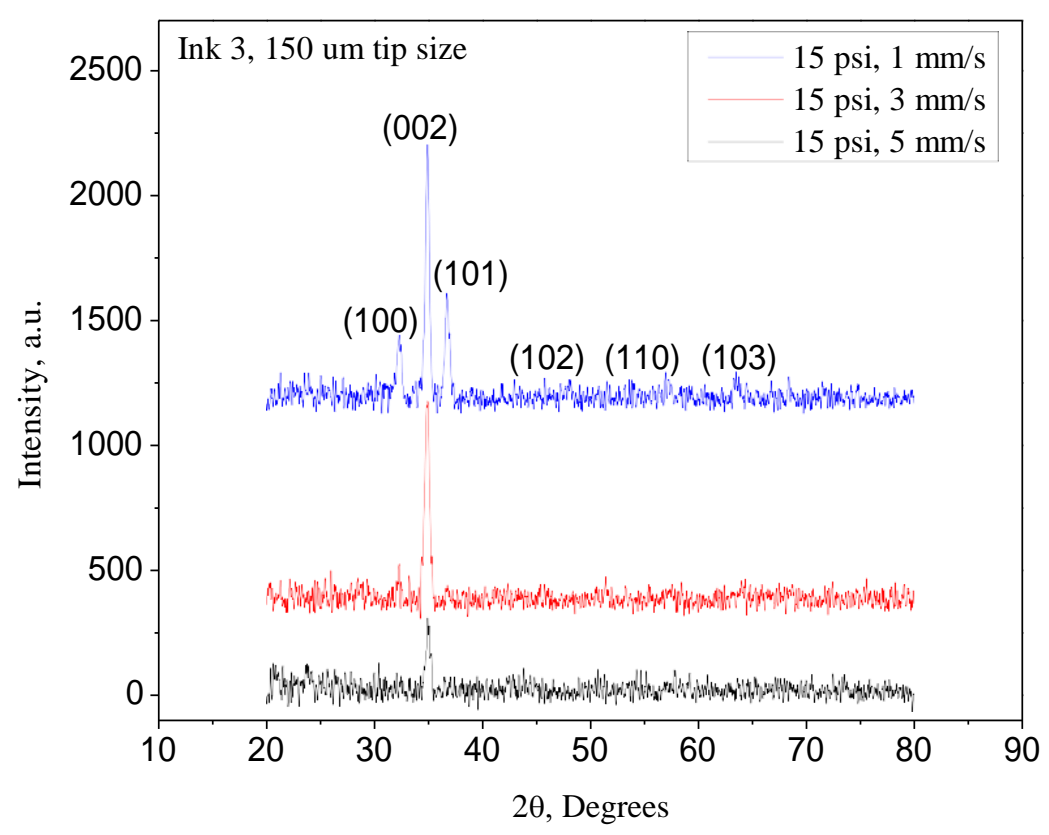

Figure 5.16 XRD spectrum of feature printed with ink 3, $150 \mu \mathrm{m}$ nozzle, 15 psi 1,3 and 5 $\mathrm{mm} / \mathrm{s}$, and sintered at $5^{\circ} 0^{\circ} \mathrm{C}$ for 2 hours 


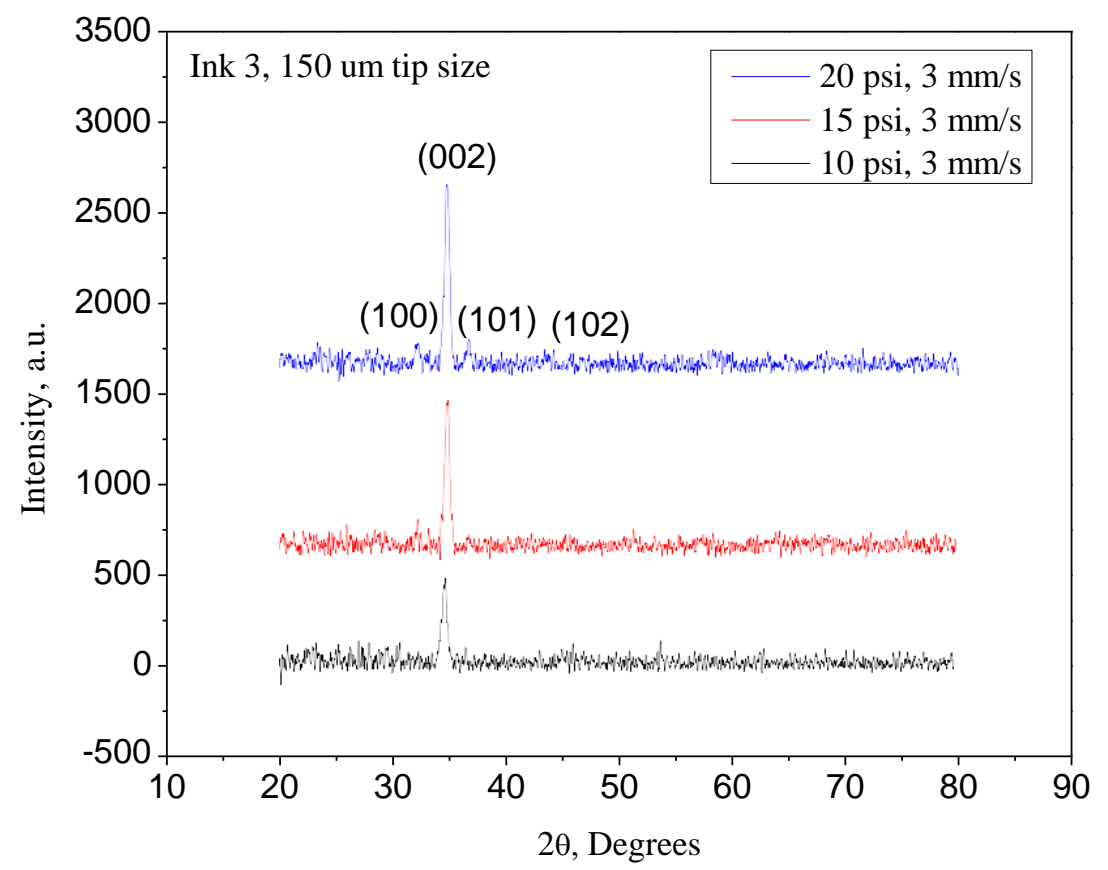

Figure 5.17 XRD spectrum of feature printed with ink $3,150 \mu \mathrm{m}$ nozzle, $3 \mathrm{~mm} / \mathrm{s}$ at 10,15 and $20 \mathrm{psi}$, and sintered at $500^{\circ} \mathrm{C}$ for 2 hours

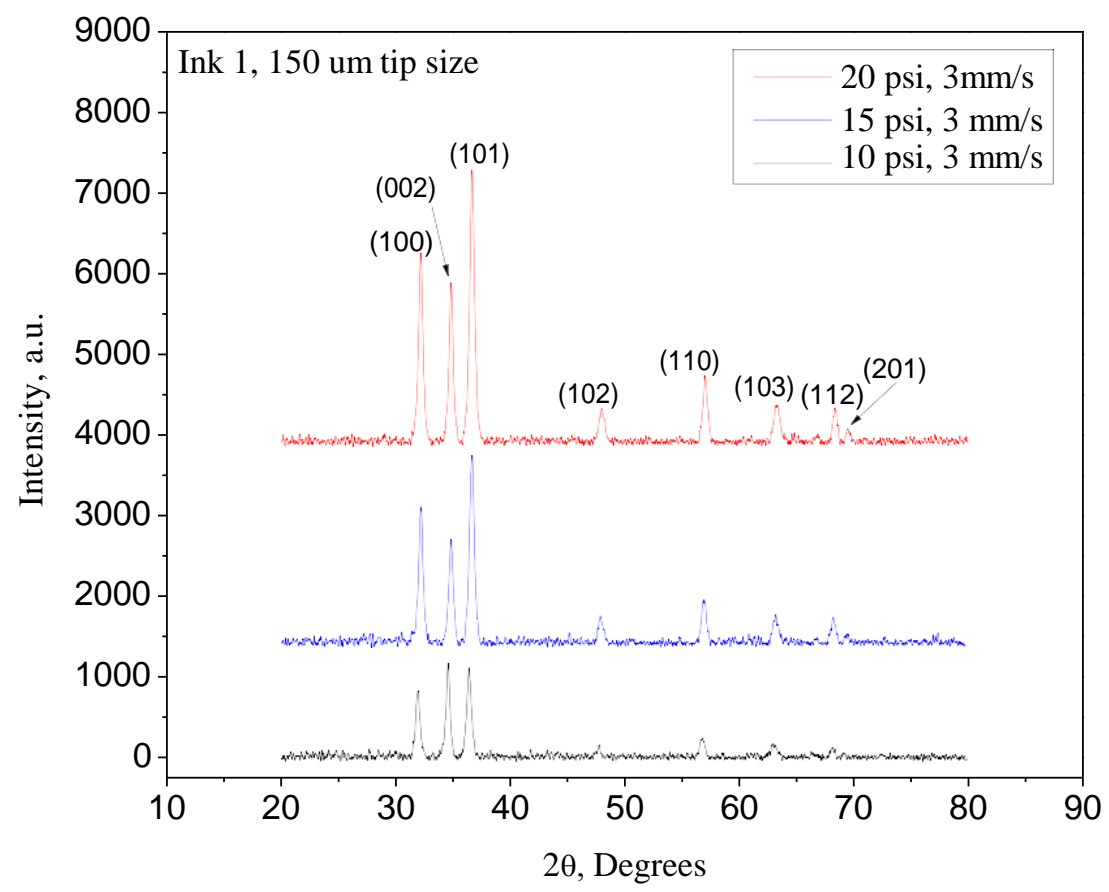

Figure 5.18 XRD spectrum of feature printed with ink $1,150 \mu \mathrm{m}$ nozzle, $3 \mathrm{~mm} / \mathrm{s}$ at 10,15 and $20 \mathrm{psi}$, and sintered at $500^{\circ} \mathrm{C}$ for 2 hours 


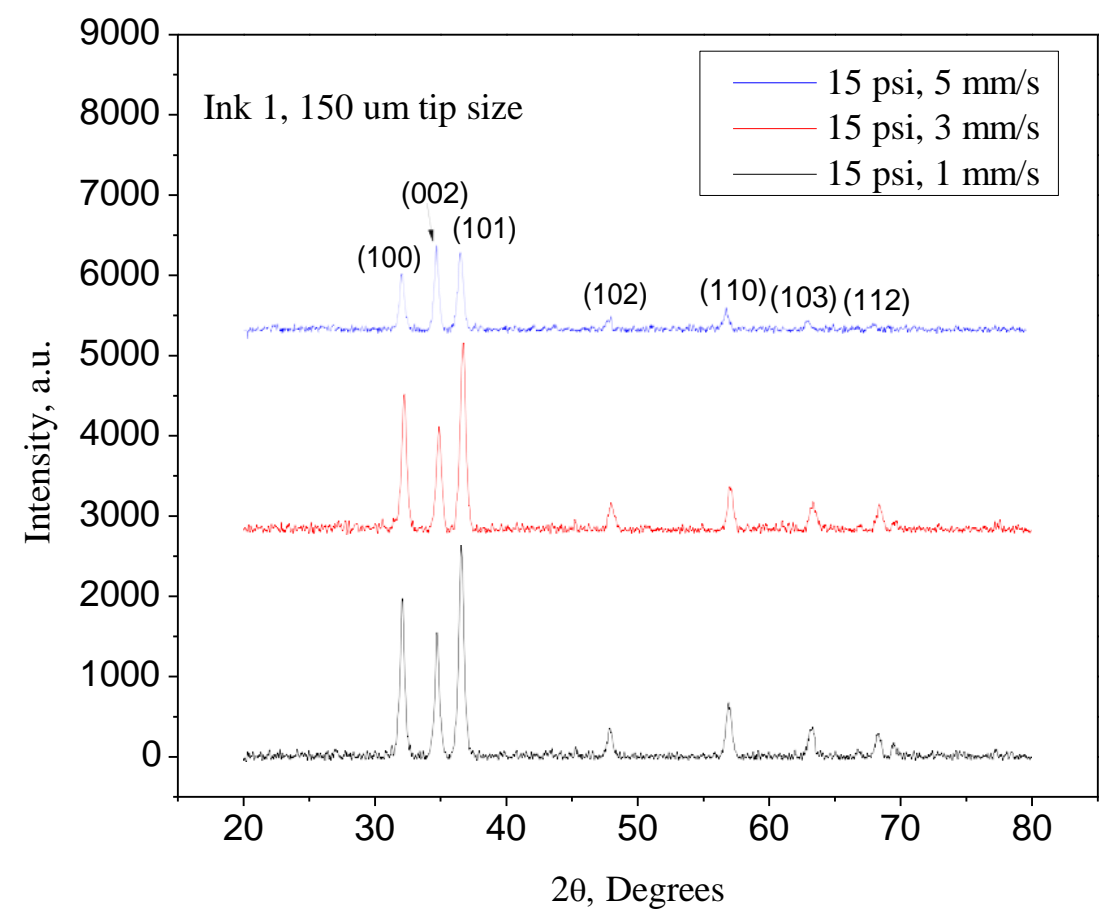

Figure 5.19 XRD spectrum of feature printed with ink $2,200 \mu \mathrm{m}$ nozzle, $3 \mathrm{~mm} / \mathrm{s}$ at 10,15 and $20 \mathrm{psi}$, and sintered at $500^{\circ} \mathrm{C}$ for 2 hours

After deposition, there is substrate-crystal and crystal-crystal interactions. However, for films deposited on glass substrate, no epitaxial relationship exists between $\mathrm{ZnO}$ and the substrate since glass is an amorphous material. During sintering, formation of nuclei takes place at various locations within the film and then grows into crystals. The film with larger width and height has smaller grain sizes indicating that many nuclei were formed. During crystal growth, contacts among the grains prevent further growth into larger sizes. While those with smaller width and consequently less material had a few nucleation points, thus, have enough space to grow into bigger crystals. This is also observed in the SEM micrographs shown in figures 5.20 and 5.21, where, deposition of more material produced higher density film. 


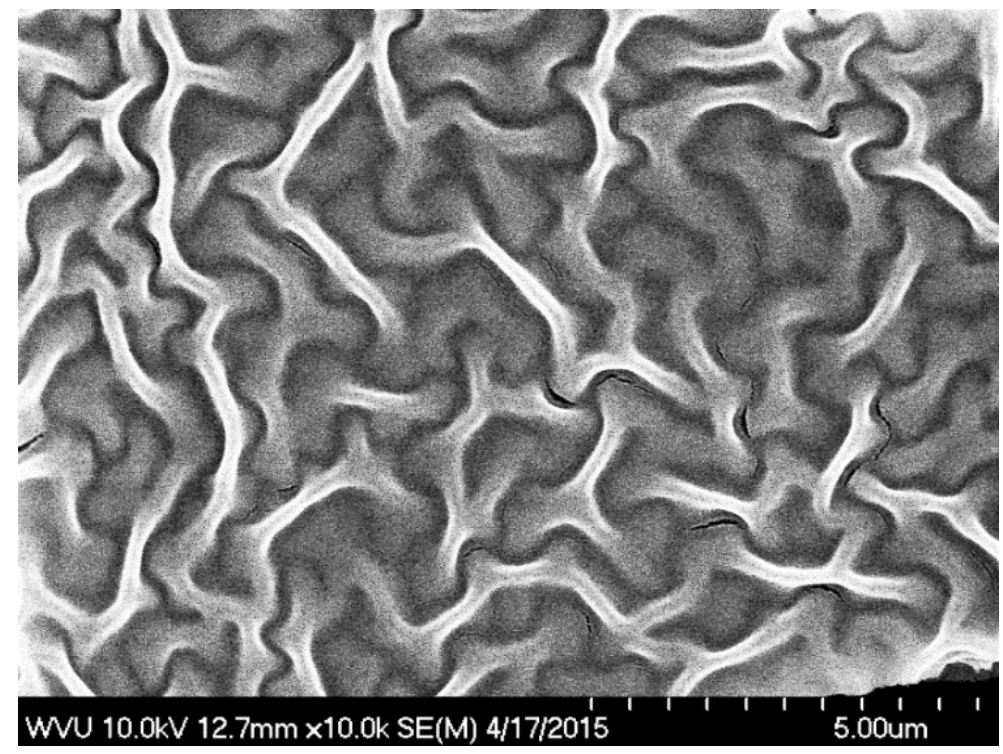

Figure 5.20 SEM image for ink 2 printed with $100 \mu \mathrm{m}$ nozzle, $15 \mathrm{psi}$ and $3 \mathrm{~mm} / \mathrm{s}$, and sintered at $500^{\circ} \mathrm{C}$ for 2 hours

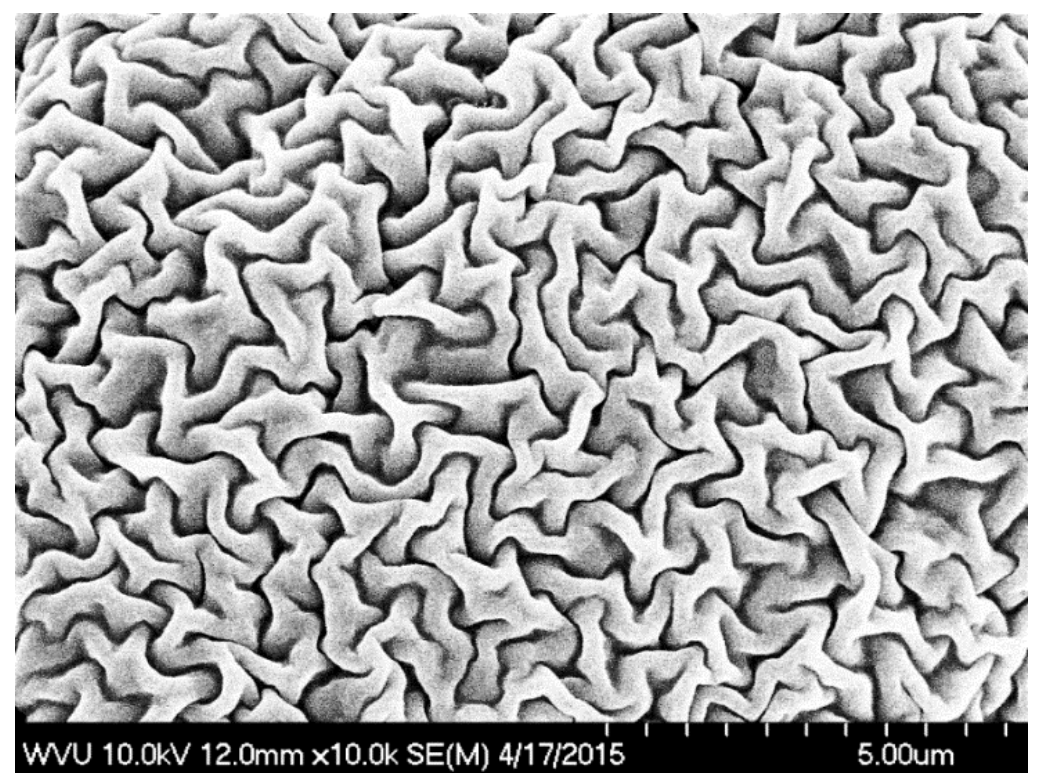

Figure 5.21 SEM image for ink 2 printed with $100 \mu \mathrm{m}$ nozzle, $15 \mathrm{psi}$ and $3 \mathrm{~mm} / \mathrm{s}$, and sintered at $500^{\circ} \mathrm{C}$ for 2 hours 
Since fewer nuclei were formed in the smaller width films, the chosen growth direction is the direction with minimum surface free energy where least resistance to crystal growth is encountered. This direction corresponds to the 002 direction in $\mathrm{ZnO}$ [78]. This explains why only 002 peak was present in films with widths less than $200 \mu \mathrm{m}$. On the other hand, presence of many nuclei in the films with larger width, results in growth in 002 direction at first, due to it being the lowest surface energy axis. As the growth continues, crystal-crystal interaction occurs and some crystals are forced to grow in other directions. The next low energy directions after 002, which are 100 and 101 are chosen by the crystals. If the available material is enough for crystallization in all directions (width greater than $400 \mu \mathrm{m}$ ), then all the $\mathrm{ZnO}$ peaks will be seen, else, the three minimum energy directions are occupied.

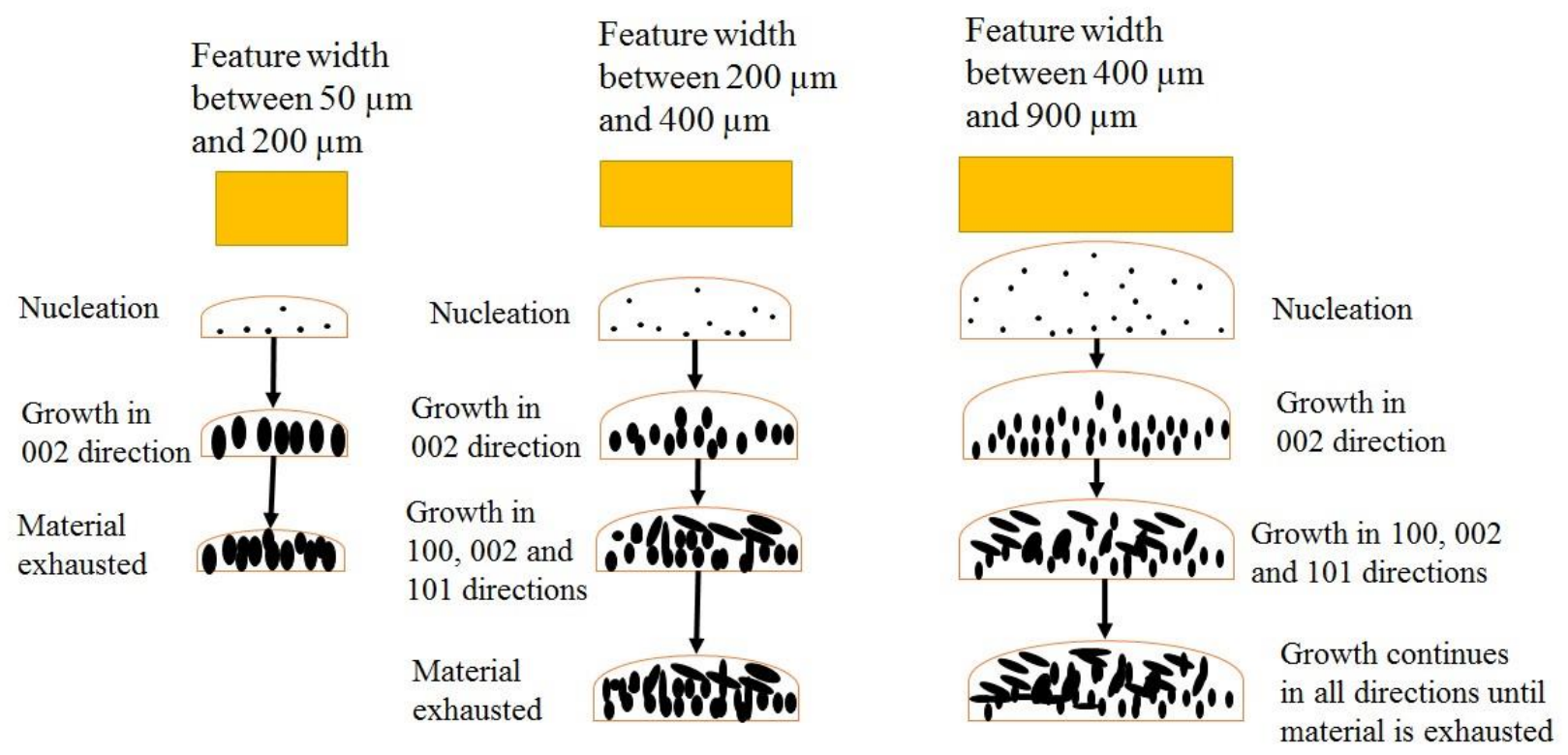

Figure 5.22 Schematic representation of perceived crystal nucleation and growth mechanism 


\section{ELECTRICAL CHARACTERIZATION OF DIRECTLY WRITTEN PATTERNS}

\subsection{Effect of Printing Conditions on Film Conductivity}

Conductivity of $\mathrm{ZnO}$ thin film constitutes an important step toward the development of electronic devices based on oxide thin films or nanostructures. The current-voltage (I-V) characteristics of AZO films deposited on glass substrate at various printing conditions and sintered at $500^{\circ} \mathrm{C}$ for 2 hours for some selected printing conditions were measured using a bias voltage of -5 to $+5 \mathrm{~V}$. The I-V relationship observed, are found to be symmetric and linear with forward and reverse bias indicating the existence of an ohmic contact at the Silver-AZO interfaces. Figure 6.1 shows the I-V curve obtained for pattern printed with ink 1 , using $150 \mu \mathrm{m}$ nozzle and 10 psi at different writing speeds. Ohmic relationship was exhibited in all cases, maximum current, which corresponds to minimum resistance was obtained at writing speed of $3 \mathrm{~mm} / \mathrm{s}$, giving approximately $7 \times 10^{-6} \mathrm{~A}$ at bias voltage of $5 \mathrm{~V}$. While printing with ink 2 (Figure 6.2), using 150 $\mu \mathrm{m}$ nozzle and 20 psi pressure at different writing speeds. It was observed that the film conductivity decreases with increase in speed for the pattern printed at $1 \mathrm{~mm} / \mathrm{s}$ having a maximum current of approximately $7.58 \times 10^{-7} \mathrm{~A}$ at bias voltage of $5 \mathrm{~V}$. These values are higher than the dark current reported for $\mathrm{ZnO}$ nanowire by $\mathrm{Bao}$ et al. [70] and values reported for pulsed laser deposited Magnesium doped $\mathrm{ZnO}$ reported by Shewale et al., [55]. Ink 3 (Figure 6.3) also show similar behavior as ink 1, with the pattern printed at $3 \mathrm{~mm} / \mathrm{s}$ having the highest conductivity.

This observed trend in the conductivity of the deposited AZO films requires a critical assessment to be able to understand factors responsible for this behavior. Since resistivity is a function of area, it is expected that materials printed at lower speeds with larger width should have 
higher conductivity as observed for ink 2 (Figure 6.2). The decrease in conductivity observed for $1 \mathrm{~mm} / \mathrm{s}$ speed as compared with that of $3 \mathrm{~mm} / \mathrm{s}$ for ink 1 can be attributed to presence of too many grain boundaries formed as a result of excessive deposition of materials. Due to large volume of materials deposited, cracks are initiated at the grain boundaries leading to electrons being trapped and thus causing hindrance to the flow of current.

It was also observed that the resistance to the flow of current is higher for ink 3 than for ink 2 and ink 1 in all cases. This can be due to the fact that the PVP used in tuning the inks' viscosity is burnt off during sintering, thus, making films printed from high polymer content inks more porous and consequently, more resistant to flow of current. This is probably the reason for the trend observed in ink 3 where the pattern printed at $1 \mathrm{~mm} / \mathrm{s}$ has lower conductivity than that of $3 \mathrm{~mm} / \mathrm{s}$.

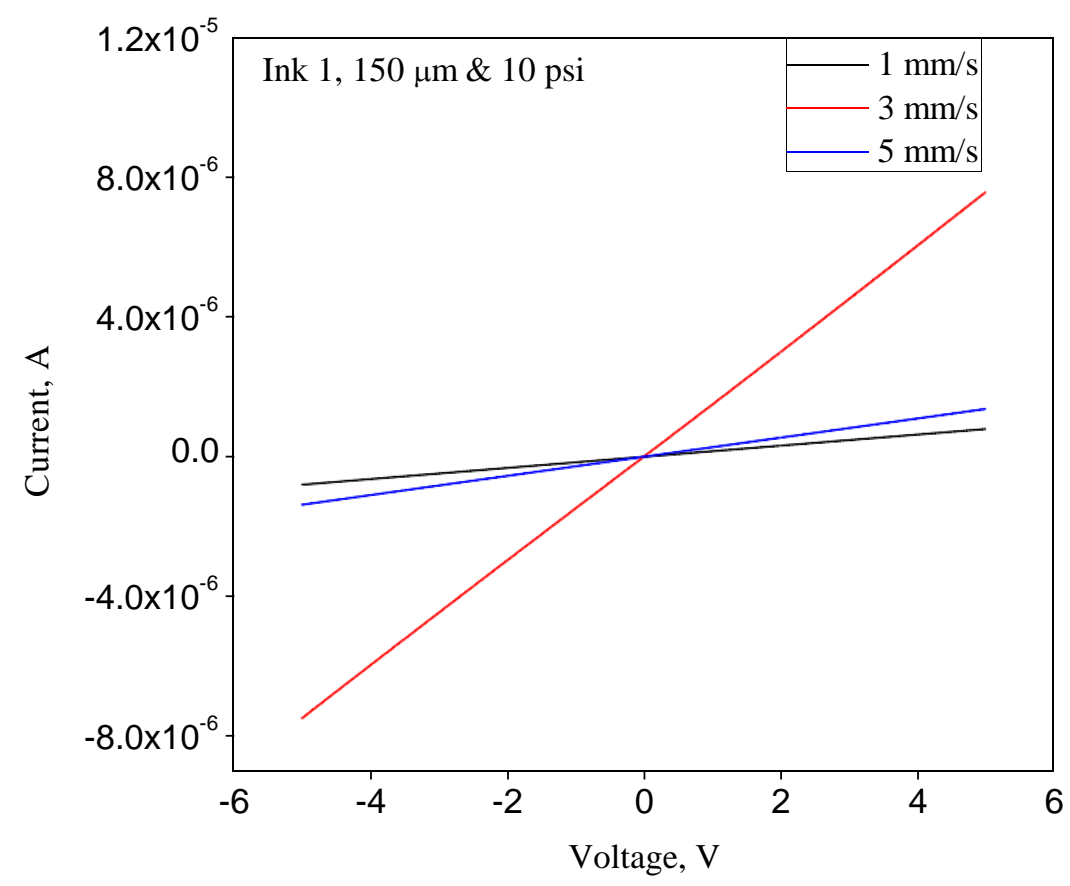

Figure 6.1 Current versus voltage for pattern printed with ink $1,150 \mu \mathrm{m}$ nozzle and $10 \mathrm{psi}$ pressure at different speeds 


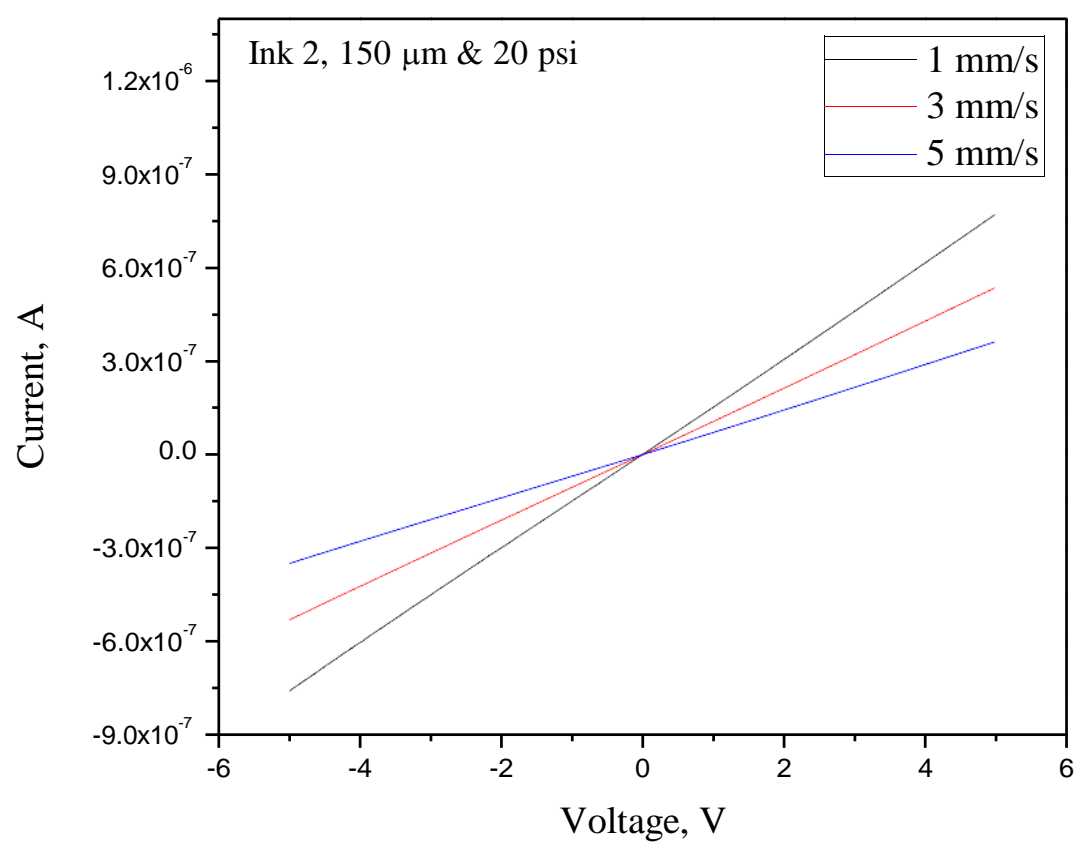

Figure 6.2 Current versus voltage for pattern printed with ink $2,150 \mu \mathrm{m}$ nozzle and 20 psi pressure at different speeds

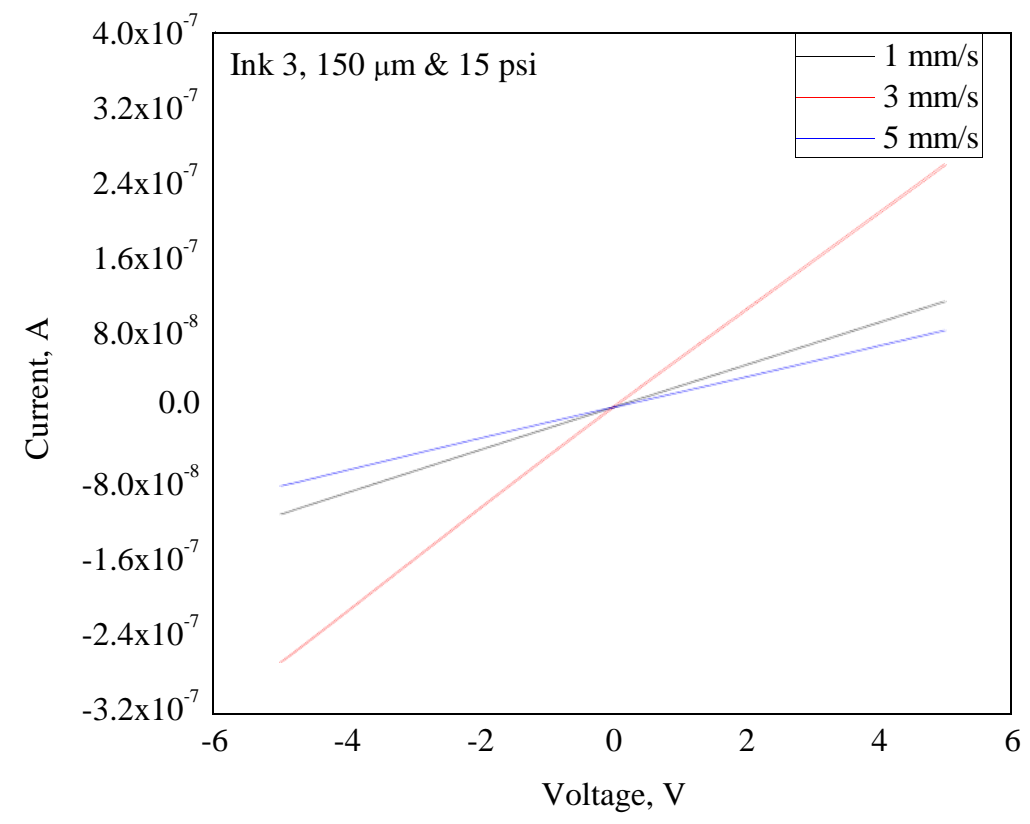

Figure 6.3 Current versus voltage for pattern printed with ink 3, $150 \mu \mathrm{m}$ nozzle and 15 psi pressure at different speeds 


\subsection{Photoconductivity of Printed AZO Films}

Illumination of $\mathrm{ZnO}$ surface with UV light of energy higher than its band gap has been reported to increase the conductivity of the material as more holes and electrons are freed by the excitation process [55] [66] [71]. Figure 6.4 shows the response of film printed with ink 1 using $150 \mu \mathrm{m}$ nozzle and 10 psi pressure at $3 \mathrm{~mm} / \mathrm{s}$ speed. Two UV intensities tagged UV1 and UV2 obtained by powering $365 \mathrm{~nm}$ UV lamp with 4.5 and $6 \mathrm{~V}$, respectively. The conductivity of the film increased by upto twenty times, upon UV illumination compared to what was obtained without UV exposure. Conductivity was also found to increase further with increase in UV intensity, indicating that, the depth reached by UV light to free electrons depends on its intensity. Similar behavior was also exhibited by ink 2, shown in figure 6.5 as its resistance decreases from $6.01 \times 10^{6} \Omega$ at unexcited state to $2.18 \times 10^{5} \Omega$ upon excitation with $\mathrm{UV} 1$ and to $1.98 \times 10^{5} \Omega$ when excited with UV2.

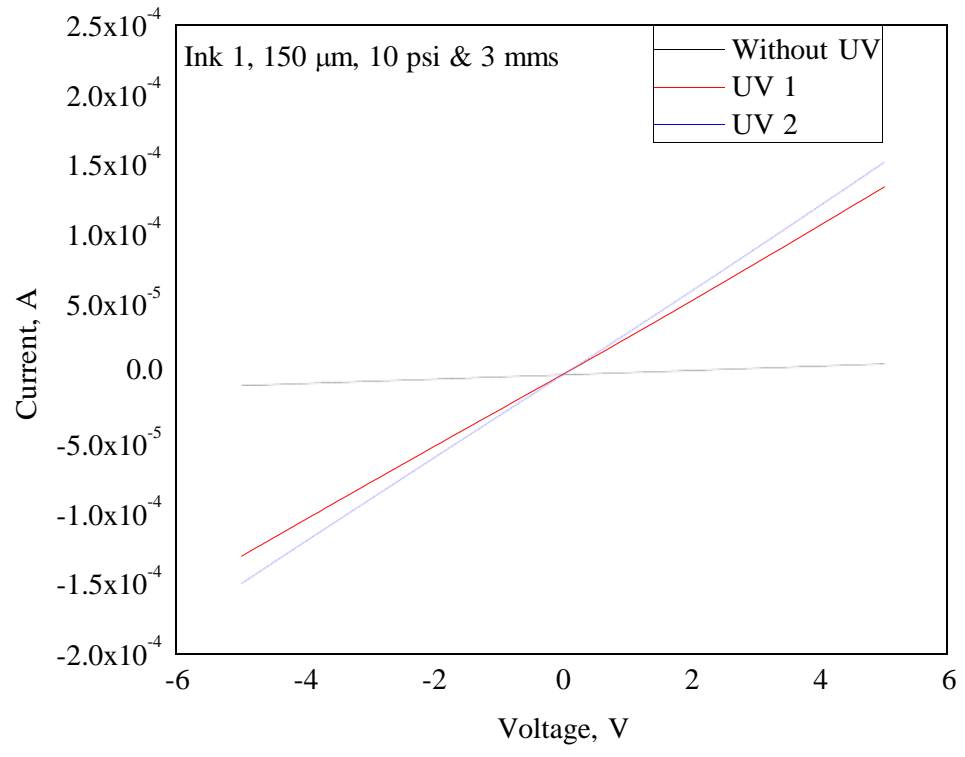

Figure 6.4 Current versus voltage for pattern printed with ink $1,150 \mu \mathrm{m}$ nozzle, 10 psi and $3 \mathrm{~mm} / \mathrm{s}$, with and without $\mathrm{UV}$ excitation 


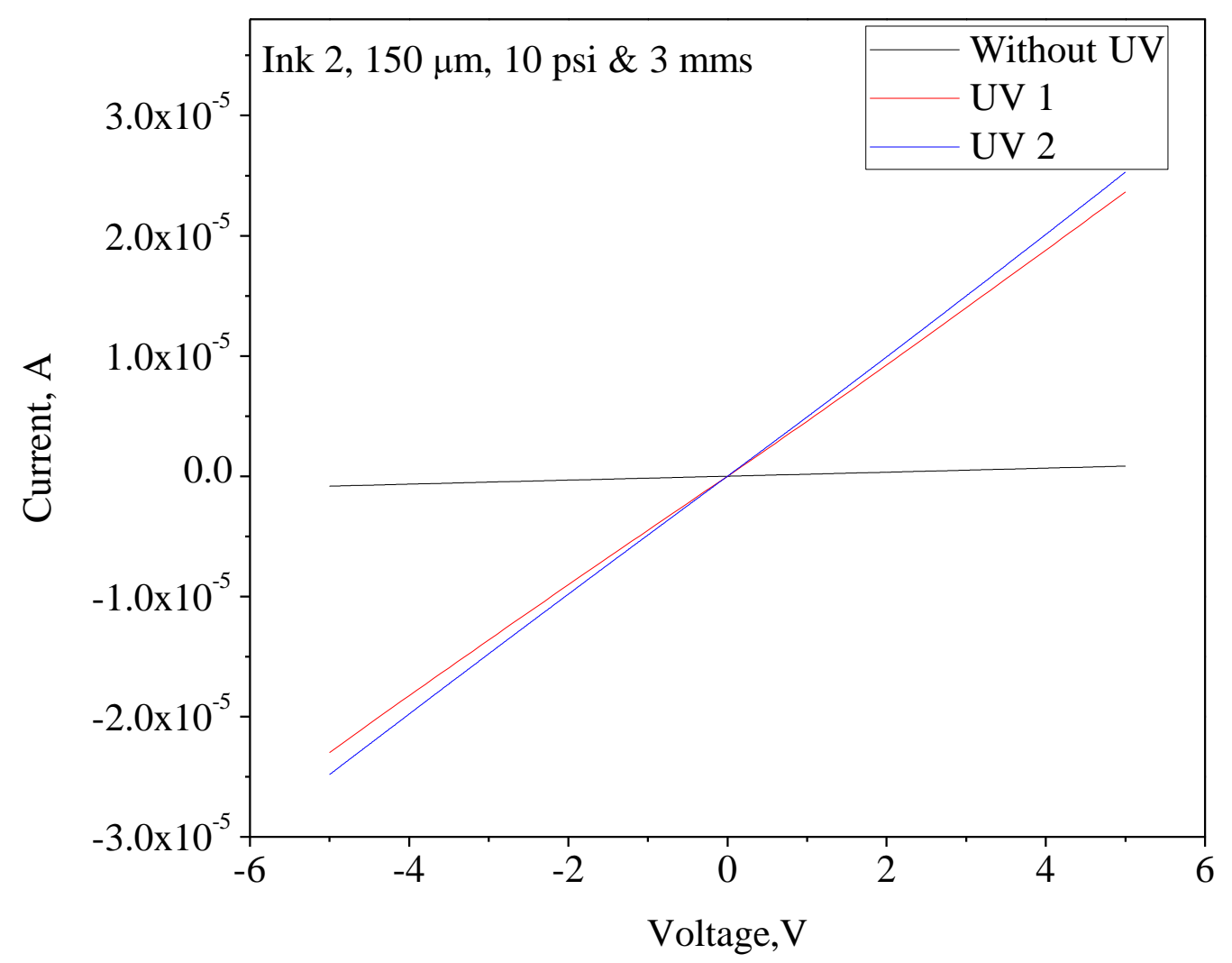

Figure 6.5 Current versus voltage for pattern printed with ink $2,150 \mu \mathrm{m}$ nozzle, 10 psi and $3 \mathrm{~mm} / \mathrm{s}$, with and without UV excitation

The resistance of a multilayer deposited AZO film was measured at different temperatures under UV illumination. Figure 6.6 depicts the plot of resistance versus temperature for different UV illumination. It is observed that at all temperatures, the UV irradiated sample showed lower resistance compared to the one without UV exposure. For no UV exposure, the resistance of the material decreases continuously with increase in temperature. This is believed to be due to the energy received by the electrons which results in them gaining enough kinetic energy to tunnel through the grain boundaries. Increasing the temperature under UV illumination from 25 and $50^{\circ} \mathrm{C}$, results in a slight decrease in resistance. However, increasing the temperature to 75 and $100^{\circ} \mathrm{C}$ do not show any marked difference in the observed resistance. 


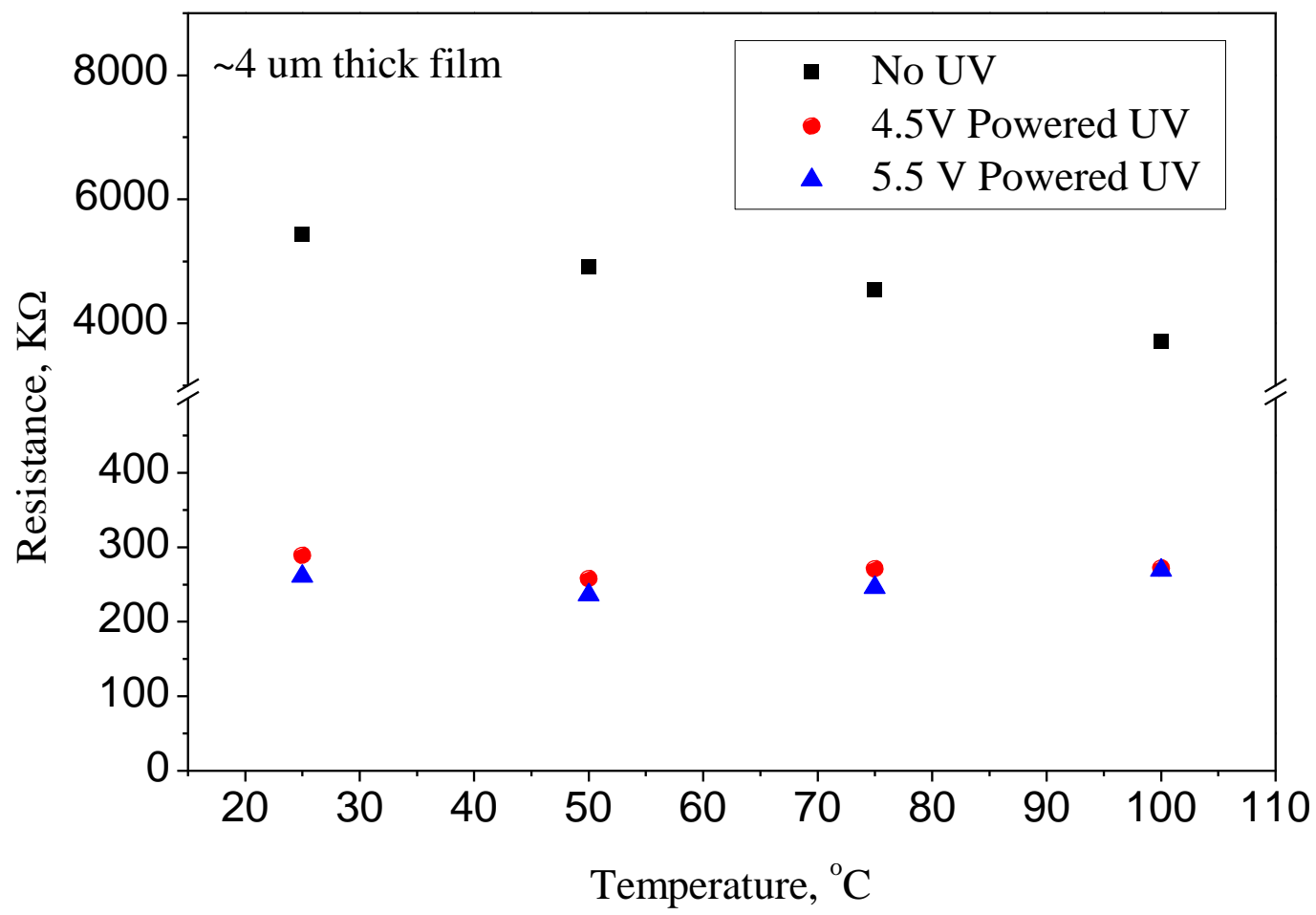

Figure 6.6 Resistance versus temperature for dark and UV irradiated films

Figures 6.7 to 6.9 shows the response over an extended period of time for film printed with ink 2, 15 psi pressure and $3 \mathrm{~mm} / \mathrm{s}$ at different temperatures and illuminated with $365 \mathrm{~nm}$ UV light of various intensities. UV1, UV2 and UV3 in this case refers to powering the UV lamp with 3.5, .5 and $5.5 \mathrm{~V}$, respectively. For all temperatures, sharp decrease in resistance is observed when the UV light is switched on or the intensity is increased, signifying further desorption of adsorbed oxygen. A reverse process was observed when the UV light was switched off, the resistance increased rapidly as a result of re-adsorption of oxygen from the atmosphere and causing electrons to be trapped again. It is however, observed that this relaxation time varies for different temperatures. The relaxation time decreases with increase in temperature. This observation can be observed when relaxation time at $25^{\circ} \mathrm{C}$ in figure 6.7 and $100^{\circ} \mathrm{C}$ in figure 6.9 is compared. The 
highly steeped slope at $100^{\circ} \mathrm{C}$ is an indication of faster relaxation time. This can be due to the fact that the electrons are in excited state as a result of the gained heat energy or that reaction with oxygen occurs faster at high temperature.

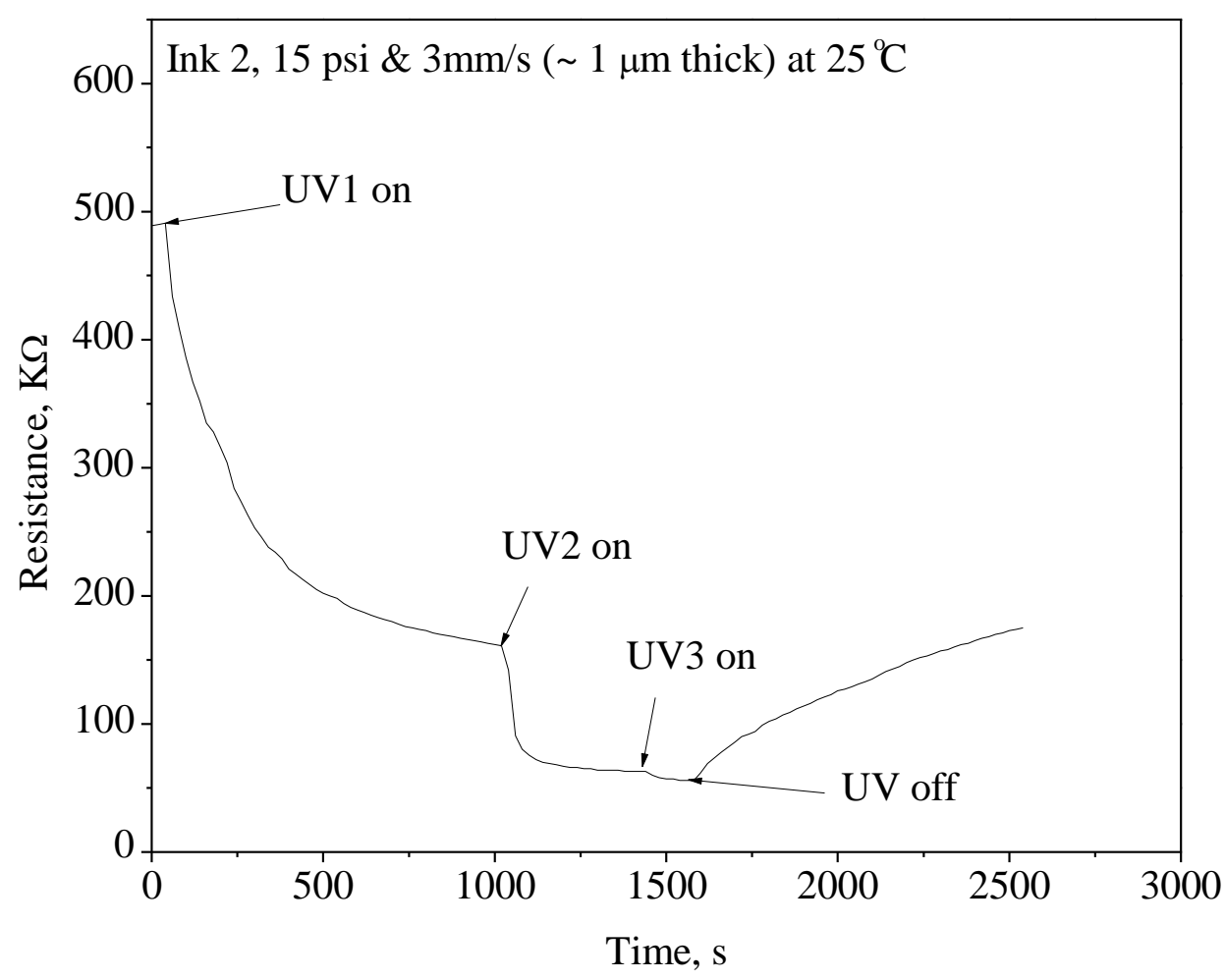

Figure 6.7 Transient resistance of AZO film deposited with ink 2, 15 psi and $3 \mathrm{~mm} / \mathrm{s}$ at $25^{\circ} \mathrm{C}$ 


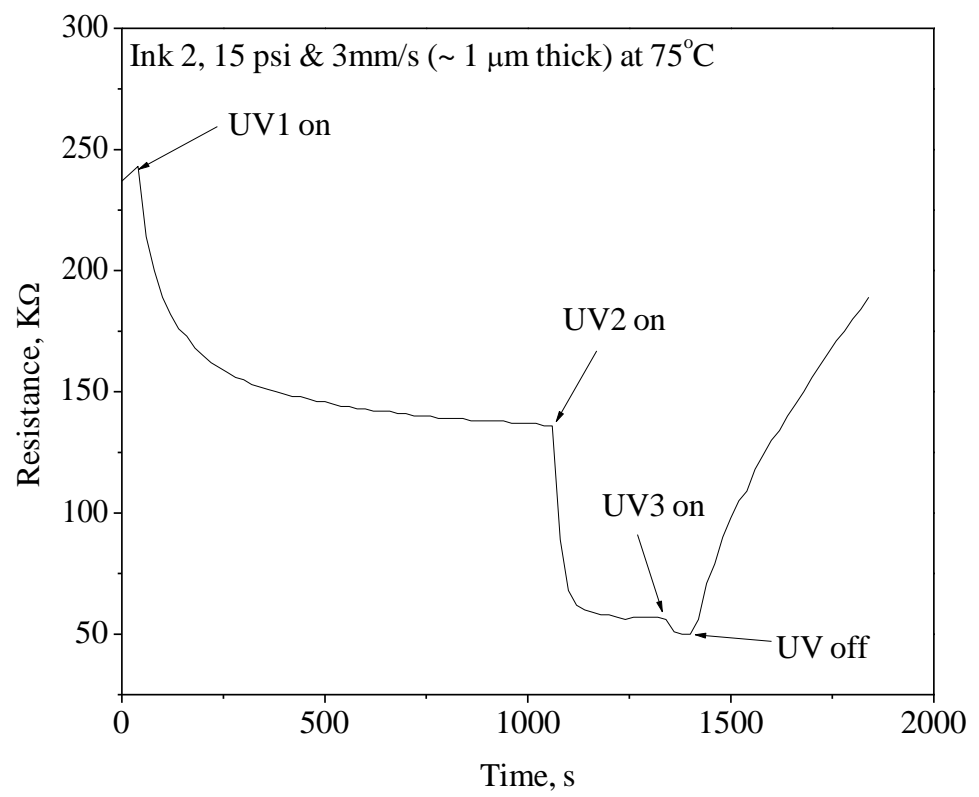

Figure 6.8 Transient resistance of AZO film deposited with ink 2, 15 psi and $3 \mathrm{~mm} / \mathrm{s}$ at $7^{\circ} \mathrm{C}$

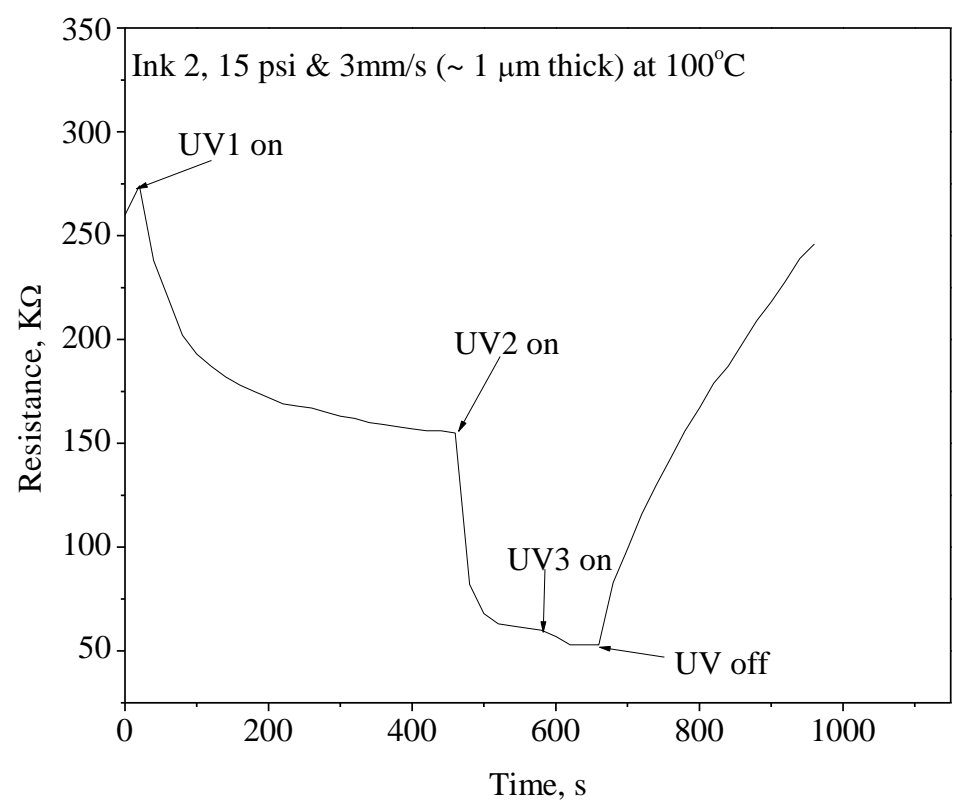

Figure 6.9 Transient resistance of AZO film deposited with ink 2, 15 psi and $3 \mathrm{~mm} / \mathrm{s}$ at $100^{\circ} \mathrm{C}$ 


\section{CONCLUSIONS AND RECOMMENDATIONS FOR FUTURE WORK}

In this work, we have printed thin film patterns of AZO synthesized by sol-gel method, on glass substrate through direct writing-NBRD technique. We studied the effects of the materials and printing parameters on the properties of the printed features. Viscosity was found to be of high importance in the printing operation as it affects the printing process right from ink extrusion to deposition. The effect of viscosity on the deposited film became more pronounced, while using larger tip size. The printing parameters; tip size, dispensing height, writing speed and extrusion pressure also affected the spread and consequently the width and profile of the printed AZO films. The spread increased with increase in tip size and extrusion pressure and conversely, decreased with increase in writing speed. The main effects, the two-factor interactions and the three-factor interactions of the printing parameters were all found to be significant in determining the width and geometry of the deposited AZO features, at $95 \%$ confidence level. Viscosity was found to be the most important factor, followed by tip size, writing speed and extrusion pressure, in that order. Writing speed and pressure were however, considered as the most important factor to optimize, due to their dynamic nature.

Heat treatment of the deposited film was found to entail drying of solvents in the film, decomposition of the polymer and crystallization of the $\mathrm{ZnO}$ to become functional. Significant physical geometry changes, as a result of sintering, were only noticed in the height of the deposited feature. Only slight differences were observed in the widths of as deposited and sintered patterns. The marked reduction in feature heights, compared to those of the widths of sintered patterns resulted in lower aspect ratios. 
The printing conditions also affected the morphology and crystal structure of the deposited films. Parameter combinations that resulted in deposition of larger volumes of materials caused formation of highly dense materials with smaller grain sizes, after sintering. On the other hand, less dense films were produced when smaller materials were deposited. The deposited films exhibit wurtzite structure with majorly c-axis oriented crystallites. The crystal formation and growth directions of the films' were also found to be influenced by the combinations of printing parameters and factors intrinsic to the materials. Features with widths less than $200 \mu \mathrm{m}$ were typically found to have crystal growth in the (002) plane, while those with widths between 200 and $400 \mu \mathrm{m}$ showed growth in 100, 002, and 101 directions, with a dominant 002 peak. Those greater than $400 \mu \mathrm{m}$ showed crystal growth in almost all of the growth directions found in $\mathrm{ZnO}$ wurtzite microstructure, with the 002 not necessarily being the dominant peak.

The I-V measurements showed ohmic relationship both with and without UV irradiation. The conductivity of the printed films were also affected by the printing conditions, as resistance to the flow of current differs for parameter combination. The resistance of the films were found to decrease upon irradiation with $365 \mathrm{~nm}$ UV for all film temperatures. The transient photo-response of the printed film showed that, excitation experienced upon exposure to UV is relaxed when the UV light is switched off. The relaxation time becomes shorter as film temperature is increased.

Future work in this direction would involve detailed investigation into the printing parameters and crystal-crystal interactions that led to the crystal growth in the chosen planes. Understanding of this may explain the dynamics of the crystal formation at certain widths, as earlier observed. Lower resolution printing, in the range lower than $20 \mu \mathrm{m}$, should be studied further, in order to check the validity of the trend previously observed. Studying of the surface chemistry of the sintered materials with the intention of establishing a relationship between it and 
its conductivity is also essential. Also, possible use of the deposited material in UV photo-detection devices should be considered. 


\section{RFERENCES}

[1] J. L. Gomez and O. Tigli, "Zinc oxide nanostructures: from growth to application,” Journal of Material Science, vol. 48, pp. 612-624, 2013.

[2] A. George, P. Kumari, N. Soin, S.S. Roy, J.A. McLaughlin, "Microstructure and field emission characteristics of $\mathrm{ZnO}$ nanoneedles grown by physical vapor deposition," Materials Chemistry and Physics, vo1. 23, pp. 634-638, 2010.

[3] N. U. Sangari, and S. C. Devi, "Synthesis and characterization of nano ZnO rods via microwave assisted chemical precipitation method," Journal of Solid State Chemistry, vol. 197, pp. 483488, 2013.

[4] X. Ye, Y. Zhou, J. Chen, Y. Sun, Z. Wang, "Coating of $\mathrm{ZnO}$ nanorods with nanosized silver particles by electroless plating," Materials Letters, vol. 62, pp. 666-669, 2008.

[5] Z. Szabo, J. Volk, E. Fulop, A. Deak, I. Barsony, "Regular ZnO nanopillar arrays by nanosphere photolithography," Photonics Nanostructures - Fundamentals and Applications, vol. 11, pp. 1-7, 2013.

[6] Y. Aoun, B. Benhaoua, S. Benramache, and B. Gasmi, "Effect of deposition rate on the structural, optical and electrical properties of Zinc oxide $(\mathrm{ZnO})$ thin films prepared by spray pyrolysis technique," Optik, Volume 126, no. 20, pp. 2481-2484, 2015.

[7] P. Prepelita, R. Medianu, F. Garoi, N. Stefan, F. Iacomi, "On the structural and electrical characteristics of zinc oxide thin films," Thin Solid Films, vol. 518, pp. 4615-4618, 2010.

[8] Z. Ng, K. Chan, C. Low, S. A. Kamaruddin and M. Z. Sahdan, "Al and Ga doped ZnO films prepared by a sol-gel spin coating technique," Ceramics International, vol. 41, sup. 1, pp. 254-258, 2015. 
[9] X. Wang, G. Zheng, G. He, J. Wei, H. Liu, Y. Lin, J. Zheng, D. Sun, “Electrohydrodynamic direct-writing ZnO nanofibers for device applications," Materials Letters, vol. 109, pp. 58-61, 2013.

[10] S.D. Cronin, K. Sabolsky, E.M. Sabolsky, K.A. Sierros, "Dip pen nanolithography and transfer of $\mathrm{ZnO}$ patterns on plastics for large-area flexible optoelectronic applications," Thin Solid Films, vol. 552, pp. 50-53, 2014.

[11] J. Y. Zheng, H. Y. Liu, X. Wang, Y. Zhao, W. W. Huang, G. F Zheng and D. H. Sun, "Electrohydrodynamic direct-write orderly micro/nanofibrous structure on flexible insulating substrate,” Journal of Nanomaterials, vol. 2014, pp. 708186 - 1-6, 2014.

[12] J. A. Lewis "Direct Ink Writing of 3D Functional Materials - Direct-write assembly of microperiodic planar and spanning ITO microelectrodes," Advanced Functional Materials, vol. 16, pp. 2193-2220, 2006.

[13] K. K. B. Hon, L. Li and I. M. Hutchings, "Direct writing technology-Advances and development," CIRP Annals - Manufacturing Technology, vol. 57, pp. 601-620, 2008.

[14] Guo, M. Heuzey, D. Theriault, "Properties of polylactide inks for solvent-cast printing of three-dimensional freeform microstructure," Langmuir, vol. 30, pp. 1142-1150, 2014.

[15] B. Y. Ahn, D. J. Lorang, E. B. Duoss and J. A. Lewis, "Direct-write assembly of microperiodic planar and spanning ITO microelectrodes," Chemical Communications, vol. 46, pp. 71187120, 2010.

[16] B. Y. Ahn, E. B. Duoss, M. J. Motala, X. Guo, S. Park, Y. Xiong, J. Yoon, G. Nuzzo, J. A. Rogers and J. A. Lewis, “Omnidirectional Printing of Flexible, Stretchable, and Spanning Silver Microelectrodes," Science, vol. 323, pp. 1590-1593 2009. 
[17] A. Pique and D. B. Chrisey, "Direct-Write Technologies for Rapid Prototyping Applications: Sensors,” Electronics and Integrated Power Sources. Academic Press, New York, 2002.

[18] J. A. Lewis and G. M. Gratson, "Direct Writing in Three Dimensions," Materials Today, vol. 7, no (7-8), pp. 32-39, 2004.

[19] PA Consultancy, "Direct Writing. Global Status and Opportunities for the UK in Advanced Manufacturing,” DTI, London, 2004.

[20] S. Z. Guo, F. Gosselin, N. Guerin, A. M. Lanouette, M. C. Heuzey and Daniel Therriault, "Solvent-cast three-dimensional printing of multifunctional microsystems," Small, vol. 9, no. 24, pp. 4118-4122, 2013.

[21] K. H. Church, C. Fore and T. Feeley, "Commercial Applications and Review for Direct Write Technologies,” MRS Proceedings, vol. 624, 3, 2011.

[22] J. A. Lewis, "Novel Inks for Direct-Write Assembly of 3-D Periodic Structures," Material Matters, vol. 3, no. 1, pp. 4-7, 2008.

[23] D. S. Engstrom, B. Porter, M. Pacios, H. Bhaskaran, "Additive nanomanufacturing - A review,” Journal of Materials Research, vol. 29, no. 17, pp. 1-25, 2014.

[24] L. Mortara, J. Hughes, P. S. Ramsundar, Finbarr Livesey David R. Probert, "Proposed classification scheme for direct writing technologies," Rapid Prototyping Journal, vol. 15 no. 4 pp. $299-309,2009$.

[25] M. Vaezi, H. Seitz and S. Yang, "A review on 3D micro-additive manufacturing technologies," International Journal of Advanced Manufacturing Technology, vol. 67, pp. 1721-1754, 2013. 
[26] Z. Pan, Y. Wang, H. Huang, Z. Ling, Y Dai, S Ke, "Recent development on preparation of ceramic inks in ink-jet printing," Ceramics International, vol. 41, no. 10, part A, pp. 12515$12528,2015$.

[27] J. M. Hoey, A .Lutfurakhmanov, D. L. Schulz and I. S. Akhatov, “A review on aerosol-based direct-rite and its applications for microelectronics” Journal of Nano technology, vol. 2012, pp. $1-22,2012$

[28] A. Selimis, V. Mironov and M. Farsari, "Direct laser writing: Principles and materials for scaffold 3D printing," Microelectronic Engineering, vol. 132, pp. 83-89, 2015.

[29] C. Kim, S. Ahn and D. Jang, "Review: Developments in micro/nanoscale fabrication by focused ion beams," Vacuum, vol. 86, no. 8, pp. 1014-103529, 2012.

[30] S. Eqtesadi, A. Motealleh, P. Miranda, A. Lemos, A. Rebelo and J. M. F. Ferreira, "A simple recipe for direct writing complex 45S5 Bioglasss 3D scaffolds," Materials Letters, vol. 93, pp. 68-71, 2013.

[31] J. C. Dingeldein, K. A. Walczak, B. W. Swatowski, C. R. Friedrich,C. T. Middlebrook and M. C. Roggemann, "Process characterization for direct dispense fabrication of polymer optical multi-mode waveguides," Journal of Micromechanics and Microengineering, vol. 23, no. 7, 075015, 2013.

[32] Y Noh, N .Zhao, M. Caironi and H. Sirringhaus, "Downscaling of self-aligned, all-printed polymer thin-film transistors," nature nanotechnology, vol. 2, pp.784-789, 2007.

[33] S. G. Bucella ,G. Nava, K. C. Vishunubhatla and M. Caironi, "High-resolution direct-writing of metallic electrodes on flexible substrates for high performance organic field effect transistors," Organic Electronics, vol. 14, no. 9, pp. 2249-2256, 2013. 
[34] J. K. Lee, U. J. Lee, M. Kim, S. H. Lee and K. Kang "Direct writing of semiconducting polythiophene and fullerene derivatives composite from bulk heterojunction solar cell by inkjet printing,” Thin Solid Films, vol. 519, no 16, pp. 5649-5653, 2011.

[35] K. Sun, T. S. Wei, B. Y. Ahn, J. Y. Seo, S. J. Dillon, and J. A. Lewis, “3D printing of interdigitated Li-Ion microbattery architectures," Advanced Materials, vol. 25, 4539-4543, 2013.

[36] Y. L. Kong, I. A. Tamargo, H. Kim, B. N. Johnson, M. K. Gupta, T. W. Koh, H. A. Chin, D. A. Steingart, B. P. Rand and M. C. McApine, "3D Printed Quantum Dot Light-Emitting Diodes," Nano Letters, vol. 14, 7017-7023, 2014.

[37] J. Bruneaux, D. Therriault and M. Heuzey, "Micro-extrusion of organic inks for directwrite assembly," Journal of Micromechanics and Microengineering, vo. 18, 115020, 2008.

[38] H. Morkoc and U. Ozgur, “General Properties of ZnO," Zinc Oxide: Fundamentals, Materials and Device Technology, WILEY-VCH Verlag GmbH \& Co. KGaA, Weinheim, 2009.

[39] K. L. Foo, U. Hashim, K. Muhammad and C. H. Voon, "Sol- gel synthesized zinc oxide nanorods and their structural and optical investigation for optoelectronic application," Nanoscale Research Letters, vol. 9, pp. 429-438, 2014.

[40] M. Xin, L. Z. Hu, D Liu and N Yu, "Effect of Mn doping on the optical, structural and photoluminescence properties of nanostructured $\mathrm{ZnO}$ thin film synthesized by sol-gel technique," Superlattices and Microstructures, vol.74, pp. 234-241, 2014.

[41] H. Li, L. K. Schirra, J. Shim, H. Cheun, B. Kippelen, O. L. A. Monti, and J. Bredas, “Zinc oxide as a model transparent conducting oxide: a theoretical and experimental study of the impact of hydroxylation, Vacancies, Interstitials, and Extrinsic Doping on the Electronic 
Properties of the Polar ZnO (0002) Surface," Chemistry of Materials, vol. 24, pp. 3044-3055, 2012.

[42] K. S. Babu , A. R. Reddy, C. Sujatha and K. V. Reddy, "Effects of precursor, temperature, surface area and excitation wavelength on photoluminescence of $\mathrm{ZnO} /$ mesoporous silica nanocomposite," Ceramics International, vol. 39, pp.3055 - 3064, 2013.

[43] J. Singh, P. Kumar, K. S. Hui, K. N. Hui, K. Ramam, R. S. Tiwari and O. N. Srivastava, "Synthesis, band-gap tuning, structural and optical investigations of Mg doped ZnO nanowires,” Crystal Engineering Communications, vol. 14, pp. 5898-5904, 2012.

[44] K. S. Babu, A. R. Reddy, C. Sujatha, K.V.G. Reddy and A. N. Mallika, "Annealing effects on photoluminescence of ZnO nanoparticles," Materials Letters ,vol.110, pp.10-12, 2013.

[45] Z. Fan and J. G. Lu, "Zinc oxide nanostructures: synthesis and properties," Journal of Nanoscience and Nanotechnology, vol. 5, no. 10, pp. 1561-1573, 2005.

[46] Y. Jiang, W. Wang, C. Jing, C. Cao, J. Chu, "Sol-gel synthesis, structure and magnetic properties of Mn-doped $\mathrm{ZnO}$ diluted magnetic semiconductors," Materials Science and Engineering: B, vol. 176, no. 16, pp. 1301-1306, 2011.

[47] L. Znaidi, "Sol-gel-deposited ZnO thin films: A review," Materials Science and Engineering B, vol. 174, pp. 18-30, 2010.

[48] S. Khodja, T. Touam, A. Chelouche, F. Boudjouan, D. Djouadi, Z. Hadjoub, A. Fischer and A. Boudrioua, "Effects of stabilizer ratio on structural, morphological, optical and waveguide properties of $\mathrm{ZnO}$ nano-structured thin films by a sol-gel process," Superlattices and Microstructures, vol. 75, pp. 485-495, 2014.

[49] U. Choppali, E. Kougianos, S. P. Mohanty and B. P. Gorman, "Maskless deposition of ZnO films," Solar Energy Materials \& Solar Cells, vol. 95, pp. 870-876, 2011. 
[50] A. Gahtar, A. Rahal , B. Benhaoua and S. Benramache," A comparative study on structural and optical properties of $\mathrm{ZnO}$ and $\mathrm{Al}$-doped $\mathrm{ZnO}$ thin films obtained by ultrasonic spray method using different solvents,” Optik, vol. 125, pp. 3674-3678, 2014.

[51] S. O'Brien, M. Çopuroglu, P. Tassie, M. G. Nolan, J. A. Hamilton, I. Povey, L. Pereira, R. Martins, E. Fortunato, M. E. Pemble, "The effect of dopants on the morphology, microstructure and electrical properties of transparent zinc oxide films prepared by the solgel method," Thin Solid Films, vol. 520, pp.1174-1177, 2011.

[52] M. Gao , X. Wu , J. Liu and W. Liu ,"The effect of heating rate on the structural and electrical properties of sol-gel derived Al-doped ZnO films,” Applied Surface Science, vol. 257, pp. 6919-6922, 2011.

[53] R. Mimouni, O. Kamoun, A. Yumak, A. Mhamdi, K. Boubaker, P. Petkova M. Amlouk "Effect of Mn content on structural, optical, opto-thermal and electrical properties of $\mathrm{ZnO}: \mathrm{Mn}$ sprayed thin films compounds," Journal of Alloys and Compounds, vol. 645, pp.100-111, 2015.

[54] S. M. Rozati, S. Moradi, S. Golshahi, R. Martins, E. Fortunato, "Electrical, structural and optical properties of fluorine-doped zinc oxide thin films: Effect of the solution aging time." Thin Solid Films, vol. 518, pp. 1279-1282, 2009.

[55] P. S. Shewale, N. K. Lee, S. H. Lee, K.Y. Kang, Y. S. Yu, “Ti doped ZnO thin film based UV photodetector: fabrication and characterization" Journal of Alloys and Compounds, vol. 624, pp. 251-257, 2015.

[56] W. S. Khan, C. Cao, G. Nabi, R. Yao and S. H. Bhatti ," Catalyst-free combined synthesis of $\mathrm{Zn} / \mathrm{ZnO}$ core/shell hollow microspheres and metallic $\mathrm{Zn}$ micro particles by thermal 
evaporation and condensation route," Journal of Alloys and Compounds, vol. 506, no. 2, pp. 666-672, 2010.

[57] S. Fay, U. Kroll, C. Butcher, E. Vallat-Sauvain and A Shah, "Low pressure chemical vapour deposition of $\mathrm{ZnO}$ layers for thin-film solar cells: temperature-induced morphological changes," Solar Energy Materials and Solar Cells, vol. 86, no. 3, pp. 385-397, 2005.

[58] K. Maejima, T. Koida, H. Sai, T. Matsui, K. Saito, M. Kondo and T. Takagawa, "Influences of deposition temperature on characteristics of B-doped $\mathrm{ZnO}$ films deposited by metalorganic chemical vapor deposition,” Thin Solid Films, vol. 559, pp. 83-87, 2014.

[59] N. A. Ahmed, H. Hammache, L. Makhloufi, M. Eyraud, S. Sam, A. Keffous and N. Gabouze, "Effect of electrodeposition duration on the morphological and structural modification of the flower-like nanostructured ZnO," Vacuum, vol. 120, B, pp.100-106, 2015.

[60], L. Chang, K. H. Ploog and M. M. C. Chou, "Epitaxial growth of nonpolar ZnO on MgO (100) substrate by molecular beam epitaxy," Journal of Crystal Growth, vol. 378, pp. 172$176,2013$.

[61] M. S. Al-Assiri, M. M. Mostafa, M. A. Ali and M. M. El-Desoky, "Synthesis, structural and electrical properties of annealed $\mathrm{ZnO}$ thin films deposited by pulsed laser deposition (PLD),"Superlattices and Microstructures, vol. 75, pp. 127-135, 2014.

[62] L. F. Koao, F. B. Dejene and H. C. Swart, "Properties of flower-like ZnO nanostructures synthesized using the chemical bath deposition," Materials Science in Semiconductor Processing vol. 27, pp. 33-40, 2014.

[63] J.R. Casanova, E.A. Heredia, C.D. Bojorge, H. R. Cánepa, G. Kellermann, A. F. Craievich, "Structural characterization of supported nanocrystalline $\mathrm{ZnO}$ thin films prepared by dipcoating,” Applied Surface Science, vol. 257, no 23, 15, pp. 10045-10051, 2011. 
[64] K. L. Foo, M. Kashif, U. Hashim and W. Liu, "Effect of different solvents on the structural and optical properties of zinc oxide thin films for optoelectronic applications," Ceramics International, vol.40, pp.753-761, 2014.

[65] A. Axelevitch, B. Gorenstein, H. Darawshe and G. Golan, "Investigation of thin solid ZnO films prepared by sputtering,” Thin Solid Films, vol. 518, pp. 4520-4524, 2010.

[66] A. T. Vai, V. L. Kuznetsov, J.R. Dilworth and P. P. Edwards "UV-induced improvement in ZnO thin film conductivity: a new in situ approach," Journal of Materials Chemistry, C, vol. 2, pp. 9643-9652, 2014.

[67] T. H Wu, I. C. Cheng, C. C. Hsu and J. Z. Chen, "UV photocurrent responses of ZnO and $\mathrm{MgZnO} / \mathrm{ZnO}$ processed by atmospheric pressure plasma jets," Journal of Alloys and Compounds, vol. 628, pp. 68-74, 2015.

[68] A. Mahroug, S. Boudjadar, S.Hamrit, L. Guerbous, "Structural, optical and photocurrent properties of undoped and Al-doped $\mathrm{ZnO}$ thin fi lms deposited by sol- gel spin coating technique," Materials Letters, vol. 134, pp. 248-251, 2014.

[69] A. Bera and D. Basak, Photoluminescence and Photoconductivity of $\mathrm{ZnS}$-Coated $\mathrm{ZnO}$ Nanowires,” Applied Materials and Interfaces, vol. 2, no. 2, pp. 408-412, 2010.

[70] J. Bao , I. Shalish, Z. Su , R. Gurwitz, F. Capasso, X. Wang and Z. Ren, ”Photoinduced oxygen release and persistent photoconductivity in $\mathrm{ZnO}$ nanowires," Nanoscale Research Letters, vol. 6, pp. 404-410, 2011.

[71] S. Hullavarad, N. Hullavarad, D. Look and Bruce Claflin, "Persistent Photoconductivity Studies in Nanostructured ZnO UV Sensors," Nanoscale Research Lettesr, vol. 4, pp. 142114272009. 
[72] A. P. Tafti, A. B. Kirkpatrick, Z. Alavi, H. A. Owen, and Z. Yu, "Recent advances in 3D SEM surface reconstruction,” Micron, vol. 78, pp. 54-66, 2015.

[73] T. X. Liang, W. Z. Sun, L. D. Wang, Y. H. Wang, and H. D. Li, "Effect of surface energies on screen printing resolution," IEEE Transactions on Components, Packaging, and Manufacturing Technology-Part B, vol. 19, no. 2, pp. 423- 426, 1996.

[74] H. A. D Nguyen, J. Lee, C. H. Kim, K. Shin and D. Lee,” An approach for controlling printed line-width in high resolution roll-to-roll gravure printing" Journal of Micromechanics and . Microengineering, vol. 23, 095010, 2013.

[75] J. W. Boley, E. L. White, G. T. Chiu and R. K. Kramer, "Direct Writing of gallium-indium alloy for stretchable electronics, “Advance Functional Materials, vol. 24, pp. 3501-3507, 2014.

[76] J. Yang, V. Fridrici, M. Messaadi, Ph. Kapsa, "Survival and factorial analysis of durability and friction coefficient of a solid lubricant under different working conditions," Wear, vol. 302, pp. 998-1009, 2013.

[77] G. Vozzi, A. Previti, D. De Rossi, A. Ahluwalia, "Microsyringe-based deposition of twodimensional and three-dimensional polymer scaffolds with a well-defined geometry for application to tissue engineering," Tissue Engineering, vol. 8, no. 6, pp. 1089-1098, 2002.

[78] N. Fujimura, T. Nishihara, S. Goto, J. Xu and T. Ito, "Control of preferred orientation for $\mathrm{ZnO}_{\mathrm{x}}$ films: control of self texture," Journal of Crystal Growth, vol. 130, no. 1-2, pp. 269$279,1993$.

[79] T. Ates, C. Tatar and F. Yakuphanoglu, "Preparation of semiconductor $\mathrm{ZnO}$ powders by solgel method: Humidity sensors," Sensors and Actuators A, vol. 190, pp. 153-160, 2013. 
[80] M. Dhingra, N. K. Singh, S. Shrivastava, P. S. Kumar S. Annapoorn,” Worm like zinc oxide nanostructures as efficient LPG sensors," Sensors and Actuators A, vol. 190, pp. 168-175, 2013.

[81] M. R. Islam and J. Podder. "Optical properties of $\mathrm{ZnO}$ nano fiber thin films grown by spray pyrolysis of zinc acetate precursor, "Crystal Research and Technology, vol. 44, no. 3, pp. 286292, 2009.

[82] S.C. Su, Y.M. Lu, Z.Z. Zhang, B.H. Li , D.Z. Shen, B. Yao , J.Y. Zhang, D.X. Zhao and X.W. Fan, "Structural, optical, and hydrogenation properties of $\mathrm{ZnO}$ nanowall networks grown on a Si (111) substrate by plasma-assisted molecular beam epitaxy," Physica B, vol. 403 pp. 2590-2593, 2008.

[83] Y. Natsume and H. Sakata, "Zinc oxide films prepared by sol-gel spin-coating" Thin Solid Films, vol. 372, pp. 30-36, 2000.

[84] J. Wang, Y. Qi, Z. Zhi, J. Guo1, M. Li1 and Y. Zhang, 'A self-assembly mechanism for solgel derived ZnO thin films," Smart Material Structure, vol. 16, pp. 2673-2679, 2007.

[85] Y. Z. Zhu, G. D. Chen, and H. Ye, "Electronic structure and phase stability of MgO, ZnO, CdO, and related ternary alloys," Physical Review B, vol. 77, 245209, 2008. 